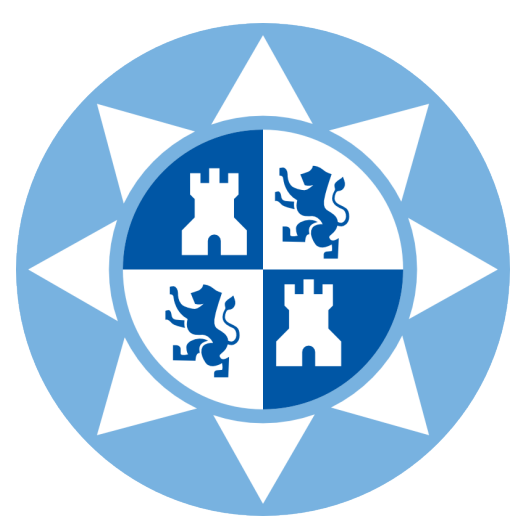

Universidad Politécnica de Cartagena

Departamento de Tecnologías de la Información y las Comunicaciones

\title{
Planificación y Optimización de Redes Ópticas en el Internet del Futuro
}

Francisco Javier Moreno Muro

\author{
Directores \\ Pablo Pavón Mariño \\ María Victoria Bueno Delgado
}





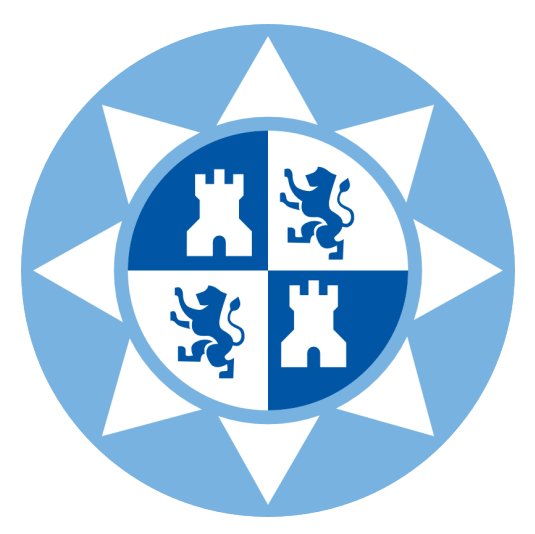

Technical University of Cartagena

Department of Information Technologies and Communications

\title{
Planning and Optimization in Optical Network for Future Internet
}

Francisco Javier Moreno Muro

\author{
Supervisors \\ Pablo Pavón Mariño \\ María Victoria Bueno Delgado
}

2019 

A los míos. 



\section{Abstract}

The strict requirements required for the future $5 \mathrm{G}$ network, jointly with the high growth forecasts of IP traffic, mainly based on the proliferation of cloud services, create a future panorama full of uncertainties from the perspective of large optical telecommunications networks. Planning and optimization tasks are essential to ensure that the requirements are satisfied in an economically viable manner.

This thesis tries to analyze, in the first place, the validity of traffic growth predictions, in the light of current applications in a scenario where the latency requirements imply the evolution of CDNs with the deployment of micro-datacenters. In particular, the effect of this traffic evolution, supported by the optical backbone networks, will be analyzed. Second, the thesis studies some options that allow addressing the expected requirements of the network, from the perspective of three major approaches: a) optimization in the design and management of CDNs, b) programmable control of the network based on software-defined networking and with virtualization in network functions and finally, c) possible introduction of the Space Division Multiplexing (SDM) technology to expand the capacity of transport networks as effective support for the increase of IP traffic.

The outcomes of this work conclude, in the first instance, that the evolution of the backbone traffic in the future Internet will depend on the nature of the services that the applications offer, being the balance between user traffic and synchronization one especially determining. On the other hand, the results suggest that place the content close to the users can have direct effect of decreasing the amount of traffic supported by the core networks, in relation to the current forecasts.

The use cases analyzed in the SDN-NFV environment determine the need for optimization providing flexibility and programmability in the migration to virtualized systems in the networks. This set of functionalities are essential to satisfy the requirements of the future services in the 5G paradigm. In addition, the proof of concept presented in this thesis supports the joint optimization of network and IT resources for the assistance of service chains in networks based on SDN-NFV.

Finally, for SDM-based optical networks, the proposal presented for space channel restriction (SCC) emerges as a valid option to be implemented in ROADMs for flex-grid environments. The validity of this proposal lies in the significant reduction in terms of implementation cost due to its lower complexity and the current availability of the equipment, at the expense of minimal performance degradations, compared to totally permissive options. 



\section{Resumen}

Las estrictas exigencias requeridas por la futura red $5 \mathrm{G}$, junto a las elevadas previsiones de crecimiento de tráfico IP, principalmente cimentadas en la proliferación de numerosos servicios basados en la nube, crean un panorama futuro lleno de incógnitas desde la perspectiva de las grandes redes ópticas de telecomunicaciones. Las tareas de planificación y optimización serán esenciales para asegurar que los requisitos pueden cumplirse de una manera económicamente viable.

Esta tesis trata de analizar, en primer lugar, la validez de las predicciones de crecimiento tráfico, a la luz de las actuales aplicaciones y en un escenario donde los requisitos de latencia implican la evolución de los CDNs con el despliegue de micro-datacenters. Se analizará en particular el efecto de esta evolución en el tráfico soportado por las redes ópticas troncales. Segundo, la tesis estudia opciones que permitan abordar los requisitos esperados de la red, desde tres enfoques: a) optimización en el diseño y gestión de CDNs, b) control programable de la red basado, en redes definidas por software (SDN) y con virtualización en las funciones de red (NFV) y por último, c) posible introducción de tecnología SDM (Space Division Multiplexing) para expandir la capacidad de las redes de transporte como soporte efectivo del incremento de tráfico IP.

Los resultados de este trabajo concluyen, en primera instancia, que la evolución del tráfico troncal en el Internet del futuro dependerá de la naturaleza de los servicios que las aplicaciones ofrezca, siendo el balance entre el tráfico de usuario y de sincronización especialmente determinante. Por otro lado, los resultados sugieren que un acercamiento del contenido a los usuarios puede tener el efecto neto de disminuir la cantidad de tráfico soportada por las redes core, en relación con las previsiones actuales.

Los casos de uso analizados en el entorno SDN-NFV determinan la necesidad de optimización para proporcionar flexibilidad y programabilidad en la migración hacia sistemas virtualizados en las redes, siendo este conjunto de funcionalidades esenciales para satisfacer los requerimientos de los futuros servicios en el paradigma 5G. Además, las pruebas de concepto presentadas avalan la optimización conjunta de recursos de red e IT para la asistencia de service chains en redes basadas en SDN-NFV.

Finalmente, para las redes ópticas basadas en SDM, la propuesta presentada de restricción de canal espacial (SCC) emerge como una opción válida a implementar en ROADMs para contextos flex-grid. La validez de tal propuesta reside en la reducción de coste de implementación dada por su menor complejidad y mayor disponibilidad de equipamiento, a costa de mínimas pérdidas en rendimiento, respecto de opciones totalmente permisivas. 



\section{Agradecimientos}

Posiblemente, lo mas complicado de la escritura de esta tesis sea realizar los agradecimientos en la justa medida que cada una de las personas implicadas merecen. Hay tanta gente, y a tantos niveles, que han puesto su aporte para que sea posible este trabajo que sería imposible hacer honor a la realidad en un conjunto finito de palabras. A todos ellos, mi más sentido GRACIAS. Por este motivo, siguiendo el principio de la propiedad conmutativa, el orden de los agradecimientos expuestos a continuación no implica distinta relevancia.

En primer lugar, me gustaría tomarme la libertad de comenzar agradeciendo especialmente a Pablo y Victoria la oportunidad que en su día me dieron para poder formar parte, no sólo de este proyecto, sino de un grupo magnífico de trabajadores y, principalmente, personas. También agradecer la predisposición e integración que me han proporcionado a nivel personal y profesional a todo el grupo GIRTEL/ELI, con mención específica a César, Nina y Miquel, cuya aportación a esta tesis ha sido especialmente determinante.

Difícilmente hubiera llegado siquiera al principio de este viaje sin haber compartido gran parte de mi vida con mi enorme grupo de amigos. A Tito, Manu, Pony, Dani, Selu, Elena, Lourdes, Jose, Isa, Nonos, Pepe, Estela, Antonio y a todos los que han ayudado a formarme como persona, no se imaginan cuánto de ellos hay en este trabajo. ¡Muchísimas gracias!

La base de quién soy y seré, es y siempre será de mi(s) familia(s), tanto la de Úbeda como la de Puente Genil. No solo he recibido una magnífica educación y apoyo incondicional, sino también, en cierta medida, me he visto beneficiado por estar en contacto con cualidades extraordinarias. De mi padre, la importancia del conocimiento y la comprensión, que no hay objetivo mas importante que el de ser feliz. Mi madre, su increíble templanza y paciencia, dos de las cualidades mas importantes a considerar, no solo en un trabajo como el de investigador, además como forma de vida. Y finalmente, mi hermano, con su infinita capacidad de trabajo y constancia, posiblemente, una de las personas mas trabajadoras que conozco.

Y por supuesto...Miriam, mi presente y mi futuro, quién ocupa mi primer y último pensamiento cada día. Gracias vidi, por darme esa tranquilidad y apoyo que he necesitado tanto durante todo este tiempo.

A todos...de todo corazón, gracias. 



\section{Lista de Contentido}

1. Introducción 1

1.1. Introducción general . . . . . . . . . . . . . . . . . 1

1.2. Crecimiento del tráfico IP e impacto en las redes de transporte . . . . . . 3

1.3. Propuestas para afrontar el Internet del futuro . . . . . . . . . . . . 4

1.3.1. Descongestión de redes troncales, planificación de CDNs . . . . . 5

1.3.2. Optimización de recursos de red e IT, SDN-NFV . . . . . . . . 5

1.3.3. Expansión de redes ópticas, flex-grid y SDM . . . . . . . . . 6

1.4. Publicaciones derivadas de la tesis . . . . . . . . . . . . 6

1.5. Proyectos enmarcados en el desarrollo de la tesis . . . . . . . . . . 7

1.5.1. Proyecto ONOFRE . . . . . . . . . . . . . . . . 7

1.5.2. Proyecto ONOFRE-2 . . . . . . . . . . . . . . . . . . . . . . 8

1.5.3. Red de excelencia Elastic Networks . . . . . . . . . . . . . . 9

1.5.4. Metro-Haul H2020 Project . . . . . . . . . . . . . . . . 9

1.6. Estructura de la tesis . . . . . . . . . . . . . . . . 10

2. Estado y Evolución de Redes Troncales 11

2.1. Introducción y estado del arte . . . . . . . . . . . . . . . . . 12

2.2. Problemática e hipótesis inicial . . . . . . . . . . . . . . . . . . . . . . . 14

2.3. Modelo de evolución temporal de red . . . . . . . . . . . . 16

2.3.1. Política de crecimiento de CDN . . . . . . . . . . . . . . . 17

2.3.2. Modelado de tráfico IP. Servicios y aplicaciones . . . . . . . . . . 18

2.3.2.1. Tráfico User-to-user . . . . . . . . . . . . 18

2.3.2.2. Tráfico DC-to-user . . . . . . . . . . . 18

2.3.2.3. Tráfico $D C$-to-DC . . . . . . . . . . . . 20

2.3.3. Algoritmo de optimización para la evolución de tráfico core (MP-

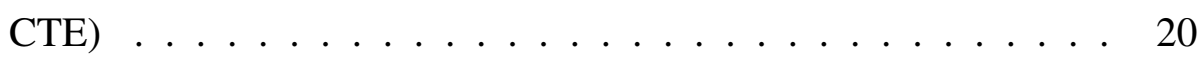

2.3.3.1. ILP para localización de réplicas (ILP-RP) . . . . . . . 21 
2.4. Resultados numéricos . . . . . . . . . . . . . . . . . . . . 24

2.4.1. Evolución de tráfico core bajo crecimiento de CDNs . . . . . . 24

2.4.1.1. Escenario de simulación . . . . . . . . . . . . . . 24

2.4.1.2. Resultados . . . . . . . . . . . . . . . 25

2.4.2. Análisis paramétrico de servicios . . . . . . . . . . . . 27

2.5. Conclusiones . . . . . . . . . . . . . . . . . . . 29

3. Gestión y Optimización de Redes SDN/NFV 31

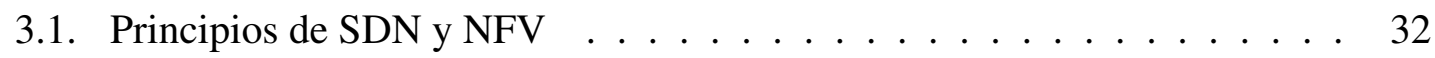

3.1.1. Arquitectura de SDN . . . . . . . . . . . . . . . . 32

$3.1 .2 . \quad$ Arquitectura de NFV . . . . . . . . . . . . . . . . 34

3.1.3. Combinación de SDN y NFV . . . . . . . . . . . . 35

3.2. Motivación de uso y herramientas open source . . . . . . . . . . 36

3.2.1. Controladores SDN . . . . . . . . . . . . . . . . . . 36

3.2.1.1. Open Networking Operating System (ONOS) _ . . . . 37

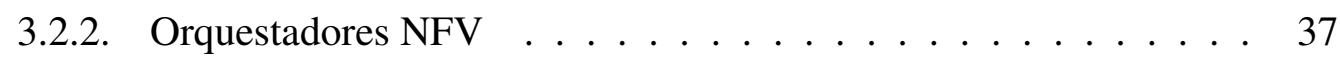

3.2.2.1. Open Source MANO (OSM) … . . . . . . . . 37

3.2.3. Gestores de infraestructuras virtuales, VIMs . . . . . . . . . . 38

$3.2 .3 .1 . \quad$ OpenStack . . . . . . . . . . . . . . . . . . . 39

3.3. Optimización en entornos SDN-NFV . . . . . . . . . . . . . . 39

3.3.1. Optimización en capa óptica de transporte. . . . . . . . . . . . . 39

3.3.1.1. Caso de uso: optimización de QoT . . . . . . . . . . 40

3.3.2. Optimización de redes de transporte IP sobre WDM . . . . . . 40

3.3.2.1. Caso de uso: optimización de recursos en redes SDN. . 41

3.3.3. Optimización en la asignación de recursos IT . . . . . . . . . 42

3.3.3.1. Caso de uso: optimización de recursos para VNFs . . . 42

3.3.4. Optimización conjunta para recursos de red e IT en redes de trans-

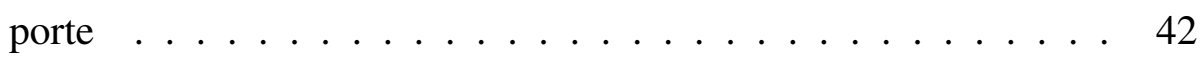

3.3.4.1. Caso de uso: optimización de service chain con múltiples VIMs conectados a una red de trasporte . . . . . . 43

3.4. Gestión en SDN-NFV. Pruebas de concepto . . . . . . . . . . . . . 44

3.4.1. Demostración de asignación conjunta de cadena de servicios, instanciación de VNFs y gestión de recursos en redes metro . . . . . 44

3.4.1.1. Esquema funcional de la demostración . . . . . . . . . 44

3.4.1.2. Implementación y configuración . . . . . . . . . 46

3.4.1.3. Flujo de ejecución y resultados . . . . . . . . . . 47

3.4.2. Optimización en service chain basada en latencia con creación de instancias VNFs y control SDN de red metro . . . . . . . . . . . 49 
3.4.2.1. Visión esquemática de la demostración . . . . . . . . . 49

3.4.2.2. Implementación y configuración . . . . . . . . . 51

3.4.2.3. Flujo de ejecución y resultados . . . . . . . . . . 51

3.5. Conclusiones y trabajos futuros . . . . . . . . . . . . . . . 57

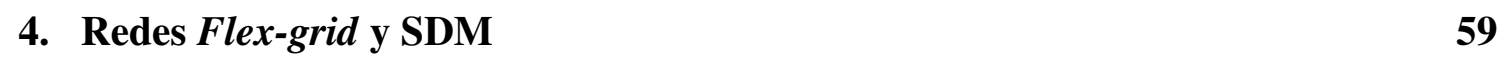

4.1. Aumento de la capacidad de red. Flex-grid y SDM . . . . . . . . . . 60

4.1.1. Redes elásticas . . . . . . . . . . . . . . . . 60

4.1.2. Multiplexación mediante división espacial . . . . . . . . . . . . . 61

4.1.2.1. Fibras con varios núcleos . . . . . . . . . . 61

4.1.2.2. Súper canales espaciales . . . . . . . . . . . 61

4.2. Estado del arte. . . . . . . . . . . . . . . . . . . 62

4.3. Arquitecturas de ROADM en contextos SDM y flex-grid . . . . . . . . . 64

4.3.1. Fully-Non-Blocking ROADM . . . . . . . . . . . . . 65

4.3.2. Joint-Switching ROADM . . . . . . . . . . . . . . . . 66

4.3.3. Spatial-Continuity-Constraint ROADM . . . . . . . . . . 66

4.3.4. Comparación de arquitecturas . . . . . . . . . . . . . . . 67

4.3.4.1. Broadcast-and-Select. FNB vs. CCC . . . . . . . . 67

4.3.4.2. Route-and-Select. FNB vs. JoS vs. SCC . . . . . . 69

4.4. Análisis de escenario estático . . . . . . . . . . . . . . . . . . . 70

4.4.1. Algoritmos . . . . . . . . . . . . . . 70

4.4.1.1. Algoritmo ILP . . . . . . . . . . . . . 70

4.4.1.2. Algoritmo Heurístico . . . . . . . . . . . . 72

4.4.2. Descripción del escenario estático y resultados . . . . . . . . . 73

4.4.2.1. Validación del heurístico . . . . . . . . . . 74

4.4.2.2. Análisis de rendimiento de CCC-ROADM . . . . . . 78

4.5. Análisis de escenario dinámico . . . . . . . . . . . . . . . . . 80

4.5.1. Algoritmo heurístico RMSSA . . . . . . . . . . . . . . . 80

4.5.2. Configuración del escenario dinámico . . . . . . . . . . . . . . . . . 81

4.5.3. Resultados y discusión . . . . . . . . . . . . . . 82

4.5.3.1. Estudio tecno-económico . . . . . . . . . 85

4.6. Conclusiones . . . . . . . . . . . . . . . . . . 86

\begin{tabular}{|lr}
\hline 5. Conclusiones y líneas futuras & 87
\end{tabular}

5.1. Conclusiones . . . . . . . . . . . . . . . . 87

5.2. Trabajos futuros . . . . . . . . . . . . . . . . 88 



\section{Lista de Figuras}

2.1. Predicciones de tráfico IP según [Cisco17] para distintos servicios y el total de tráfico IP. . . . . . . . . . . . . . . . . . 12

2.2. Ejemplo de expansión de CDN y reparto asociado de réplicas para (a) bajo

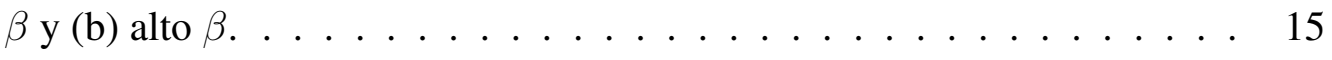

2.3. Modelado de popularidad de contenido: distribución normalizada de Zipf 1/f. . . . . . . . . . . . . . . . . . . 19

2.4. Algoritmo de optimización para la evolución de tráfico core (MP-CTE) . $\quad 22$

2.5. Tráfico total en los enlaces core para las topologías (a) NSFNet y (b) EON, durante 15 años. . . . . . . . . . . . . . . . . 26

2.6. (a) Tráfico total cursado en los enlaces core y (b) número total de DCs por - CDN para distintas aplicaciones. . . . . . . . . . . . 28

3.1. Arquitectura lógica de una red definida por software (SDN). Fuente: [OpenN12].

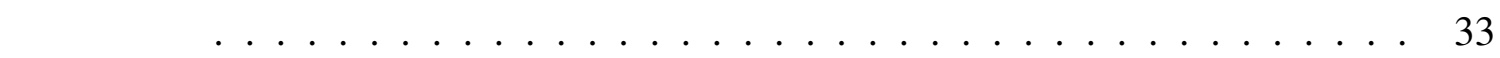

3.2. Arquitectura lógica de NFV. Fuente: [Mijum16]. . . . . . . . . . . . . . 34

3.3. Ejemplo de service chain en un entorno de combinación SDN-NFV. . . . 35

3.4. Estructura de bloques de OSM Release FOUR. Fuente [Osm]. . . . . . . 38

3.5. Caso de uso: optimización de QoT en redes SDN. Fuente: [Garri18a]. . . 41

3.6. Caso de uso: optimización de recursos en redes SDN. Fuente: [Garri18a].] 41

3.7. Caso de uso: optimización de recursos IT para, a) una VNF, b) varias VNFs. Fuente: [Garri18a]. . . . . . . . . . . . . . . . . . . . . 43

3.8. Caso de uso: optimización de service chain con múltiples VIMs conectados a una red de trasporte. Fuente: [Garri18a]. . . . . . . . . . . . . . . 44 44

3.9. Esquema funcional de la prueba de concepto presentada en [Moren18b]. . 45

3.10. Esquema de la implementación de la demostración [Moren18b]. . . . . . 46

3.11. Algoritmo para asignación de recursos de red e IT y localización de VNFs en $[$ Moren18b]. . . . . . . . . . . . . . . . . . . . . . . . . . 48 
3.12. Captura en la GUI de Net2plan tras ejecución de la demostración [Moren18b]. 49

3.13. Esquema funcional de la demostración [Moren18d]. . . . . . . . . . . . . 50

3.14. Diseño de la implementación de la demostración [Moren18d]. . . . . . . 52

3.15. Algoritmo para asignación de recursos de red e IT y localización de VNFs en [Moren18d]. . . . . . . . . . . . . . . . . . . . . . . . . 54

3.16. Captura de la interfaz gráfica de usuario en Net2plan. Demostración [Moren18d]. 55

3.17. Interfaz gráfica de OSM con VNFs instanciadas. Demostración [Moren18d]. 55

3.18. GUI de ONOS con la topología utilizada en [Moren18d] resaltando el camino seguido por el flujo. . . . . . . . . . . . . . . . . . 56

3.19. GUI de ONOS mostrando los flujos de control y datos establecidos en el

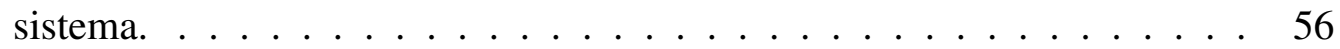

4.1. Uso espectral para tecnologías fixed-grid vs. flex-grid. Fuente [Imran18]. . 60

4.2. Tipos de asignación de SCh. (a) Spa-SCh, (b) S2-SCh y (c) Spe-SCh. Fuente: [Rumip18]. . . . . . . . . . . . . . . . . . 62

4.3. Arquitecturas de nodo óptico: (a) Broadcast-and-select y (b) Route-and-

select. Fuente: [Garri15]. . . . . . . . . . . . . . . . . . 63

4.4. Arquitectura de nodo óptico tipo R\&S Fully-Non-Blocking . . . . . . . . 65

4.5. Arquitectura de nodo óptico tipo R\&S Joint-Swithcing . . . . . . . . . . 66

4.6. Arquitectura de nodo óptico tipo Spatial-Continuity-Constraint . . . . . 67

4.7. Pseudocódigo del algortimo heurístico para resolver el RMSCA. . . . . . 73

4.8. Máximo tráfico cursado $v s$. FS ocupados para ILP en T7S . . . . . . . . 76

4.9. Máximo tráfico cursado $v s$. FS ocupados para heurístico en T7S . . . . . 76

4.10. Máximo tráfico cursado $v s$. FS ocupados para ILP en I2 . . . . . . . . . . . 77

4.11. Máximo tráfico cursado $v s$. FS ocupados para heurístico en I2 . . . . . . 77

4.12. Máximo tráfico llevado según el heurístico para diferentes topologías y

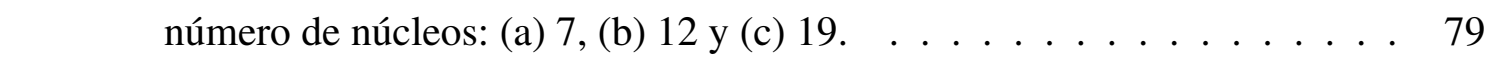

4.13. Pseudocódigo del algortimo heurístico para resolver el RMSSA. Fuente: [Rumip18]...................... 81

4.14. Tráfico total en la red (en Pbps) frente a multiplicidad espacial $(S)$ con \begin{tabular}{|c|}
\hline Spe-SCh en: (a) DT12 con TP1, (b) DT12 con TP2, (c) EON16 con TP1 \\
\hline
\end{tabular}

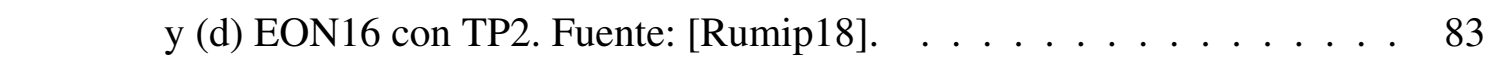

4.15. Tráfico total en la red (en Pbps) frente a multiplicidad espacial $(S)$ con \begin{tabular}{|c|}
\hline Spa-SCh en: (a) DT12 con TP1, (b) DT12 con TP2, (c) EON16 con TP1 \\
\hline
\end{tabular} y (d) EON16 con TP2. Fuente: [Rumip18]. . . . . . . . . . . . . . 84 


\section{Lista de Tablas}

1.1. Lista de objetivos para $5 \mathrm{G}(5 \mathrm{G}$ KPIs). . . . . . . . . . . . . . . 4

2.1. Lista de servicios de Internet . . . . . . . . . . . . . . . . 25

2.2. CAGR total del tráfico (y trafico cursado) para un periodo de 15 años. . . 27

3.1. Lista de VNFs en [Moren18b]. . . . . . . . . . . . . . . . . . . . 47

3.2. Lista de VNFs en $[$ Moren $18 \mathrm{~d}] . \ldots \ldots \ldots$. . . . . . . . . . . . . 53

4.1. Pérdidas introducidas por los splitters $[\mathrm{dB}]$. . . . . . . . . . . . . 69

4.2. Número de puertos de entrada por SSS . . . . . . . . . . . . . . . . . . 69

4.3. Métricas de nodo óptico para diferentes diseños SDM-ROADM . . . . . 69

4.4. Alcance óptico (en Km) y espacio espectral ocupado (en FSs) para el conjunto de transponders, según modulación, line rate y número de núcleos en la fibra. . . . . . . . . . . . . . . . . . . . . . . . . 74

4.5. Throughput total en T7S usando ILP frente al heurístico . . . . . . . . . . 78

4.6. Throughput total en I2 usando ILP frente al heurístico . . . . . . . . . . . 78

4.7. Métricas relativas a las redes de referencia . . . . . . . . . . . . . . 78

4.8. Eficiencia económica de la red para 4 topologías diferentes . . . . . . . . 79

4.9. Estimación de alcance óptico en km en fibras SDM . . . . . . . . . . . . 82

4.10. Coste normalizado de por SSS. Fuente: [Rivas17] . . . . . . . . . . . . 85

4.11. Coste de red según tamaño para distintos SDM-ROADM. . . . . . . . . . 86 



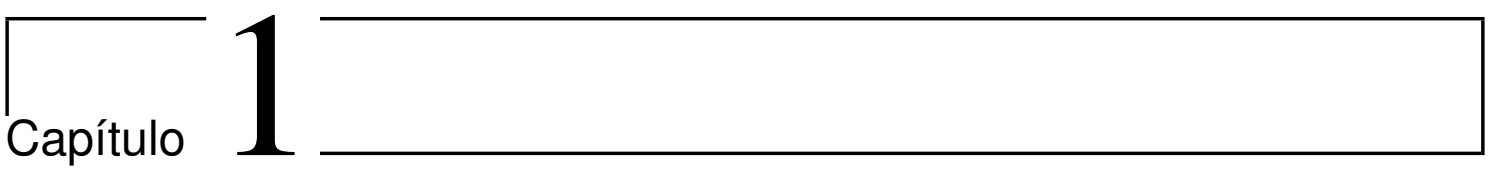

\section{Introducción}

1.1. Introducción general $\ldots \ldots \ldots \ldots \ldots \ldots$

1.2. Crecimiento del tráfico IP e impacto en las redes de transporte . . . . . . . 3

1.3. Propuestas para afrontar el Internet del futuro . . . . . . . . . . . . 4

1.4. Publicaciones derivadas de la tesis $\ldots \ldots \ldots \ldots \ldots \ldots$

1.5. Proyectos enmarcados en el desarrollo de la tesis $\ldots \ldots \ldots \ldots \ldots$

1.6. Estructura de la tesis $\ldots \ldots \ldots \ldots \ldots \ldots$

Los problemas derivados del cumplimiento de las previsiones de crecimiento exponencial del tráfico IP para los próximos años y los desafíos propuestos para la satisfacción de las propuestas del futuro paradigma $5 \mathrm{G}$ han servido como puntos de partida en la motivación de esta tesis, orientada en la búsqueda de soluciones para contrarrestar dicha problemática a la vez que se intenta satisfacer los estrictos requisitos impuestos por el futuro 5G. Esta sección introductoria trata de contextualizar la motivación que ha estimulado el desarrollo de esta tesis doctoral además de proporcionar una breve discusión sobre las posibles soluciones a evaluar en el presente trabajo. Finalmente se enuncian los proyectos y publicaciones derivados del marco de la tesis.

\subsection{Introducción general}

Las redes de telecomunicaciones han evolucionado a tal escala que la sociedad y la economía se han vuelto totalmente dependientes de su existencia. El gran conjunto de redes globales que conforma Internet ha creado un caldo de cultivo idóneo para establecer comunicación a distintos niveles entre puntos muy remotos del globo terrestre aportando experiencias casi instantáneas. Este hecho, añadido a la gran proliferación de servicios basados en la nube, ofrece a usuarios de todo el mundo experimentar un conjunto de servicios como nunca antes se había visto. Como ejemplo, un usuario en Australia puede visualizar contenido audiovisual original creado por otro usuario desde Suecia en ultra alta definición sólo unos pocos segundos después de su creación o incluso visualizar retransmisiones deportivas en Estados Unidos en riguroso directo con un retardo de unos 
pocos segundos, sin necesidad de utilizar satélites geoestacionarios, gracias a la tecnología derivada de la fibra óptica. Por otra parte, la caída temporal de las grandes redes de comunicaciones puede conllevar a un colapso social y económico de cifras alarmantes.

Lejos de estacionarse, las redes de telecomunicaciones siguen evolucionando con la promesa de aportar nuevas experiencias a los usuarios y mejorar el estado del bienestar. La $5^{\text {a }}$ generación de redes móviles, llamada $5 \mathrm{G}$, promete establecer comunicaciones con latencias en canal radio menores a $1 \mathrm{~ms}$, hecho especialmente orientado a amplias zonas urbanas [5Gkpi]. La emergencia de este próximo paradigma en Internet, establecerá nuevos horizontes a los servicios actuales y abriendo la puerta a nuevas aplicaciones. La satisfacción de los requerimientos prometidos para 5G habilitará un nuevo contexto llamado, por la Unión Internacional de Telecomunicaciones (ITU por sus siglas en inglés), como Internet Táctil, definido por una combinación de muy baja latencia, seguridad y confianza en la utilización de aplicaciones en campos como industria automovilística, sistemas de transporte orientados a la ayuda sanitaria, educación o videojuegos [ItuT14]. Las previsiones derivadas del contexto del Internet Táctil marcan gran impacto a nivel social y económico introduciendo numerosas oportunidades para tecnologías emergentes y prestación de servicios esenciales a la sociedad, acercando notablemente el contenido a los usuarios.

Sin embargo, este gran aporte de beneficios proporcionado por el actual o futuro paradigma de Internet tiene implicaciones que pueden ser dramáticas. El aumento en la calidad y cantidad de servicios basados en la nube aportan un gran volumen de tráfico añadido que puede llegar a desbordar la capacidad actual que tienen las redes de comunicaciones para servir dicho tráfico. Sin ir mas lejos, según un informe de octubre de 2018 proporcionado por Sandvine, una única aplicación como Netflix, que sirve contenido de vídeo en ultra alta definición, acapara casi una quinta parte de todo el tráfico que se mueve por redes estadounidenses [Sandv18]. Por otro lado, las previsiones aportadas por el informe Cisco VNI de 2017 apuntan a un posible crecimiento anual en el tráfico del $27 \%$ [Cisco17]. Este contexto conjunto, de proliferación de servicios basados en la nube, especialmente vídeo bajo demanda, además de las previsiones de crecimiento de tráfico asociado a dichos servicios crean una imagen de gran incertidumbre para el estado actual de las redes ópticas de telecomunicaciones.

Los principales objetivos de esta tesis doctoral son investigar, en primera instancia, la validez de las elevadas predicciones de crecimiento tráfico teniendo en cuenta la naturaleza de las actuales aplicaciones y la tendencia de acercar el contenido a los usuarios, según las redes de acceso $5 \mathrm{G}$ o en las redes de distribución de contenido, así como su impacto en las grandes redes ópticas. En segundo lugar, la tesis estudia soluciones que aborden la anterior problemática de crecimiento de tráfico desde tres grandes perspectivas: a) optimización en el diseño de las redes de reparto de contenido, b) una mejor gestión, flexibilidad y programabilidad característica de las redes controladas por software (SDN, Software-Defined Networking) y la virtualización en las funciones de la red (NFV, Network Function Virtualization) y finalmente, c) cómo el aumento de nuevos canales espaciales en las redes de fibra óptica dado por la tecnología SDM (Space Division Multiplexing) puede expandir la capacidad de las redes de transporte. 


\subsection{Crecimiento del tráfico IP e impacto en las redes de transporte}

Para contextualizar el impacto de Internet alcanzado en la sociedad a principios del siglo XXI, el informe "Cisco Visual Networking Index: Forecast and Methodology, 20162021" proporciona un conjunto de conclusiones derivadas al tráfico de Internet, algunas de las mas relevantes se exponen a continuación: a) mayor volumen, se espera que para 2021 el tráfico total de Internet alcance $\operatorname{los} 3.3 \mathrm{ZB}\left(3.3 \cdot 10^{21} \mathrm{~B}\right)$, mientras que para $2016 \mathrm{el}$ tráfico total era de $1.2 \mathrm{ZB}, \mathrm{b}$ ) rápido crecimiento, el tráfico IP (Internet Protocol) se prevé que crezca a un ritmo anual del $27 \%$, c) perfiles de tráfico mas agresivos, la hora central de tráfico crece a mayor ritmo que el tráfico promedio y d) mayor movilidad, se espera que en 2021 el volumen de tráfico inalámbrico derivado del uso de aplicaciones móviles o similar superará al tráfico fijo, un $63 \%$ del total [Cisco17].

Por otra parte, el concepto de nube es totalmente determinante para entender el actual paradigma en las redes de comunicaciones. Se entiende como la nube al concepto abstracto que integra a un conjunto global de servidores donde las aplicaciones alojan su contenido para poder ofrecer a los usuarios los servicios asociados a dichas aplicaciones [Micros18]. Tales servidores están diseñados para el almacenamiento y administración de datos, de tal manera que se puedan ejecutar las aplicaciones de forma remota sin la necesidad de tener un gran volumen permanente de datos por parte del usuario. Algunas compañías ofrecen parte de su infraestructura como servicio (Infrastructure as a Service, IaaS), algunos ejemplos relevantes de dichas infraestructura son la red Azure de Microsoft, Amazon Web Services, Akamai, Limelight o Level3. Este entorno amigable para el desarrollo de las aplicaciones ha derivado en una inmensa cantidad de tráfico proveniente del intercambio de contenido en tales aplicaciones. Según el informe "The Global Internet Phenomena Report 2018” de Sandvine, el $58 \%$ del total de tráfico de Internet deriva de un solo tipo de servicio, vídeo bajo demanda, donde la aplicación Netflix se encarga del $15 \%$ del volumen mundial de tráfico de bajada, mientras que el tráfico de subida está mayoritariamente representado por las aplicaciones P2P (Peer-to-peer), con BitTorrent como su mayor representante y $22 \%$ el tráfico total de subida [Sandv18]. Las aplicaciones basadas en redes sociales, como Instagram, Facebook o Snapchat, también son un fenómeno digno de mención que genera un gran intercambio continuo de tráfico donde los usuarios descargan y suben contenido constantemente. Instagram copa el $42 \%$ del tráfico total asociado a redes sociales.

Es muy probable que el contexto global de Internet cambie con la imposición de la llamada $5^{\mathrm{a}}$ Generación de redes móviles, 5G. Según el consorcio que conforma 5G PPP (5G Public-Private-Partnership), "5G es un ecosistema de extremo a extremo que permite una sociedad totalmente móvil y conectada. Facilita el acceso de contenido hacia usuarios y propietarios, a través de casos de uso existentes y emergentes, entregados a una experiencia consistente y habilitada por modelos de negocio sostenibles" [5Gkpi]. Los estrictos objetivos que pretende conseguir en contexto 5G (5G KPIs, Key Performance Indicators), bajo recomendaciones de la ITU, se exponen en la siguiente Tabla 1.1 . 
Tabla 1.1: Lista de objetivos para 5G (5G KPIs).

(URLLC, Ultra Reliable Low Latency Communications, eMBB, enhanced Mobile BroadBand, mMTC, massive Machine Type Communications). Fuente: [5Gkpi].

\begin{tabular}{|l|l|}
\hline \multicolumn{1}{|c|}{ Tipo de Requerimiento } & \multicolumn{1}{c|}{ Objetivo (KPI) } \\
\hline Tasa de datos máxima & $\begin{array}{l}\text { Bajada: } 20 \mathrm{Gbps}, \\
\text { Subida: } 10 \mathrm{Gbps}\end{array}$ \\
\hline Eficiencia espectral máxima & $\begin{array}{l}\text { Bajada: } 30 \mathrm{~b} / \mathrm{s} / \mathrm{Hz}, \\
\text { Subida: } 15 \mathrm{~b} / \mathrm{s} / \mathrm{Hz}\end{array}$ \\
\hline Tasa de datos en el usuario & $\begin{array}{l}\text { Bajada: } 100 \mathrm{Mbps}, \\
\text { Subida: } 50 \mathrm{Mbps}\end{array}$ \\
\hline & $\begin{array}{l}\text { Interior: B:0.3/S:0.21, } \\
\text { Urbano: B:0.225/S:0.15, } \\
\text { Rural: B: } 0.12 / \mathrm{S}: 0.045 \\
\text { (en b/s/Hz/TRxP) }\end{array}$ \\
\hline centil 5 & \\
\hline Eficiencia espectral promedio & $\begin{array}{l}\text { Interior: B: } 9 / \mathrm{S}: 6.75 \\
\text { Urbano: B: } 7.8 / \mathrm{S}: 5.4 \\
\text { Rural: B: } 3.3 / \mathrm{S}: 1.6 . \\
\text { (en b/s/Hz/TRxP) }\end{array}$ \\
\hline Latencia plano usuario & $\begin{array}{l}4 \mathrm{~ms} \text { en } \mathrm{eMBB} \\
1 \mathrm{~ms} \text { para URLLC }\end{array}$ \\
\hline Latencia plano control & $20 \mathrm{~ms}(\mathrm{o} 10 \mathrm{ms)}$ \\
\hline Densidad de conexión & $10^{6}$ disp/km ${ }^{2}$ (mMTC) \\
\hline
\end{tabular}

La conjunción del estado actual del paradigma de Internet, con las previsiones de crecimiento de tráfico IP y los objetivos a cumplir para las redes 5G, habilitan un panorama que puede ser complejo para las redes de comunicaciones creando una perspectiva nunca vista hasta la fecha. La evolución futura de las redes de telecomunicaciones reside, en esencia, en la capacidad de adaptación que se tenga al cumplimiento de las anteriores predicciones. Para anticipar a tal contexto, es necesario abordar posibles soluciones para solventar total o parcialmente los problemas relativos a la aparición del futuro entorno. Las tecnologías que se han analizado en la presente tesis doctoral como posibles soluciones que afronten los desafíos futuros se exponen en la siguiente sección.

\subsection{Propuestas para afrontar el Internet del futuro}

El paradigma actual del tráfico IP, basado en gran medida en aplicaciones en la nube y sumado a las altas expectativas puestas en el futuro contexto $5 \mathrm{G}$, motivan las previsiones de tráfico con un considerable crecimiento. Este panorama es preocupante ya que puede congestionar la infraestructura global de redes de comunicaciones [Napol15][Jia17]. Para intentar seguir el paso al crecimiento del tráfico, numerosos esfuerzos en el mundo académico han analizado posibles soluciones para afrontar tales desafíos. En la presente tesis doctoral se investigan tres grandes enfoques: a) planificación de redes de distribución de contenido (CDN), b) centralización y optimización de recursos de red y virtualizados mediante software y c) la expansión de la capacidad de las redes según las tecnologías 
flex-grid y SDM.

\subsubsection{Descongestión de redes troncales, planificación de CDNs}

El tráfico IP actual está compuesto en una gran mayoría de servicios basados en la nube ofrecidos por un enorme número de aplicaciones, siendo dicha cantidad cada vez mas alta. Normalmente, las aplicaciones basadas en la nube ofrecen sus servicios a través de un conjunto de redes llamadas redes de distribución de contenido o CDNs (Content Delivery Networks). Un CDN se entiende como un conjunto de granjas de datos, o datacenters (DCs), interconectados por enlaces ópticos en propiedad o alquilados a compañías que ofrecen infraestructura óptica para ser utilizada. Muchas de la principales compañías que monopolizan el tráfico a nivel de aplicación tienen en propiedad o en desarrollo sus propios CDNs. Algunos casos muy representativos son Google [Googl], Amazon [Aws], Microsoft [Micros18].

Crear nuevos datacenters, expandiendo el tamaño de los CDNs, puede tener implicaciones directas en la congestión de las grandes redes ópticas (core/backbone). La hipótesis de que la proliferación de CDNs puede aliviar la carga en las redes ópticas de transporte reside en el hecho de que al acercar el contenido a los usuarios el camino óptico desde el origen del contenido hasta su destino se puede reducir notablemente e incluso desparecer en el caso de que el CDN pueda servir su tráfico sin atravesar las redes ópticas core/backbone. Por otro lado, un mayor reparto de contenido implica mayor tráfico de sincronización entre las réplicas de dicho contenido, pudiendo aportar mayor carga a la red core. Cómo se reparta el contenido dentro del CDN, así como su tamaño, pueden ser determinantes en una correcta planificación de red. En esta tesis se intentará arrojar luz sobre la incertidumbre asociada a las posibles implicaciones de una correcta planificación del CDN en la congestión del core así como la validación del cumplimiento hipotético de las predicciones de crecimiento de tráfico.

\subsubsection{Optimización de recursos de red e IT, SDN-NFV}

El amplio conjunto de servicios basados en la nube ha dado lugar a un nuevo contexto de planificación y gestión de redes de telecomunicaciones donde los recursos de red y los recursos computacionales, CPU (Central Processing Unit), RAM (Random Access Memory) y HDD (Hard Drive Device), coexisten conjuntamente para poder ofrecer los servicios de las aplicaciones. El entendimiento de este contexto es básico para el futuro cumplimiento de los objetivos $5 \mathrm{G}$ y se presenta como una evolución orgánica de las técnicas tradicionales de gestión y control. Como solución a tales desafíos, la gestión y configuración de la red a través de software (SDN) y la virtualización de las funciones de red (NFV) emergen como dos tecnologías muy prometedoras que pueden aportar la centralización, flexibilidad y programabilidad que se requieren en los 5G KPIs.

A su vez, el uso de herramientas en código abierto que permitan la colaboración abierta de desarrolladores en el marco SDN/NFV puede favorecer de manera notable la investigación y el desarrollo cooperativo a nivel global acelerando la llegada de los futuros beneficios de las tecnologías SDN y NFV. 
Una parte muy importante de la presente tesis se centra en analizar la bondad de las tecnologías SDN/NFV en la gestión y control de redes con vistas a la optimización en dicho entorno, siempre bajo el uso de herramientas open source.

\subsubsection{Expansión de redes ópticas, flex-grid y SDM}

El crecimiento de tráfico de Internet previsto para los próximos años puede sobrepasar la capacidad actual de las infraestructuras ópticas. Por está razón, entre otros motivos, la eficiencia y expansión de las redes troncales parecen ser necesarias para afrontar tales desafíos.

Las redes elásticas flex-grid aportan mejor utilización del espacio espectral, que unido a la mayor capacidad espacial proporcionada por la tecnología SDM, puede aumentar no solo la capacidad de la red, sino además una gestión mas eficiente de los recursos espectrales y espaciales. La creación de súper canales, tanto espaciales como espectrales en el contexto flex-grid/SDM, habilita la transmisión de datos a un nivel sin precedentes. Esta tesis doctoral estudia dicho contexto como posible opción para soportar las futuras implicaciones en los enlaces de las redes core o backbone derivadas del cumplimiento de las predicciones.

Este trabajo está conformado, en parte, por estudios, discusión, propuestas y resultados contextualizados en redes elásticas con soporte de fibras SDM/flex-grid.

\subsection{Publicaciones derivadas de la tesis}

Esta sección resume la contribución total de la tesis desde la perspectiva de publicaciones en revistas especializadas o en trabajos presentados en congresos de difusión internacional. Tal contribución se puede diferenciar en tres grandes bloques temáticos: a) estado y evolución de las redes core, b) gestión y optimización de redes basadas en SDN/NFV y finalmente, c) expansión de capacidad de redes ópticas elásticas según SDM y flex-grid.

En primer lugar, el estudio de la problemática a nivel de congestión de las redes troncales y su relación con la evolución de las redes de distribución de contenido (CDNs) ha sido estudiada principalmente en dos trabajos. El primero de ellos es un artículo presentado en el congreso internacional OCF17 (Los Ángeles, EEUU) en el que se investiga cómo la creación de nuevos centros de datos en redes tipo CDN puede parcialmente aliviar la congestión en las grandes redes ópticas [Pavon17]. Por otro lado, el artículo en revisión en la revista Computer Networks hereda el trabajo anterior para realizar un estudio mucho mas profundo sobre la interdependencia del estado de las redes core con el tráfico generado en los servicios basados en la nube [Moren18a]. Los resultados han mostrado tres grandes conclusiones, (i) acercar el contenido a los usuarios puede parcialmente reducir la carga en el core mientras crece considerablemente el coste para los propietarios de los CDNs, (ii) un reparto eficiente del contenido en el CDN puede descongestionar de forma significativa el impacto en las grandes redes ópticas y por otro lado, (iii) la naturaleza de los futuros servicios se presenta como determinante en la evolución de las redes de transporte. 
Segundo, el control de redes mediante software y la virtualización de redes han sido los protagonistas en el segundo gran bloque de la tesis. Se ha presentado en el congreso OFC18 (San Diego, EEUU) una demostración donde se muestra una optimización de cadenas de servicio utilizando herramientas open source en un contexto NFV [Moren18b]. Una extensión de tal trabajo, se presentó en la demo zone del congreso ECOC18 (Roma, Italia), donde se tiene en cuenta también el plano de datos gracias a la integración de un controlador SDN [Moren18d] con el fin de minimizar la latencia total. De nuevo, en el congreso OFC18 se presentó un tutorial invitado en el que el candidato ha participado como coautor. Dicho trabajo trata sobre el papel de la optimización en redes basadas en SDN/NFV [Pavon18], que además sirvió como piedra angular para la expansión publicada en la revista Journal on Lightwave Technology [Garri18a].

El último bloque temático aborda la ampliación de la capacidad en redes ópticas desde la perspectiva de la tecnología SDM, donde gracias a una nueva dimensión de multiplexación en la fibra óptica proporcionado por la tecnología SDM se consigue un aumento de la capacidad de la red y una mejor eficiencia espectral proporcionado por el paradigma flex-grid. Las publicaciones presentadas giran en torno a una propuesta principal, la continuidad del canal espacial en redes SDM. Esta idea se evaluó inicialmente en un artículo presentado en el congreso ONDM17, donde además se presentan las ventajas de uso de un ROADM basado en esta idea [Rumip17a]. Dicho trabajo se expande sustancialmente en el análisis off-line en un contexto MCF (Multi Core Fiber) publicado en la revista Journal of Optical Communications and Networking [Moren17]. Por otra parte, la idea de continuidad es puesta a prueba satisfactoriamente en un marco dinámico en el artículo publicado en la revista Computer Communications [Rumip18]. Finalmente, [Moren18c] resume los logros del proyecto Elastic Networks, donde se incluyen los obtenidos en el campo SDM/flex-grid anteriormente mencionados.

\subsection{Proyectos enmarcados en el desarrollo de la tesis}

El desarrollo de la presente tesis ha sido posible gracias a la financiación y el marco de diversos proyectos nacionales e internacionales. Esta sección trata de enunciar los proyectos en los que el candidato ha formado parte activa, así como una descripción del contexto, objetivos principales y contribución académica del desarrollo de la tesis en cada proyecto.

\subsubsection{Proyecto ONOFRE}

Los pronósticos de crecimiento exponencial del tráfico en las redes de telecomunicaciones, debido en gran parte a la creciente popularidad de los servicios o computación basados en la nube, redes sociales o juegos en línea, amenazan la eficiencia económica de las grandes redes de comunicación a nivel global. Las predicciones de tráfico además anuncian un aumento en la movilidad y diversidad en la naturaleza del tráfico aportando un nuevo grado de complejidad a la gestión tradicional de las redes. Tal marco está orientando en la dirección del desarrollo de las tecnologías ópticas en las redes de acceso y la adopción de las redes IP sobre capa óptica. 
Bajo tal contexto, el proyecto Optical Networks Convergence in The Future Internet, ONOFRE, [Onofr], se ha centrado en un diseño convergente de redes de acceso o metropolitanas que enmarca la investigación con el objetivo de gestionar de manera eficiente la gran variabilidad del tráfico en múltiples capas. La gestión eficiente del plano de control a través de software es un tema capital para este proyecto.

El proyecto Optical Networks Convergence in The Future Internet, ONOFRE, es un proyecto nacional (referencia TEC2014-53071-C3-1-P) perteneciente al Programa Estatal de Fomento de la Investigación Científica y Técnica de Excelencia, Convocatoria de ayudas a Proyectos de I+D Excelencia Subprograma de Generación de Conocimiento 2014 cuya responsabilidad recae en la Secretaría de Estado de Investigación Desarrollo e Innovación dependiente del Ministerio de Economía y Competitividad del Gobierno de España. El periodo de actividad de este proyecto ha sido de tres años, entre 2015 y 2018.

El proyecto ONOFRE ha sido coordinado por el grupo GIRTEL de la Universidad Politécnica de Cartagena (UPCT) y conformado además por el grupo de comunicaciones ópticas de la Univesidad de Valladolid (UVa) y la Fundación i2CAT. El proyecto ONOFRE ha sido vital en la financiación del trabajo contenido en la presente tesis proporcionando el contexto necesario para la ayuda FPI (Formación de Personal Investigador), la cual ha sido beneficiario el candidato de la presente tesis. El resultado de este proyecto ha sido tal que ha propiciado la creación de un nuevo contexto de financiación a la investigación, ONOFRE-2.

Las contribuciones académicas de la tesis al proyecto ONOFRE se exponen en las siguientes publicaciones: [Rumip17a], [Moren17], [Pavon17], [Rumip18].

\subsubsection{Proyecto ONOFRE-2}

El proyecto Optical Networks Convergence in The Future Internet-2, ONOFRE-2 [Onof2], amplía los objetivos propios de la propuesta de red acceso/metro dados por su precursor, el proyecto ONOFRE, orientados a fomentar el uso de virtualización en las redes (NFV), centralizar el plano de control de red (SDN) prometiendo una reducción tanto en capital (equipamiento) como en coste operacional. Este nuevo contexto abre la puerta al uso de tecnologías emergentes como Internet de las cosas (Internet of Things, IoT), Industria 4.0 o Internet Táctil. La virtualización, unida al uso del paradigma Multi Edge Computing (MEC), permitirá reducciones en el consumo de energía y en costes CAPEX y OPEX, además de habilitar nuevos servicios de Internet de las Cosas o Internet Táctil. Las principales novedades de esta extensión del proyecto ONOFRE son: a) una propuesta de la arquitectura acceso/metro pueda satisfacer los requerimientos de los servicios 5G, IoT o Internet táctil, b) la integración de computación distribuida (MEC) para poder lograr los objetivos anteriores y c) el uso de técnicas basadas en NFV y SDN para aportar mayor nivel de control, flexibilidad y programabilidad a la red.

ONOFRE-2 está liderado por la el grupo de comunicaciones ópticas (GCO) de la UVa, proyecto en el que el grupo GIRTEL de la UPCT es miembro además de la Fundación i2CAT. Referencia: TEC2017-84423-C3-1-P (Programa Estatal de Fomento de la Investigación Científica y Técnica de Excelencia, Subprograma Estatal de Generación de Conocimiento). El proyecto empezó el 1 de enero de 2018 y tiene una duración de tres años. Las publicaciones mas relevantes que se han desarrollado como parte de la te- 
sis dentro del marco del proyecto ONOFRE-2 son: [Moren18a], [Garri18a], [Moren18b] [Moren18d], [Pavon18].

\subsubsection{Red de excelencia Elastic Networks}

De nuevo, la preocupación por las implicaciones derivadas al crecimiento del tráfico en las redes de telecomunicaciones sirve de lanzadera para contextualizar el objetivo de la red de excelencia Elastic Networks [Elast]. Este marco de colaboración investigadora ha contribuido a abordar los desafíos anteriores focalizando los esfuerzos en hacer redes más flexibles, ágiles y programables, siempre con la vista puesta en aumentar el rendimiento mientras se reduce el coste de operación, despliegue y mantenimiento de la red.

Elastic Networks es un proyecto nacional (TEC2015-71932-REDT) perteneciente al programa "Redes de excelencia" enmarcado en el Programa Estatal de investigación Científica y Técnica de Excelencia, Subprograma Estatal de Generación de Conocimiento. La red de excelencia Elastic Networks ha sido coordinada la Universidad Carlos III de Madrid (UC3M), y conformado como miembros por la UPCT, la Universidad Politécnica de Cataluña (UPC), la Universidad Politécnica de Madrid (UPM), Universidad de Girona, la Universidad Antonio de Lebrija (UAN), Universidad Politécnica de Valencia (UPV) y el centro tecnológico de telecomunicaciones de Cataluña (CTTC). El proyecto ha proporcionado un espacio de colaboración común para afrontar los desafíos propios del aumento del tráfico IP desde la perspectiva tecnológica de las redes ópticas elásticas. La duración del proyecto ha sido de tres años, desde 2015 hasta 2018.

El marco de la red de excelencia ha contribuido en algoritmos multicapa con tres modos de recuperación, algoritmos de planificación de redes metropolitanas con tecnología filterless y tráfico multi horario. También se han realizado estudios sobre simulaciones en escenarios dinámicos 5G y aprovisionamiento de NFV utilizando infraestructuras de redes elásticas. Se han propuesto y evaluado diseños de ROADM para un escenario de redes elásticas con fibras $\mathrm{MCF}$, donde se ha apreciado un gran beneficio en el balance rendimiento-coste respecto de otros diseños de referencia. Los resultados de esta colaboración han abierto la puerta a una posible continuación de la propia red temática de excelencia. Las contribuciones académicas en las que ha participado el doctorando dentro del contexto de tesis han sido: [Rumip17a], [Moren17], [Moren18c] y [Rumip18].

\subsubsection{Metro-Haul H2020 Project}

El principal objetivo del proyecto Metro-Haul [Metro] es diseñar y construir una infraestructura óptica para redes metropolitanas que sea capaz de soportar el tráfico creado en las redes de acceso 5G, cumpliendo los objetivos propios del paradigma 5G (muy baja latencia, movilidad, variabilidad de servicios, baja fluctuación, etc.). Tales objetivos se pretenden alcanzar a través de una nueva propuesta de arquitectura de red basada en nuevos tipos de nodo, de acceso (AMEN) y metro (MCEN), con capacidad de almacenamiento y cómputo, que integren los conceptos de desagregación de hardware, virtualización (NFV), automatización y programabilidad (control SDN).

Metro-Haul es un proyecto del programa H2020 (H2020-ICT-2016-2 G.A. 761727) de la Unión Europea. El proyecto está conformado por un total de 21 entidades públicas y 
privadas, donde se pueden encontrar como miembros compañías de la índole de Telefónica, ADVA, Frahunhofer HHI, Nokia o Telecom Italia, además de entidades investigadoras de la talla de CNIT, CTTC o University of Bristol. El grupo GIRTEL de la UPCT está presente en el proyecto Metro-Haul como third party de la UPC. El proyecto se inició en 2017 y tiene una duración de tres años. Las contribuciones académicas en las que ha participado el candidato dentro del contexto de tesis han sido [Moren17], [Moren18a], [Moren18b],[Moren18d], [Garri18a].

\subsection{Estructura de la tesis}

En esta sección se resume el contenido del resto de este documento:

- El capítulo dos se contextualiza en el marco de un estudio para mostrar el impacto de las redes troncales cuando se asume el crecimiento y expansión de las redes de distribución de contenido (CDNs), teniendo en cuenta las predicciones de tráfico de [Cisco17] y [Sandv16] en dicha evolución y un modelo de tráfico que asume la naturaleza particular de diversos servicios basados en la nube.

- El capítulo tres aborda una posible solución para minimizar el impacto del crecimiento de tráfico desde la perspectiva de una gestión óptima y centralizada de redes basadas en SDN y NFV. En dicho capítulo, se muestra el papel de las herramientas de código abierto en el proceso de gestión y optimización de redes SDN-NFV. Finalmente, se exponen las descripciones y conclusiones derivadas de dos pruebas de concepto en un entorno SDN-NFV utilizando sólo herramientas open source.

- El capítulo cuatro estudia el uso de la tecnología SDM como opción para aumentar la capacidad de los enlaces que componen las redes de comunicaciones ópticas y de esta forma afrontar el problema de crecimiento previsto de tráfico. Se ha propuesto la idea de continuidad en el canal de multiplexación como posible opción en el desarrollo de prototipos de nodos ópticos. Su rendimiento se ha puesto bajo prueba en entornos flex-grid de tráfico estático y dinámico.

- El capítulo cinco presenta las principales conclusiones obtenidas en la realización de la presente tesis doctoral así como una discusión de las mismas orientada a trabajos futuros. 


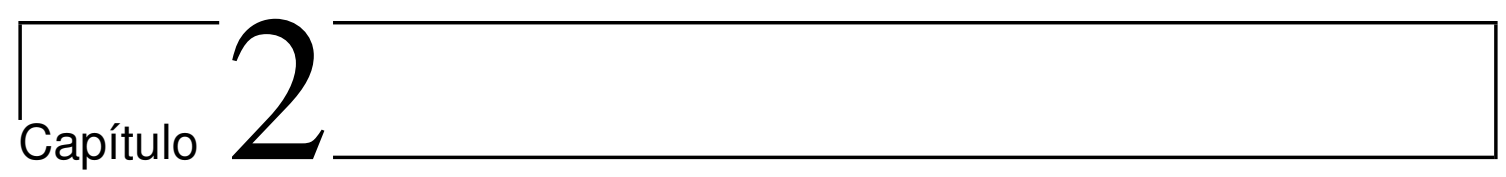

\section{Estado y Evolución de Redes Troncales}

2.1. Introducción y estado del arte $\ldots \ldots \ldots \ldots \ldots$

2.2. Problemática e hipótesis inicial $\ldots \ldots \ldots \ldots \ldots \ldots \ldots$

2.3. Modelo de evolución temporal de red $\ldots \ldots \ldots \ldots$

2.4. Resultados numéricos $\ldots \ldots \ldots \ldots \ldots \ldots \ldots$

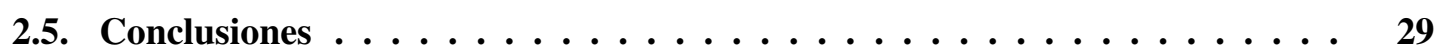

En este capítulo se presenta un análisis y discusión del estado actual y la evolución de las redes troncales de transporte en telecomunicaciones, conocidas como redes core o backbone, desde la perspectiva de las previsiones de tráfico IP, modelado como servicios asociados a aplicaciones utilizadas por usuarios remotos. Con el fin de poner en contexto la problemática actual de crecimiento del tráfico IP sobre el impacto en las redes de transporte, se ha realizado un estudio sobre la posible evolución de los diferentes tipos de tráfico IP, junto con sus correspondientes tipos de encaminamiento para la distribución de tráfico y para varias políticas de crecimiento en redes de distribución de contenido (Content Delivery Networks, CDNs). El algoritmo propuesto para llevar a cabo las simulaciones forma parte del cuerpo del presente trabajo.

El trabajo incluye un estudio paramétrico poniendo el foco en la naturaleza de los servicios desde el punto de vista del tráfico servido al usuario respecto del coste en sincronización interna del contenido a lo largo del CDN. Sumando las contribuciones de ambas partes se concluye que el futuro de las redes troncales tendrá una dependencia inequívoca de la naturaleza de los servicios.

Este capítulo engloba las contribuciones presentadas en los siguientes trabajos: [Pavon17], presentado en el congreso internacional OFC18, Los Ángeles (EEUU) y [Moren18a], una evolución del anterior trabajo en proceso de revisión en la revista Elservier Computer Networks. 


\subsection{Introducción y estado del arte}

Las predicciones en los últimos años anuncian un crecimiento sostenido del tráfico core, principalmente debido a la expansión de los servicios en la nube y a la gran cantidad de datos soportados por las redes de distribución de contenido [Cisco16a] [Cisco17][Sandv16] [Napol15] que puede traducirse en un aumento de la congestión en los enlaces de las redes core y backbone [Jia17]. En la Figura 2.1] se aprecia la tendencia exponencial derivada (con crecimiento anual acumulado del $27 \%$ ).

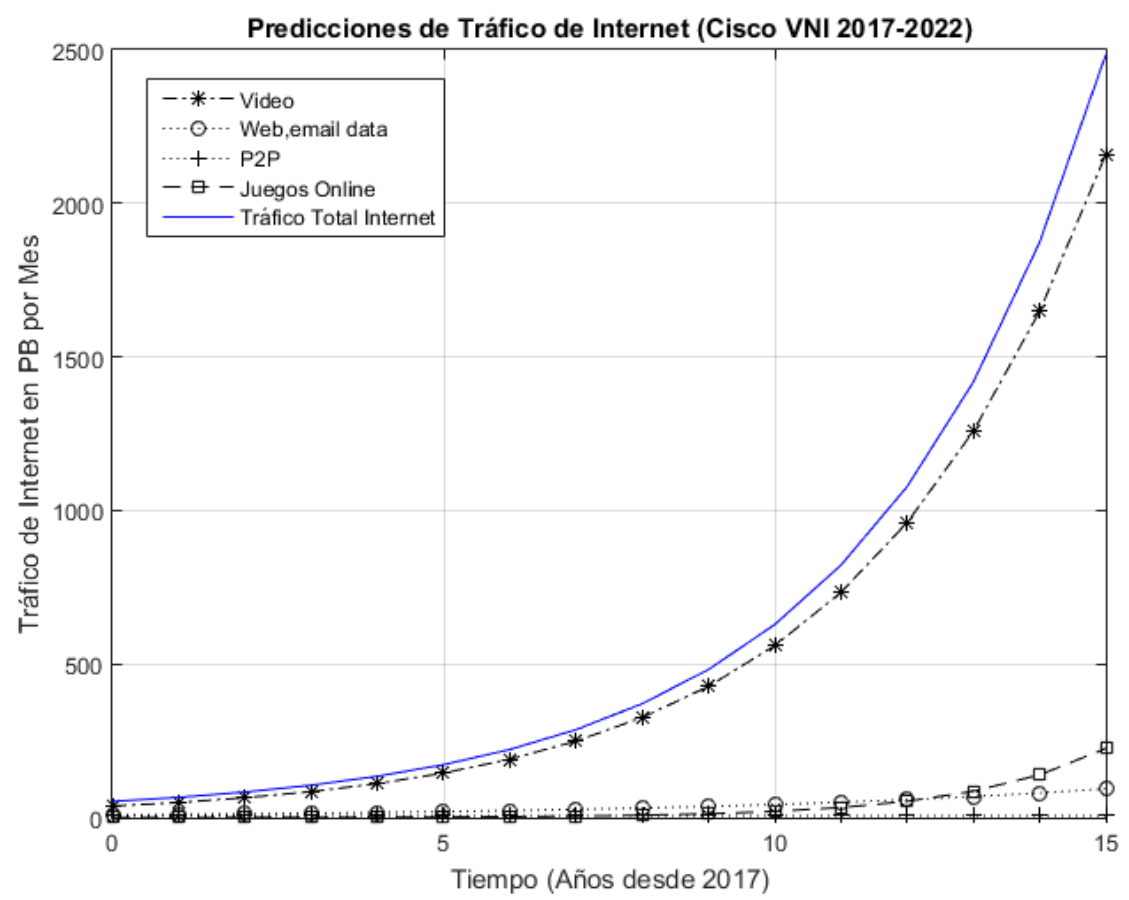

Figura 2.1: Predicciones de tráfico IP según [Cisco17] para distintos servicios y el total de tráfico IP.

Para hacer frente a tales desafíos, la comunidad científica investiga en diversos campos la forma de aliviar y optimizar la carga en los enlaces de los principales CDNs. Una de estas emergentes tecnologías es SDM (Space Division Mutliplexing), donde, gracias a un mayor grado de multiplexación por cada fibra, es posible aumentar la capacidad de los enlaces sin crear nuevas infraestructuras [Moren17][Rumip17a] [Rumip18]. SDN y NFV, son dos de las tecnologías mas prometedoras de la actualidad, no solo para abordar a la problemática de crecimiento de tráfico, sino también para acercarse a las futuras expectativas de rendimiento en 5G [Garri18a]. SDN separa el plano de datos respecto del plano de control para aportar flexibilidad controlada mediante software, mientras que NFV se centra en la implementación de funciones de red tradicionales en máquinas virtuales alojadas en microdatacenters dispersos en la red [Pavon18]. Las redes elásticas proporcionan flexibilidad y una mejor gestión de los recursos espectrales en las redes ópticas [Lopez16]. Los anteriores campos de estudio vienen impulsados por la necesidad de afrontar las predicciones en el crecimiento del tráfico. Bajo tal entorno, este capítulo investiga la posible 
evolución las redes core/backbone con presencia de redes de distribución de contenido, así como su impacto en la posible evolución del tráfico.

Normalmente, el tráfico de las aplicaciones que ofrecen servicios basados en la nube es gestionado y servido por redes especialmente diseñadas para la distribución de contenido, los CDNs. Un CDN es un conjunto de grandes centros de datos o datacenters (DCs) interconectados a través de enlaces. El contenido de las aplicaciones asociadas a servicios cloud es almacenado en los DCs, normalmente localizados en la cercanía de nodos troncales para dar acceso al resto de la red core o backbone. En ocasiones, la misma empresa que ofrece el contenido de las aplicaciones, llamada Content Provider (CP), es propietaria del CDN, como pueden ser Facebook o Google. En otros casos, los CPs alquilan parte de la infraestructura a los propietarios de los CDNs para poder realizar la distribución de contenido a los usuarios finales.

En la literatura es fácil encontrar trabajos bajo el foco de la planificación y la optimización de CDNs, así como la algoritmia de decisión de emplazamiento (datacenter) en la distribución de contenido a lo largo del propio CDN. En [Yin17] se trata ampliamente el impacto de las localizaciones de los DCs en un CDN, cuya evaluación explora el compromiso entre la reducción de latencia al acercar el tráfico al usuario mientras aumenta el tráfico como consecuencia de un mayor volumen de coste por sincronización entre los DCs. Según [Bang16], puede ser beneficioso que múltiples proveedores de contenido interconecten los CDNs beneficiándose de la colaboración entre las distintas infraestructuras. Resolver el problema de la localización de los datacenters, tratando de evitar el consumo de energía no renovable en los DCs, es el tema capital para los autores de [Wu15]. Dónde localizar los DCs y cómo repartir el contenido es la temática principal en [Ferdo15], sin embargo, en este caso, el estudio está condicionado por las localizaciones susceptibles de desastres naturales, siendo éste el factor determinante a evitar en el diseño del CDN, realizado a través de un modelo de costes basado en mercados reales. Otro ejemplo de planificación de CDNs se muestra en [Goiri11] donde, a través del análisis de costes CAPEX (CAPital EXpenditures) y OPEX (OPerational EXpenditures) en los DCs, latencia, disponibilidad de servicios y emisión de $\mathrm{CO}_{2}$, se pretende aportar optimización a la localización de los DCs.

Cómo distribuir el contenido de los servicios a lo largo del CDN es totalmente relevante para llevar un control óptimo de los recursos disponibles en la red. Distribuciones de contenido lejanas a los usuarios puede aumentar considerablemente la congestión en los enlaces. Mientras que, por otro lado, almacenar muchas réplicas del contenido, no solo aumenta el consumo de recursos en los datacenters, sino también implica un mayor volumen de tráfico de sincronización, pudiendo ser problemático según el peso y la naturaleza de los servicios ofrecidos. La comunidad investigadora tiene muy presente tal paradigma, un ejemplo de cómo abordar este asunto se puede apreciar en [Muham17], en este trabajo los autores proponen una estrategia para optimizar conjuntamente el tráfico manycast, anycast y la localización de replicas de contenido (Manycast, Anycast and Replica Placement, MARP) con el fin de encontrar soluciones óptimas y/o subóptimas para la localización de replicas a la vez que dar solución a la asignación de ruta y longitud de onda (Routing and Wavelength Assignment, RWA) para el tráfico orientado al usuario y de sincronización. La minimización del total de los recursos utilizados en redes elásticas tras solventar el RWA para tráfico anycast es la principal contribución de los autores de 
[Perel16a]. En el trabajo [Zhu16] se propone un sistema completamente distribuido para el caching y reparto de contenido en la nube con el objetivo de mejorar el rendimiento bajo el mínimo coste. Por otro lado, minimizar el ancho de banda en los enlaces y el coste del almacenamiento es la tarea principal en las técnicas propuestas para optimizar el rendimiento en los CDNs para los trabajos [Borst10] y [Manso14]. Los autores de [Sahoo17] enumeran varios algoritmos diseñados para el aprovisionamiento de contenido a lo largo de un CDN teniendo en cuenta la evaluación de algunas métricas tradicionales, como coste o calidad de servicio (Quality of Service, QoS). Para finalizar, en [Salah17], se analizan varias propuestas para tratar el aprovisionamiento de contenido para CDNs, con el foco puesto en los cambios a lo largo del tiempo en la popularidad de los propios contenidos.

Tras realizar un análisis profundo de la problemática expuesta anteriormente, se puede concluir que la mayoría de los trabajos analizados en la literatura están principalmente orientados al dimensionamiento de redes de distribución de contenido o asociados a estrategias de emplazar replicas de contenido en los distintos DCs, pero en cualquier caso no analizadas conjuntamente. En el momento del desarrollo del presente trabajo, los estudios que se presentan en esta tesis son los primeros en considerar, no solamente optimización en el dimensionado de los CDNs y el reparto de contenido, si no también el impacto de tales soluciones en la evolución del tráfico core a lo largo del tiempo así como la naturaleza de los propios servicios, los cuales están alineados con las previsiones de crecimiento del tráfico en las redes core/backbone.

\subsection{Problemática e hipótesis inicial}

En la introducción anterior se ha expuesto el problema actual existente en las redes de comunicaciones referente al aumento de tráfico y a sus posibles implicaciones. Un análisis mas extenso sobre esta problemática así como ciertas preguntas iniciales a resolver se exponen en la presente sección.

En la actualidad, en las redes de distribución de contenido (CDNs), los DCs normalmente están situados cerca de un nodo perteneciente a una red troncal que facilite el acceso a la red por parte de los usuarios, mientras que el contenido de las aplicaciones servidas es usualmente replicado en varios DCs, de tal forma que el contenido deseado se encuentre en un DC cercano al usuario final. Analizando las tendencias de tráfico en [Cisco16a] y [Cisco17], las demandas de contenido para servicios en la nube van incrementándose a lo largo del tiempo, por lo que los propietarios de los CDNs se ven forzados a expandir los datacenters existentes o crear nuevos. Desde la perspectiva de un propietario de un CDN, la apertura de un nuevo DC puede conllevar a una gran inversión de capital (CAPEX) mientras que la calidad de servicio de un gran número de usuarios puede verse favorecido, debido a la mayor proximidad al contenido, de esta manera, el número de saltos promedio para satisfacer las demandas puede reducirse y con ello, parcialmente, la congestión en los enlaces core. Sin embargo, este hecho implica mayor tráfico de sincronización entre los datacenters, aumentando los recursos consumidos en los enlaces de las redes troncales. Analizar esta compensación en detalle es esencial para el correcto proceso, no solo de expansión de CDNs, sino también para la distribución del propio contenido, es decir, cuantas replicas se crean de cada unidad de contenido y dónde se sitúan. 
Para representar el balance beneficio/coste en el reparto de contenido, se define $\beta$ como un parámetro de ajuste entre el tráfico desde el DC al usuario y el tráfico entre DCs (sincronización) para cada servicio. De tal manera que, por ejemplo, un valor de $\beta=0.1$ en un servicio implica que el total de tráfico de usuario (DC-to-user traffic) suma $10 \mathrm{Gbps}$, mientras que el tráfico asociado a tener las réplicas actualizadas y sincronizadas a lo largo del CDN (DC-to-DC traffic) es de $1 \mathrm{Gbps}$. El valor que tome el parámetro $\beta$ depende de la naturaleza de los servicios que una aplicación este sirviendo. Supongamos una aplicación que sirve un servicio de vídeo bajo demanda (Video on Demand, VoD), como puede ser Netflix, el tráfico requerido por los usuarios es considerablemente mayor que el coste en sincronización, por lo tanto, el valor de $\beta$ es bajo. Contrariamente, las aplicaciones que ofrecen servicios basados en redes sociales, como Facebook o Whatsapp, donde se crea contenido continuamente por parte de los usuarios, genera un gran volumen de tráfico de sincronización para actualizar dicho contenido, en tal caso, se asume un valor de $\beta$ alto.

Usualmente, las demandas de los usuarios son satisfechas utilizando el camino mas corto (shortest path) según el paradigma de anycast routing, donde cualquier (any) DC que tenga réplica del contenido puede satisfacer la demanda del usuario. Mientras que, por otro lado, los procesos de actualización y sincronización de contenido implican un paradigma de encaminamiento de tipo manycast routing, en este caso, se establece un árbol multicast lo mas pequeño posible que llegue a algunos (many) de los nodos disponibles en la red, aquellos que deban tener réplica. Con el fin de aclarar la problemática que se analiza en el presente capítulo, en la Figura 2.2 se muestran dos escenarios diferentes para la expansión de un CDN considerando dos aplicaciones con comportamiento opuesto.
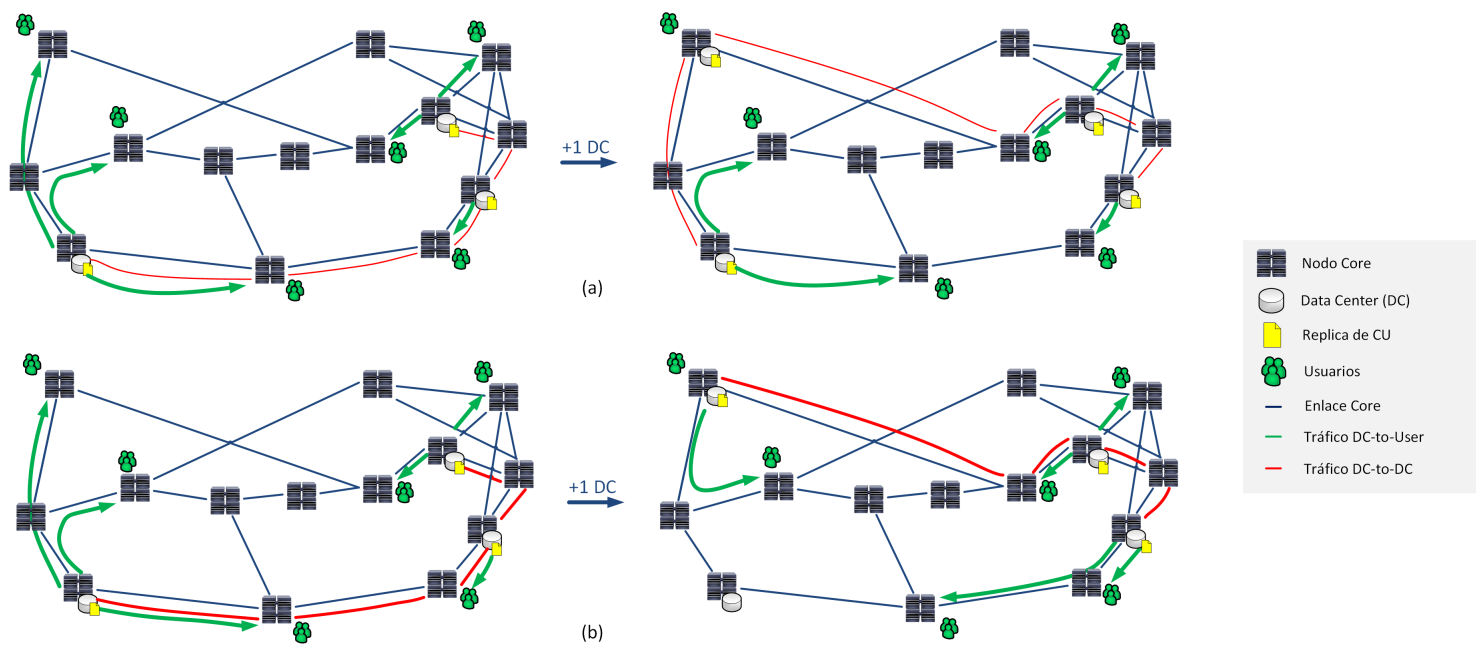

Figura 2.2: Ejemplo de expansión de $\mathrm{CDN}$ y reparto asociado de réplicas para (a) bajo $\beta$ y (b) alto $\beta$.

En la imagen 2.2(a) se asume una aplicación que sirve un servicio de bajo $\beta$, es decir, no implica un coste de sincronización alto comparado con el tráfico servido a los usuarios. Se considera que el CDN está inicialmente compuesto por tres DCs (a la izquierda) con réplica del contenido que se asume en el ejemplo, ilustrado con el icono de un documento en color amarillo. En este escenario, usuarios de seis nodos diferentes de la red demandan el contenido en cuestión, donde el coste en número total de saltos para satisfa- 
cer las demandas según anycast routing y shortest path suma 8 saltos (líneas en verde). Mientras que el coste para mantener sincronizado tal contenido en los tres DCs se realiza a través de manycast routing con un total de 5 saltos. Nótese que el grosor de las líneas del diagrama ilustra el peso relativo de cada tipo de tráfico, basado en $\beta$. Supóngase que el propietario del CDN desea crear un nuevo DC (mostrado a la derecha), por lo que una nueva réplica puede almacenarse ahí. Realizando nuevamente el encaminamiento de tráfico para satisfacer la demanda, se puede apreciar una disminución de 2 saltos en el total del tráfico relativo a los usuarios, bajo coste de incrementar en un salto el tráfico multicast como consecuencia del aumento de su respectivo árbol mínimo. Dado que para este tipo de servicio (bajo $\beta$ ) el tráfico multicast es comparativamente mucho mas bajo que el anycast, se puede afirmar que se trata de una situación ventajosa para el propietario del CDN.

Por otro lado, la Figura 2.2 (b) muestra las mismas condiciones que el ejemplo anterior pero aplicadas a un servicio con un $\beta$ alto, en este supuesto el volumen de tráfico multicast es significativo. En tal caso, el hecho de crear un nuevo DC, pero asociado a una correcta reestructuración de contenido para el mismo número total de réplicas, se consigue reducir en uno el número de saltos anycast e incluso disminuir el coste por sincronización. En este caso, el factor determinante para obtener beneficio ha sido, en mayor medida, optimizar las localizaciones de las réplicas de contenido en los DCs disponibles, hecho que se ha visto influenciado con la apertura de un nuevo DC.

Tras el análisis realizado anteriormente, se puede apreciar el impacto de la naturaleza de las aplicaciones/servicios en las redes de transporte así como la política de crecimiento del propietario de los distintos CDNs. Teniendo en mente las conclusiones anteriores, en el resto del capítulo se intentará dar respuesta a la siguiente pregunta: ¿cómo impactará la expansión de los CDNs en el crecimiento de tráfico soportado por las redes troncales?.

\subsection{Modelo de evolución temporal de red}

Con el fin de crear un marco de simulación confiable con el que se pueda ayudar a dar respuesta a la pregunta anteriormente formulada, ¿cómo impactará la expansión de los CDNs en el crecimiento de tráfico soportado por las redes core?, se ha propuesto un modelo para emular la evolución temporal de una red de transporte donde se consideran tres grandes puntos: (a) diferentes estrategias de expansión para varios CDNs que utilicen la infraestructura de la red core, (b) un modelo de tráfico IP realista que asuma las tasas de crecimiento anual (Compound Annual Growth Rate, CAGR) para distintas aplicaciones y servicios [Cisco17], y (c) un modelo que soporte el crecimiento temporal de los CDNs a la vez que optimice el aprovisionamiento del contenido de las aplicaciones servidas por tales CDNs.

En el presente trabajo, el modelo propuesto asume una red de transporte core y un conjunto de CDNs, como Amazon, Azure o Akamai. Se considera un CDN como un conjunto de DCs interconectados por enlaces de la red troncal. Aquellos CDNs que no tienen impacto en la red de transporte están fuera del estudio en el presente trabajo. Se asume un conjunto de proveedores de contenido (CPs) que aportan su tráfico en forma de aplicaciones, tipo YouTube, Instagram, Xbox Games, etc, ofreciendo un conjunto de 
servicios a lo largo de varios CDNs. Es realista asumir que un content provider utilice mas de un CDN para dar servicio a su usuarios. Por ejemplo, según el trabajo publicado en [Adhik15], Netflix utilizaba en 2015 tres CDNs distintos (Akamai, Limelight y Level3) para servir su contenido además de el CDN de Amazon para monitorización y control. El modelo propuesto también tiene en cuenta un conjunto de aplicaciones que ofrecen los tipos de servicios analizados en [Cisco17] y [Sandv16].

\subsubsection{Política de crecimiento de CDN}

El esquema de planificación multi-periodo utilizado por el modelo propuesto considera varias políticas de crecimiento para los CDNs, explicadas de la siguiente forma. En primer lugar, se asume un año cero donde los DCs de los CDNs están localizados en las proximidades de nodos core, para, de esta manera, facilitar el acceso a la red de transporte. Dado que la elección del emplazamiento de un datacenter depende de multitud de factores, como coste de la energía [Wu15], intereses político-económicos [Goiri11], resistencia a desastres naturales [Ferdo15], etc., y está fuera del contexto de este trabajo, se asignan las posiciones de los DCs de forma aleatoria. Bajo la premisa de hacer frente al crecimiento de tráfico a lo largo del tiempo, los CDNs tienen que expandirse. La política de expansión es una decisión unilateral del propietario del CDN, para modelar este comportamiento, se define un parámetro de crecimiento $G$. Supongamos un valor de $G=0$, en este caso, todo el crecimiento de tráfico es soportado los datacenters existentes, asumiendo que se refuerza la infraestructura existente, por lo que ningún nuevo DC es añadido en momento alguno. Por otro lado, para $G=1$ se propondrá abrir un nuevo DC cuando el tráfico total soportado en el CDN se doble respecto de la última apertura de un DC en ese CDN. De manera análoga, si un propietario de CDN utiliza una política de crecimiento con valor $G=2$, tenderá a crear DCs cuando el tráfico aumente un $50 \%$ respecto del último año de expansión. Es importante apuntar que si no se obtiene beneficio alguno en la apertura de un DC, se asume que el DC no ha sido creado. De esta manera también se tiene en cuenta la posibilidad de que la localización de un DC pueda no ser óptima.

Elegir la localización de un nuevo centro de datos no es una decisión trivial, sin embargo, el objetivo principal de este trabajo es investigar la expansión del CDN sobre el tráfico soportado en los enlaces de la red core. Por este motivo, se consideran dos estrategias distintas para emplazar nuevos DCs. La primera política, llamada TrOpt, trata de minimizar la carga total en los enlaces de la red core. Para tal empresa, la elección del datacenter entre los nodos candidatos será aquella que reduzca en mayor medida la suma del tráfico total anycast y multicast en los enlaces. Esta estrategia, conjuntamente con un correcto aprovisionamiento del contenido, es la opción, a priori, más idónea para aliviar el tráfico en los enlaces, sin embargo, puede ser la menos realista ya que solo considera los costes en la red de transporte. Se tiene en cuenta también una segunda estrategia para localizar los DCs, llamada Rand, donde se selecciona un nodo de forma aleatoria entre los candidatos que, para ese instante temporal, puedan reducir el tráfico total. Este último caso pretende tener en cuenta el caso donde varios factores determinan la localización de la apertura de nuevos datacenters. 


\subsubsection{Modelado de tráfico IP. Servicios y aplicaciones}

El estado actual y el futuro de las redes IP están íntimamente ligados a los servicios en la nube o cloud donde los usuarios pueden acceder a un gran conjunto de servicios a través de aplicaciones de forma remota [Cisco16a] [Sandv18]. Teniendo en cuenta el modelado de tráfico según los informes del Cisco VNI, en este trabajo se consideran tres tipos diferentes de tráfico IP para las redes core: (a) tráfico de usuario a usuario (User-touser traffic), el cual se considera independiente de los servicios cloud y es principalmente servido por las redes de los operadores, (b) el tráfico de datacenter a usuario (DC-to-user traffic), el cual tipo constituye el tráfico para servir las aplicaciones, basadas en la nube, a los usuarios que lo reclamen y por último (c) se considera un tipo de tráfico para la sincronización de réplicas y distribución del contenido entre datacenters a lo largo de los distintos CDNs (DC-to-DC traffic).

\subsubsection{Tráfico User-to-user}

El tráfico de usuario a usuario (User-to-user) se asume independiente de la existencia de CDNs o del tráfico basado en la nube. Este tipo de tráfico está modelado, para cada año de la ejecución, por una matriz cuadrada de tráfico donde el origen y destino de las demandas son previamente conocidas. Cada coordenada de la matriz indica el tráfico total que los usuarios del nodo destino reclaman al total de los usuarios del nodo origen, siendo el valor dado proporcional al producto de la población de ambos nodos. De esta manera, el flujo de tráfico entre los nodos mas poblados tendrán mayor peso que el tráfico entre los nodos con menos población. En cada iteración temporal $t$ se recalcula la matriz de tráfico en base a la tasa de crecimiento anual (CAGR) fijo para este tipo de tráfico concreto [Cisco17] según la siguiente ecuación:

$$
H_{u 2 u}(t)=H_{u 2 u}(0) \cdot\left(1+C A G R_{u 2 u}\right)^{t}
$$

siendo $H_{u 2 u}(\mathrm{t})$ la matriz de tráfico User-to-user para un instante temporal $t, H_{u 2 u}(0)$, la matriz de tráfico en el año cero de la simulación y $C A G R_{u 2 u}$, la tasa de crecimiento anual para el tráfico usuario a usuario.

Para cada pareja de nodos, el tráfico se sirve gracias al tipo de encaminamiento unicast, donde a través de la ruta mas corta, se establece el flujo de tráfico entre los nodos origen y destino correspondientes a lo largo de la red de transporte. Este tráfico se supone bidireccional.

\subsubsection{Tráfico $D C$-to-user}

DC-to-user traffic representa el tráfico relacionado con los servicios en la nube, donde los usuarios cercanos a los nodos de la red de transporte reclaman el contenido de las aplicaciones que están alojadas en los datacenters de uno o varios CDNs. Normalmente el datacenter con réplica del contenido deseado mas cercano al usuario es quién se encarga de suministrar la demanda requerida (anycast routing). Cada tipo de servicio tiene asociado un CAGR particular [Cisco17], utilizado por las aplicaciones para conocer el tráfico requerido por el total de los usuarios en cada tiempo. De manera análoga al tráfico 
User-to-user, para cada instante temporal y cada aplicación, se calcula el tráfico ofrecido según el tipo de servicio que ofrezca.

$$
H_{a}(t)=H_{a}(0) \cdot\left(1+C A G R_{s}\right)^{t}
$$

siendo $H_{a}(\mathrm{t})$ el tráfico ofrecido User-to-user para un instante temporal $t$ y para una aplicación $a, H_{a}(0)$ es la matriz de tráfico en el año cero de la simulación para tal aplicación y $C A G R_{s}$, la tasa de crecimiento anual para el servicio $s$.

En contraposición con el tráfico User-to-user, donde se conocían previamente los nodos origen y destino, así como la cantidad de tráfico requerida, para el tipo anycast la demanda puede ser satisfecha por un subconjunto de posibles nodos origen para un único destino. El peso del tráfico requerido a cada nodo de la red de transporte (usuarios), no sólo es proporcional a su población, sino también es relativo al volumen de tráfico total de la aplicación y a la popularidad del contenido deseado. De tal manera que la unidad de contenido mas popular que sea reclamada por el nodo de mayor población será significativamente mayor que el volumen de tráfico deseado por un nodo de baja población reclamando un contenido poco popular. Además, el total del contenido de la aplicación es dividido en 100 partes, con el peso proporcional a la popularidad del contenido, el cual está modelado a través de una distribución normalizada de Zipf tipo $1 / f$. De tal manera que, como se muestra en la Figura 2.3, el contenido más popular es 100 veces mayor que el contenido menos popular. El tráfico total de la aplicación está dividido equitativamente entre todos los CDNs que sirven a tal aplicación. Se considera además que cada unidad de contenido tiene al menos dos réplicas en cada $\mathrm{CDN}$, el contenido original más uno de respaldo o backup.

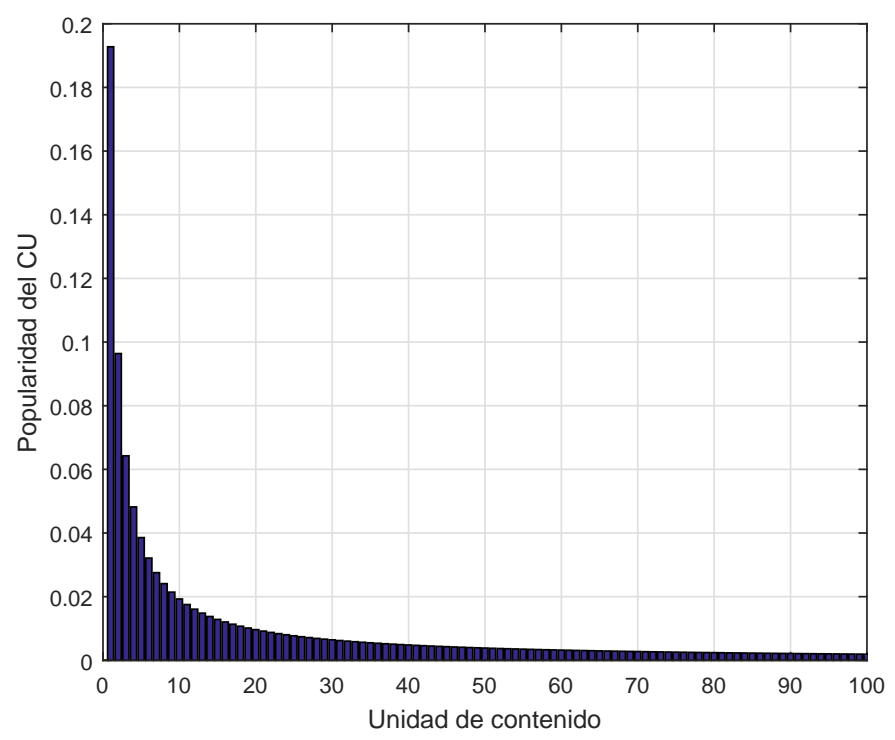

Figura 2.3: Modelado de popularidad de contenido: distribución normalizada de Zipf 1/f.

El modelo considera el conjunto de servicios dado en [Cisco16a] y [Cisco17] y es demandado a través de varias aplicaciones representativas. A pesar del hecho que en la realidad una aplicación puede servir diferentes servicios, por simplicidad, se considera 
que una aplicación ofrece el tráfico de un único servicio. El conjunto de servicios asumidos en este estudio es el siguiente: (a) long Internet video, algunas de las aplicaciones representativas pueden ser Netflix o Amazon Prime Video, (b) short Internet video, como puede ser YouTube, (c) web, email and data, que incluye además otros tipos de tráfico de datos no compartidos, (d) file sharing, aplicaciones similares a peer-to-peer (P2P) y (e) Online gaming, por ejemplo, Xbox Games o PlayStation Network.

\subsubsection{Tráfico $D C$-to- $D C$}

La migración de réplicas de CU, así como su sincronización entre los DCs que los contienen, conforman el segundo tipo de tráfico con dependencia de los servicios en la nube. El tráfico $D C$-to- $D C$ asume el paradigma de encaminamiento multicast entre los datacenters de cada red de reparto de contenido que alojan las réplicas. En el caso que el emplazamiento de las réplicas no sea conocido con anterioridad, el contexto multicast se traslada a manycast routing, donde solo un subconjunto del total de los nodos será interconectado como posibles destinos para albergar el contenido.

Como se ha expuesto anteriormente, para cada aplicación, el tráfico $D C$-to- $D C$ está íntimamente relacionado con el tráfico $D C$-to-user y con la naturaleza del servicio que ofrece dicha aplicación. El volumen total de tráfico inter- $D C$ es proporcional tanto al trafico $D C$-to-user ofrecido, como al factor $\beta$ del correspondiente servicio. A su vez, el tráfico es distribuido por un árbol multicast mínimo para cada unidad de contenido que une todos los nodos con réplica.

\subsubsection{Algoritmo de optimización para la evolución de tráfico core (MP-CTE)}

Considerando los supuestos en la red de transporte, las diferentes políticas de crecimiento propuestas y los modelos de tráfico analizados en anteriores secciones, en este apartado se presenta un método multi-periodo con el objetivo de minimizar el tráfico en la red de transporte. En la Figura 2.4 se expone el pseudocódigo del algoritmo llamado Multi-Period Core Traffic Evolution (MP-CTE), el cual forma parte del trabajo presentado en esta tesis.

Es relevante mencionar que no se asumen enlaces core con capacidad finita, sino que se considera que los propietarios de la red de transporte asumen el coste de expandir la capacidad de los enlaces para soportar satisfactoriamente el aumento de tráfico a considerar. Además, en este modelo no existe limitación de capacidad en los datacenters y todas las conexiones se asumen bidireccionales.

En primera instancia (línea 1), se calculan las rutas asociadas a el tráfico independiente de los CDNs por el camino mas corto entre los dos nodos involucrados. Nótese que, dada la capacidad en los enlaces no es una limitación, el tráfico entre usuarios no afecta a la optimización. Asumiendo $t=0$, es decir, antes de comenzar el tiempo de simulación, es necesario calcular, para cada CDN considerado, el número de réplicas de cada unidad de contenido además de su emplazamiento inicial en los datacenters disponibles. Estos objetivos se logran ejecutando un ILP (Integer Liner Program), denominado ILP-RP (línea 3), orientado especialmente para satisfacer tales propósitos, cuya función objetivo es 
minimizar el tráfico total en los enlaces core aportado por el CDN (anycast + multicast). Se aportan mayores detalles sobre esta tarea en la próxima subsección. Una vez conocido el número de réplicas así como su posición en el CDN, se procede a calcular routing tanto del tráfico $D C$-to-user como el $D C$-to- $D C$ en el instante temporal inicial (líneas 4 y 5), de esta manera, facilitando el cálculo del tráfico cursado en la red de transporte utilizando el routing calculado en los pasos anteriores (línea 8 ).

Comienzo del bucle principal. Para cada año, el tráfico ofrecido de cada tipo es aumentado en consecuencia acorde a su CAGR (líneas 12 a 15). En base al aumento de tráfico en cada CDN, se calcula, respecto del último año con un DC creado en tal CDN, si es necesario iniciar el proceso de apertura de un nuevo DC, según la política de expansión elegida (línea 16), es decir, el parámetro $G$. La ecuación que rige la decisión es la siguiente:

$$
a d d D C=G \cdot \frac{H_{c}(t)-H_{c}\left(t_{c}^{\prime}\right)}{H_{c}\left(t_{c}^{\prime}\right)}
$$

con $G$ el parámetro de expansión de $\mathrm{CDN}, H_{c}(\mathrm{t})$ el total de tráfico cursado por el CDN $c$ en el instante de tiempo $t$ y $H_{c}\left(t_{c}^{\prime}\right)$ el tráfico total en los enlaces correspondiente al CDN $c$ en el último instante en que fue creado el úlitmo DC, $t_{c}^{\prime}$. Es importante resaltar que estos procesos se realizan individualmente para cada CDN.

En caso que $a d d D C$ sea mayor o igual a 1, se asume que es necesario evaluar los candidatos disponibles para crear un nuevo DC en las proximidades de los nodos core. En este punto (líneas 20-28), entran en contexto las políticas de localización para los nuevos DCs enunciados anteriormente, TrOpt y Rand. Si se tiene en consideración TrOpt, el algoritmo ejecuta secuencialmente el ILP-RP asumiendo que existe un DC en el nodo candidato y calcula el impacto que tendría en la red. Tras analizar el rendimiento de la red para todos los candidatos, se elige como localización definitiva aquel que minimiza el aporte total de tráfico (anycast y multicast) a la red de transporte. Mientras que la estrategia de localización de DCs Rand, selecciona aleatoriamente un nodo sin DC en ese CDN como candidato para la apertura de un nuevo datacenter. Tanto para TrOpt como para Rand, se asume como DC no creado en el caso que el nuevo DC elegido se encuentre vacío de contenido tras realizar el reparto de réplicas (ILP-RP). Finalmente, para ese instante temporal $t$, se calcula el tráfico total en la red.

\subsubsection{ILP para localización de réplicas (ILP-RP)}

El algoritmo ILP-RP trata de resolver dos principales problemas de forma conjunta: (a) el número de réplicas de cada unidad de contenido para todas las aplicaciones servidas por un CDN, así como, (b) el datacenter elegido para almacenar cada réplica individualmente. La función objetivo de este ILP es minimizar el tráfico aportado por el CDN que termine fluyendo en la red core teniendo en cuenta el coste, proporcional al número de saltos del total del tráfico anycast y una estimación inferior para el coste multicast. Es importante aclarar que el routing mulitcast no aporta un valor exacto del coste en saltos del árbol multicast debido a que en esta subrutina del algoritmo MP-CTE no se calcula el enrutamiento del tráfico $D C$-to- $D C$, ese proceso lo realiza el algoritmo ILP-MC.

Los parámetros de entrada de este ILP se exponen como sigue: 


\section{Input:}

Define $\mathcal{N} \quad / /$ Conjunto de nodos en el core;

Define $\mathcal{U} / /$ Conjunto de unidades de contenido;

Define $\mathcal{A} / /$ Conjunto de aplicaciones;

Define $\mathcal{C} / /$ Conjunto de CDNs que sirven el tráfico de apps:

Define $\mathcal{D}_{c} / /$ Initial set of DCs in each CDN $c \in \mathcal{C}$;

$H_{-} u 2 u(0) \quad / /$ Tráfico ofrecido $\mathrm{u} 2 \mathrm{u}$ en el instante inicial;

$H_{a c}(0)$ / Tráfico ofrecido por la aplicación $a$ servida por CDN $c$ in el instante inicial;

$\beta_{a} \quad / /$ Factor $\beta$ de por aplicación $a$;

$C A G R \_u 2 u, C A G R_{a} \quad$ //CAGR para el tráfico u2u y para tráfico CDN por aplicación $a$;

$G \quad / / F a c t o r$ de crecimiento del CDN;

$T$ //Número de periodos temporales;

Output:

Begin:

1 Calcula $R \_u 2 u$ //Conjunto de shortest paths para enrutar $\mathrm{u} 2 \mathrm{u}$, constante $\forall t \in \mathcal{T}$;

2 foreach $c \in \mathcal{C}$ do

3 Ejecuta ILP-RP para emplazar todos los CUs $u$ servido por CDN $c$ en el tiempo inicial;

$4 \quad$ Ejecuta ILP-AC para calcular rutas anycast $R_{-} u 2 d c(c, 0)$ para CDN $c$ en el tiempo inicial;

$5 \quad$ Ejecuta ILP-MC para calcular árboles multicast $R_{-} d c 2 d c(c, 0)$ para CDN $c$ en el tiempo inicial;

6 end

$7 R(0)=\left\{R_{-} u 2 u, \cup_{c \in \mathcal{C}} R_{-} u 2 d c(c, 0), \cup_{c \in \mathcal{C}} R_{-} d c 2 d c(c, 0)\right\}$ //Solución inicial completa de enrutamiento;

8 Calcula coreTraffic(0) //Tráfico total en los enlaces basado en el tráfico ofrecido y $\mathrm{R}(0)$;

$9 t=1 / /$ Instante temporal actual;

$10 t_{c}^{\prime}=0, \forall c / /$ Último instante temporal que un nuevo DC fue añadido a $\operatorname{CDN} c$;

11 do

$12 \quad H_{\_} u 2 u(t)=H_{\_} u 2 u(0) \cdot\left(1+C A G R \_u 2 u\right)^{t} \quad / /$ Calcula tráfico u2u en instante $t$;

$13 \quad R(t)=R(t-1) / /$ Inicialmente se asume que existe solución de enrutamiento;

14 foreach $c \in \mathcal{C}$ do

$15 \quad$ Calcula $H_{c}(t) / /$ Tráfico total ofrecido por CDN $c$ en instante $t$, donde el tráfico ofrecido por app crece como $H_{a c}(\mathrm{t})=H_{a c}(0) \cdot\left(1+C A G R_{a}\right)^{t} ;$

16 Calcula coreTraffic $(c, t)$ para $\mathrm{CDN} c$ con DCs existentes y solución de routing;

$17 \quad$ add $D C=G \cdot\left(H_{c}(t)-H_{c}\left(t_{c}^{\prime}\right)\right) / H_{c}\left(t_{c}^{\prime}\right)$;

18 if $a d d D C \geq 1$ then

$19 \quad \mid$ new $D C=0$;

$20 \quad$ foreach $n \in \mathcal{N} / \mathcal{D}_{c}$ do

$21 \quad$ Ejecuta ILP-RP para emplazar todos los CUs $u$ servidos por CDN $c$ en el instante $t$ asumiendo un nuevo DC en el nodo $n$;

Ejecuta ILP-AC para calcular las rutas anycast resultantes $R \_u 2 d c(c, t, n)$;

Ejecuta ILP-MC para calcular los árboles multicast $R_{-} d c 2 d c(c, t, n)$;

Calcula coreTraffic $(\mathrm{c}, \mathrm{t}, \mathrm{n})$;

if coreTraffic $(c, t, n)<$ coreTraffic $(c, t)$ then

end $n e w D C=n$

end

if newDC $\neq 0$ then

$\mathcal{D}_{c}=\mathcal{D}_{c} \cup n e w D C ;$

Actualiza $R(t)$ con $R_{-} u 2 d c(c, t, n e w D C)$ y $R_{\_} d c 2 d c(c, t, n e w D C)$;

$t_{c}^{\prime}=t$

end

end

end

Calcula coreTraffic(t) //Tráfico total en el core en el instante $t$;

$t++$;

while $t \leq T$

39 End;

Figura 2.4: Algoritmo de optimización para la evolución de tráfico core (MP-CTE) 
- $\mathcal{U}$ : Conjunto de unidades de contenido (content units, CUs).

- $\mathcal{A}$ : Conjunto de aplicaciones servidas en un $\mathrm{CDN}$.

- $\mathcal{N}$ : Conjunto total de nodos en la red core.

- $\mathcal{D}$ : Conjunto de datacenters en un $\mathrm{CDN}$.

- $\hat{z_{u}}, u \in \mathcal{U}$ : Popularidad de un CU según la distribución normalizada de Zipf.

- $h_{a}, a \in \mathcal{A}$ : Tráfico total para una aplicación $a$.

- $\hat{p_{n}}, n \in \mathcal{N}$ : Población total servida por el nodo $n$.

- $\mathrm{c}_{n d}, n \in \mathcal{N}, d \in \mathcal{D}:$ Coste en número de saltos siguiendo la ruta mas corta entre un nodo $n$ y el DC $d$.

- $\beta_{a}, a \in \mathcal{A}$ : Factor $\beta$ de una aplicación $a$.

Mientras que las variables de decisión elegidas para almacenar las soluciones del ILP con:

- $c_{\text {uand }}, u \in \mathcal{U}, a \in \mathcal{A}, n \in \mathcal{N}, d \in \mathcal{D}$ : es 1 si para el CU $u$ en la aplicación $a$, el DC con réplica $d$ es el mas cercano al nodo $n .0$ en caso contrario.

- $r_{u a d}, u \in \mathcal{U}, a \in \mathcal{A}, d \in \mathcal{D}: 1$ si el DC $d$ contiene una réplica del CU $u$ de una aplicación $a .0$ en caso contrario.

La función objetivo es:

$$
\min \sum_{u, a, n, d} \hat{z_{u}} \cdot h_{a} \cdot \hat{p_{n}} \cdot c_{n d} \cdot c l_{u a n d}+\sum_{u, a, d} \hat{z_{u}} \cdot h_{a} \cdot \beta_{a} \cdot\left(\sum_{u, a, d} r_{u a d}-1\right)
$$

La ecuación (2.4) minimiza el tráfico total en la red core aportado por el CDN compensando el tráfico anycast y el relativo a multicast. La parte de la izquierda satisface las exigencias de los usuarios, mientras que la parte derecha realiza una estimación inferior del coste en sincronización en el reparto de contenido. Nótese que el mínimo número de saltos en un árbol multicast entre los DCs con réplica de un CU es el número de réplicas menos 1 .

Las restricciones consideradas para ILP-RP se presentan como:

$$
\begin{gathered}
\sum_{d \in \mathcal{D}} c l_{\text {unad }}=1 \quad \forall u \in \mathcal{U}, a \in \mathcal{A}, n \in \mathcal{N} \\
\sum_{d \in \mathcal{D}} r_{\text {uad }} \geq 2 \quad \forall u \in \mathcal{U}, a \in \mathcal{A} \\
\sum_{u \in \mathcal{U}} \sum_{a \in \mathcal{A}} \sum_{d \in \mathcal{D}} c l_{\text {uand }} \leq r_{\text {uad }} \quad \forall n \in \mathcal{N}
\end{gathered}
$$


Por otra parte, la ecuación (2.5) asegura que únicamente exista un DC más cercano a cada nodo, para cada aplicación y CU. El requisito de cumplimiento de que cada CU tenga al menos dos réplicas se presenta en (2.6). Finalmente, (2.7) restringe que en ningún momento el número total de DCs más cercanos a un nodo $n$ sea mayor que el número total de réplicas de una unidad de contenido.

\subsection{Resultados numéricos}

En la presente sección se exponen y analizan los resultados de los estudios realizados bajo el contexto de evolución temporal del tráfico IP en dos redes core de referencia, así como un análisis $\beta$-paramétrico que analiza el impacto de la naturaleza de los servicios en las redes de transporte a lo largo del tiempo. Ambos estudios se han realizado utilizando como contexto la herramienta de planificación de redes Net2plan [Net2P] $[$ Pavon15], la cual incluye, JOM (Java Opitimzer Modeller) una interfaz de comunicación con el solver IBM CPLEX para dar salida a las soluciones del algoritmo ILP-RP.

\subsubsection{Evolución de tráfico core bajo crecimiento de CDNs}

\subsubsection{Escenario de simulación}

Las simulaciones del estudio temporal de tráfico asumiendo crecimiento en las redes de reparto de contenido han sido realizadas en dos topologías de referencia que emulan la red de transporte core, NSFNet, con 14 nodos y una distancia promedio entre cada pareja de nodos de la red de 2.14 saltos, y la European Optical Network (EON), con 18 nodos y una distancia promedio nodo a nodo de 2.24 saltos. Ambas redes están disponibles en $[\overline{\mathrm{Net}} 2 \mathrm{P}]$. El tráfico core considerado en este estudio se corresponde con las directrices expuestas en la sección anterior, con un peso total para cada tipo de tráfico entre usuarios acorde con [Cisco17], es decir, no dependiente de CDNs, conformando un $24 \%$ del total, mientras que el tráfico demandado por los usuarios de la red core a los CDNs, el $76 \%$ restante. Se considera el tráfico ofrecido total normalizado a uno en el año inicial. Por otra parte, el volumen de tráfico inter-DC es obtenido según el factor $\beta$ de las aplicaciones (servicios) que se consideran.

El tráfico basado en cloud reclamado por los usuarios es servido por 20 aplicaciones diferentes, que a su vez ofrecen el contenido de 5 servicios distintos. Con el fin de simplificar, se asume únicamente un servicio por cada aplicación. Los servicios tienen asociados valores fijos de la tasa de crecimiento anual, CAGR, únicos para cada servicio considerado, obtenidos de [Cisco17]. El peso respecto del porcentaje total de tráfico para cada tipo de servicio ha sido calculado en consecuencia gracias a [Cisco17] y [Sandv16] de tal manera que la suma de todos los pesos hace un valor del $76 \%$ del tráfico usuario total. Además, todas las aplicaciones que ofrecen un determinado servicio comparten el mismo valor de $\beta$. El conjunto de valores de $\beta$ ha sido seleccionado arbitrariamente para emular la naturaleza de cada servicio. Un resumen de los servicios se presenta en la Tabla 2.1. Se asume un total de 5 CDNs en las simulaciones que soportan el tráfico basado en la nube, donde el tráfico de cada aplicación puede ser servido por 1 a 3 CDNs. 
Tabla 2.1: Lista de servicios de Internet

\begin{tabular}{|c|c|c|c|c|}
\hline Servicios & Aplicación & Tráfico Usuario & CAGR & $\beta$ \\
\hline Vídeo (largo) & Netflix & 0.465 & $31 \%$ & 0.05 \\
\hline Vídeo (corto) & YouTube & 0.252 & $31 \%$ & 0.25 \\
\hline Web, email y datos & Instagram & 0.154 & $17 \%$ & 0.5 \\
\hline Compartición de archivos & Bit Torrent & 0.113 & $0 \%$ & 0.45 \\
\hline Juegos en línea & PlayStation Store & 0.013 & $62 \%$ & 0.05 \\
\hline
\end{tabular}

Respecto a las políticas de crecimiento de CDN, para este estudio se valoran tres políticas distintas, $G=0,1,2$, es decir, para $G=0$, no se crean nunca nuevos DCs, mientras que cuando $G$ vale 1 y 2 , se plantea la ampliación del CDN al aumentar el tráfico en el CDN un $100 \%$ y un $50 \%$ respectivamente, desde el último año con una nueva apertura de un datacenter. Para estos últimos dos valores de $G$ se emplean las dos estrategias de localización de DCs explicadas para el modelo, TrOpt y Rand. Las ejecuciones para resolver el algoritmo ILP-RP individualmente han sido limitadas a 60 segundos cada una para obtener el óptimo, transcurrido este tiempo se devuelve la mejor solución subóptima. Este último caso se ha encontrado como muy residual siendo encontrado el resultado óptimo en la amplia mayoría de las simulaciones ejecutadas.

\subsubsection{Resultados}

El tráfico total normalizado tras un intervalo de 15 años para las topologías NSFNet y EON se muestran en las figuras 2.5 (a) y 2.5 (b), respectivamente, expresadas en una escala semi-logarítmica en el eje vertical. Para ambas topologías, se aprecia como el tráfico total en los enlaces ha disminuido considerablemente para $G=1$ y 2 , es decir, cuando se crean nuevos datacenters respecto de la opción en la que todo el tráfico viene soportado por los DCs originales $(G=0)$. Nótese que la reducción es especialmente significativa para la estrategia de localización de DCs TrOpt, este hecho se cataloga como esperable, ya que, la propia elección de dónde abrir el $D C$ está optimizada de base. Observando ambas representaciones gráficas en Fig. 2.5 puede visualizarse una marcada zona donde las curvas para $G=1$ y 2 se separan de la tendencia de $G=0$ en forma de sus respectivas curvas. Tal suceso sucede entre los años 2 y 8 aproximadamente, siendo para la topología NSFNet especialmente marcada dada la naturaleza de su grafo, ya que tiene un número de saltos promedio menor, donde no solo se reduce considerablemente la tasa de crecimiento anual de tráfico, sino también incluso puede bajar el volumen de tráfico en los enlaces para los primeros años de simulación. Este suceso viene justificado por el hecho de que la creación de un nuevo DC tiene mayor impacto en el tráfico en la red cuando existen pocos DCs en el CDN respecto de los instantes temporales en los que el usuario ya tiene, relativamente cerca, el contenido. Es decir, se puede afirmar que el beneficio de la apertura de un nuevo datacenter será mayor cuanto menor sea el tamaño del CDN en número de DCs. Especialmente relevante el caso TrOpt en el cual la localización del primer DC elegido es precisamente el que mayor reducción tiene en el tráfico de los enlaces core, tal hipótesis está sustentada por los resultados obtenidos.

Para dar mas valor los resultados, la Tabla 2.2 resume los valores estimados para el 


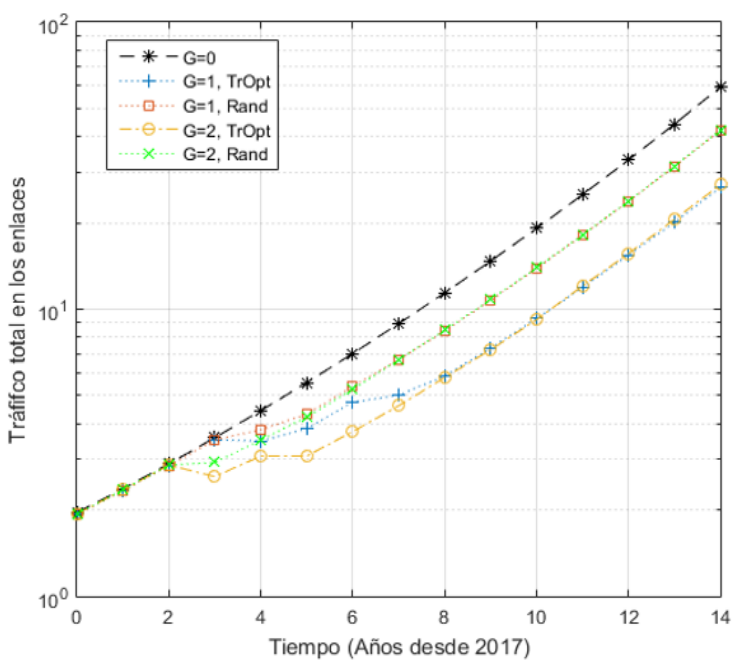

(a)

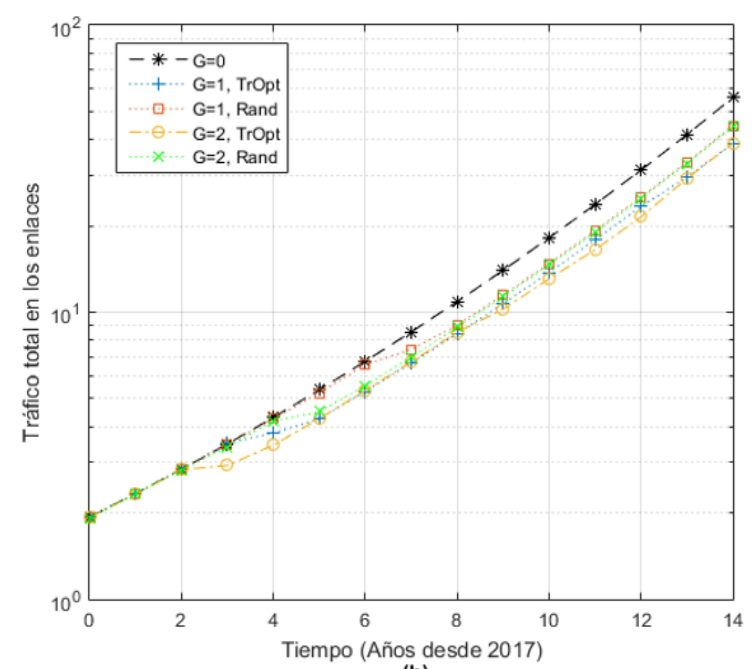

(b)

Figura 2.5: Tráfico total en los enlaces core para las topologías (a) NSFNet y (b) EON, durante 15 años.

crecimiento de tráfico anual (CAGR) para las ejecuciones de cada topología y estrategia de creación de DC, no solo para el tráfico total, si no también desglosado en tipo de tráfico, de esta manera se puede realizar una mejor comprensión del impacto temporal de cada tipo de tráfico en la red core, los valores entre paréntesis representan los valores normalizados totales de tráfico tras los 15 años de ejecución, recuérdese que en el año 0 este valor se corresponde con 1 para el tráfico ofrecido, por lo que para tales valores de la Tabla 2.2 muestran una directa multiplicidad en cantidad total de tráfico respecto del año inicial. Un hecho que soporta los resultados es que, tanto para las ejecuciones en NSFNet y en EON, la tendencia de crecimiento de tráfico total cuando no se crean DCs es de $27,4 \%$ para NSFNet y 27,2\% en EON, completamente en línea con las previsiones de crecimiento del informe Cisco VNI [Cisco17] que marcan un valor del $27 \%$ anual.

Por otro lado, el análisis de tráfico para los valores $G=1$ y 2 , muestra un claro descenso en el crecimiento de anual de tráfico para todos los escenarios. Se puede apreciar que para la política de localización de DCs, Rand, y $G=1$, para el caso NSFNet ha experimentado una disminución en el CAGR total desde el $27.4 \%$ hasta el $23.6 \%$, mientras que aplicado a EON, ha pasado desde un un $27.2 \%$ a un $24.4 \%$. Tal reducción en el CAGR implica una disminución en el tráfico total del $29 \%$ y el $20 \%$ respectivamente. Para los mismos escenarios aplicando TrOpt, la optimización consigue mejorar el rendimiento hasta obtener unos valores de $19.5 \%$ en NSFNet (56\% menos de tráfico) y $23.2 \%$ ( $36 \%$ menor en volumen de tráfico) en EON comparado con $G=0$. Especialmente interesante es el hecho que no varían significativamente tales resultados para la aplicación de $G$ = 2. La Figura 2.5 deja en evidencia que el beneficio de la apertura de DCs es evidente, en similitud de escenarios, la única diferencia sustancial entre $G=1$ y $G=2$, es el que tal beneficio se obtendrá antes si se abren mas DC, pero en cualquiera de los dos casos el beneficio final será similar tras 15 periodos. Existe un punto en el tiempo, en el que para este escenario de simulaciones, abrir nuevos datacenters no mejora la situación de tráfico en los enlaces. 
Tabla 2.2: CAGR total del tráfico (y trafico cursado) para un periodo de 15 años.

\begin{tabular}{|c|c|c|c|c|c|c|c|c|c|c|}
\hline \multirow[b]{3}{*}{ Red } & \multirow{2}{*}{\multicolumn{2}{|c|}{$G=0$}} & \multicolumn{4}{|c|}{$G=1$} & \multicolumn{4}{|c|}{$G=2$} \\
\hline & & & \multicolumn{2}{|c|}{ Rand } & \multicolumn{2}{|c|}{ TrOpt } & \multicolumn{2}{|c|}{ Rand } & \multicolumn{2}{|c|}{ TrOpt } \\
\hline & NSFNet & EON & NSFNet & EON & NSFNet & EON & NSFNet & EON & NSFNet & EON \\
\hline $\begin{array}{c}\text { Total trafico } \\
\text { core }\end{array}$ & $\begin{array}{l}27.4 \% \\
(59.4) \\
\end{array}$ & $\begin{array}{l}27.2 \% \\
(55.9)\end{array}$ & $\begin{array}{c}23.6 \% \\
(42.2)\end{array}$ & $\begin{array}{l}24.4 \% \\
(44.6)\end{array}$ & $\begin{array}{l}19.5 \% \\
(26.5)\end{array}$ & $\begin{array}{l}23.2 \% \\
(38.7)\end{array}$ & $\begin{array}{c}23.6 \% \\
(42.5) \\
\end{array}$ & $\begin{array}{c}24.3 \% \\
(44.2)\end{array}$ & $\begin{array}{l}19.5 \% \\
(27.1) \\
\end{array}$ & $\begin{array}{r}23.0 \% \\
(38.1) \\
\end{array}$ \\
\hline $\begin{array}{c}\text { Tráfico } \\
\text { User-to-user }\end{array}$ & $\begin{array}{l}14 \% \\
(2.3)\end{array}$ & $\begin{array}{l}14 \% \\
(2.7)\end{array}$ & $\begin{array}{l}14 \% \\
(2.3)\end{array}$ & $\begin{array}{l}14 \% \\
(2.7)\end{array}$ & $\begin{array}{l}14 \% \\
(2.3)\end{array}$ & $\begin{array}{l}14 \% \\
(2.7)\end{array}$ & $\begin{array}{l}14 \% \\
(2.3)\end{array}$ & $\begin{array}{l}14 \% \\
(2.7)\end{array}$ & $\begin{array}{l}14 \% \\
(2.3)\end{array}$ & $\begin{array}{l}14 \% \\
(2.7)\end{array}$ \\
\hline $\begin{array}{c}\text { Tráfico } \\
\text { DC-to-user }\end{array}$ & $\begin{array}{l}30.5 \% \\
(46.0)\end{array}$ & $\begin{array}{l}30.8 \% \\
(41.1) \\
\end{array}$ & $\begin{array}{l}25.3 \% \\
(27.0) \\
\end{array}$ & $\begin{array}{l}27.9 \% \\
(31.1) \\
\end{array}$ & $\begin{array}{l}18.1 \% \\
(14.0)\end{array}$ & $\begin{array}{l}23.9 \% \\
(22.0) \\
\end{array}$ & $\begin{array}{l}25.2 \% \\
(26.8) \\
\end{array}$ & $\begin{array}{l}26.6 \% \\
(30.8) \\
\end{array}$ & $\begin{array}{l}16.7 \% \\
(12.3) \\
\end{array}$ & $\begin{array}{l}22.9 \% \\
(20.3) \\
\end{array}$ \\
\hline $\begin{array}{c}\text { Tráfico } \\
D C \text { - } t o-D C\end{array}$ & $\begin{array}{l}24.9 \% \\
(10.9)\end{array}$ & $\begin{array}{l}24.9 \% \\
(12.1)\end{array}$ & $\begin{array}{l}28.2 \% \\
(12.4)\end{array}$ & $\begin{array}{l}23.6 \% \\
(10.8)\end{array}$ & $\begin{array}{l}26.4 \% \\
(11.2)\end{array}$ & $\begin{array}{l}28.4 \% \\
(14.0)\end{array}$ & $\begin{array}{l}28.4 \% \\
(12.5)\end{array}$ & $\begin{array}{l}23.6 \% \\
(10.8)\end{array}$ & $\begin{array}{l}28.4 \% \\
(12.5)\end{array}$ & $\begin{array}{l}29.4 \% \\
(15.8)\end{array}$ \\
\hline
\end{tabular}

La Tabla 2.2 muestra además información sobre el impacto obtenido para cada configuración en el tráfico y su crecimiento según cada tipo de tráfico considerado. Como era esperable, el tráfico User-to-user, no sufre cambio alguno en el proceso de expansión de CDNs, hecho evidente debido a su independencia con este tipo de redes. Respecto del DC-to-user traffic, se ha producido la mayor reducción en el CAGR de los tres tipos de tráfico, acercar el contenido al usuario implica menor tráfico en los enlaces gracias un menor número de saltos en las rutas para satisfacer las demandas. Tal reducción de tráfico de este tipo respecto de $G=0$ ha llegado hasta valores totales desde un 46.0 hasta 12.3 según $G=2$ y para NSFNet, supone que un total del $73 \%$ de tráfico respecto de $G=0$ para este tipo. En contraposición al tráfico $D C$-to-user, el tráfico asociado a los procesos de actualización y sincronización ha sido mayor conforme crece el tamaño de los CDNs. El escenario considerado donde se aprecia el mayor aumento dentro del marco supuesto ha sido en la topología EON (al tener EON un grafo tipo malla y un mayor promedio de saltos entre cada pareja de nodos, el árbol multicast es mayor en número de saltos que para NSFNet) según el criterio $\operatorname{Tr} O p t$ y $G=2$. Bajo tales condiciones, el aumento total de tráfico ha sido del $31 \%$ respecto de la opción sin creación de DCs.

En esta sección se ha evidenciado que el aumento o disminución de tráfico en los enlaces core depende, en gran parte, del balance entre el tráfico tipo cloud de usuario y de sincronización, este hecho tiene dependencia directa con la naturaleza de las aplicaciones y servicios que ofrecen los distintos CDNs. Bajo esta capa, se anticipa la necesidad de realizar un estudio paramétrico que ponga de manifiesto la implicación de los servicios según el tipo que añada una nueva visión que ayude a anticipar respuestas a la incógnita sobre la evolución del tráfico en la redes de transporte.

\subsubsection{Análisis paramétrico de servicios}

Evidenciada la implicación de la naturaleza de los servicios en la congestión de las redes troncales y en la expansión de los CDNs, en esta parte del trabajo se presenta un análisis paramétrico multi-periódo para servicios individuales con diferentes valores del parámetro $\beta$, asumiendo condiciones de crecimiento de CDN fijas. Para tal conjunto de simulaciones, se han considerado cuatro aplicaciones servidas por 5 CDNs en la red NSFNet. Se asumen ejecuciones que sirven únicamente un solo tipo de servicio para cada simulación, donde todas las aplicaciones consideradas ofrecen el mismo servicio y un 
crecimiento anual de tráfico (CAGR) fijo del $30 \%$. Se han elegido cuatro valores diferentes para modelar la naturaleza de los servicios, $\beta$ igual a $0.05,0.1,0.125$ y 0.5 . Se considera un escenario tipo greenfield, donde se eligen como iniciales los dos DC con la localización mas óptima respecto al impacto en el core. Mientras que para la política de crecimiento de CDN se asume $G=2$ y según emplazamiento óptimo para los nuevos $D C s, \operatorname{TrOpt}$. En este estudio solamente se considera tráfico asociado a la nube, es decir, el tráfico User-to-user queda fuera de este escenario.

Los resultados de este experimento conceptual quedan plasmados en las figuras 2.6 (a) y (b). La Figura 2.6(a) muestra el tráfico total en los enlaces de la red de transporte en unidades arbitrarias, según los distintos servicios a lo largo del tiempo. En la parte (b) de la imagen 2.6, se considera la evolución temporal del promedio del número de DCs existentes en los CDNs para cada tipo de servicio. Se aprecia como para aplicaciones con un bajo $\beta$, representando servicios tipo Netflix que no tienen un gran tráfico inter-DC respecto del tipo usuario, se ven claramente beneficiados con el crecimiento en número de DCs de los CDN, siendo la reducción en el CAGR para el tráfico de los enlaces mas evidente en este tipo de servicio. Una disminución de tráfico en el tiempo también se aprecia para los servicios de valores $\beta=0.1$ y 0.125 . Concretamente, para el periodo de estimado de 15 años, la correspondencia servicio-CAGR ha expuesto unos resultados de CAGR $18.9 \%, 22 \%$ y $22.6 \%$ para servicios de $\beta=0.05,0.1$ y 0.125 . Estos resultados pueden ayudar a los propietarios de los CDNs a plantearse la apertura de nuevos DCs como forma de optimizar la gestión de recursos en la red transporte.

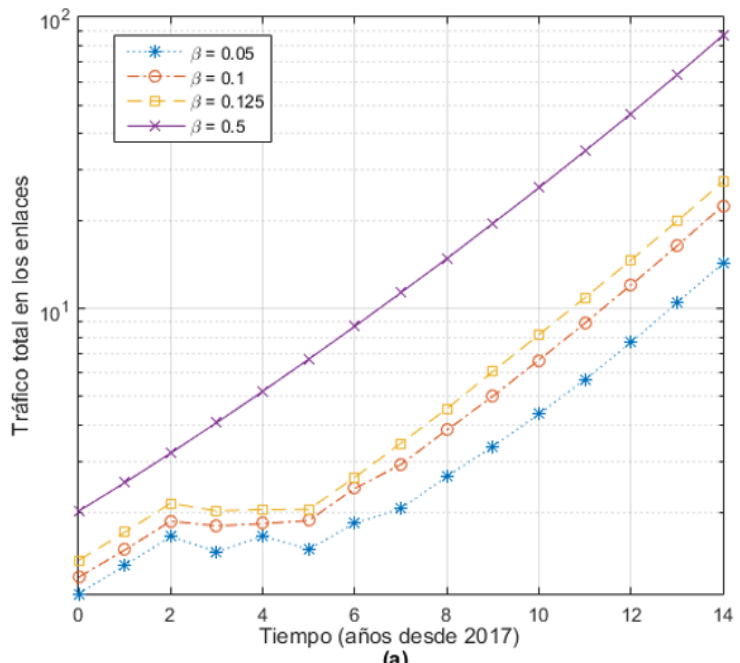

(a)

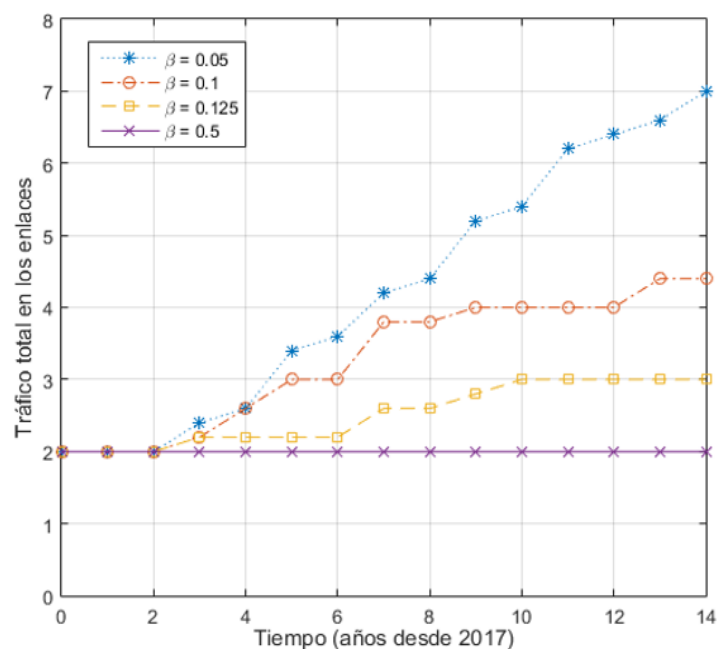

(b)

Figura 2.6: (a) Tráfico total cursado en los enlaces core y (b) número total de DCs por CDN para distintas aplicaciones.

Por el contrario, en el escenario que asume aplicaciones que ofrecen servicios con $\beta=$ 0.5 no se aprecia disminución de tráfico alguna en los enlaces core. Para este tipo de servicios donde el tráfico inter-DC es considerable, la optimización da como resultado que la compensación anycast/multicast está desbalanceada hacia el lado del coste de sincronización, por lo que el algoritmo ILP-MP no propone abrir nuevos datacenters. En este caso, se asume que los datacenters existentes soportan todo el crecimiento de tráfico. Este 
entorno puede ser beneficioso para aquellos propietarios de CDNs que se hagan cargo del tráfico de aplicaciones con una alta tasa de sincronización, en tal caso, ampliar los DC ya existentes puede ser una buena estrategia desde el punto de vista de la planificación de la red a largo plazo con objetivo de minimizar la carga en el core. En cualquier caso, los resultados en su conjunto apuntan a que las decisiones de expansión de CDNs deberían considerar la naturaleza de los servicios que ofrecen para realizar una correcta estrategia de diseño de red.

\subsection{Conclusiones}

En este capítulo se ha realizado una evaluación de la posible evolución del tráfico en las grandes redes troncales de transporte bajo circunstancias de expansión de redes de distribución de contenido. Se ha propuesto una aproximación multi-periodo para emular el comportamiento del tráfico core para un periodo de tiempo de 15 años, teniendo en cuenta diferentes políticas de expansión de $\mathrm{CDN}$, diferentes criterios de localización de nuevos datacenters, así como teniendo en consideración aplicaciones y servicios de distinta naturaleza. Además se ha complementado el trabajo con un estudio paramétrico que modela la naturaleza de los servicios según el balance de tráfico anycast/multicast. Los resultados arrojan un conjunto de mensajes o conclusiones expuestos a continuación:

- La tendencia de crecimiento del tráfico core para los próximos años tiene una estrecha dependencia con la naturaleza de los servicios soportados por los CDNs.

- El balance total entre la disminución del tráfico CDN de usuario y el aumento del tráfico de sincronización para el conjunto de servicios analizado y para un intervalo de temporal de 15 años, ha mostrado un resultado neto de reducción de tendencia de crecimiento anual para el tráfico en los enlaces core entre el $19,5 \%$ y el $23 \%$, respecto del $27 \%$ actual.

- Añadir nuevos datacenters distribuidos geográficamente puede reducir sensiblemente el tráfico soportado por las redes de transporte, la cuantía de tal reducción está íntimamente ligada a que servicios existan en el periodo de tiempo considerado.

- Planificar correctamente la apertura de nuevos datacenters en un CDN puede reducir notablemente la carga de tráfico aportada a las redes de trasporte en comparación con localizaciones aleatorias o influenciadas por otros factores de decisión.

- Una gran distribución en el contenido de servicios con bajo tráfico de sincronización respecto del tráfico servido al usuario puede aportar beneficios a los propietarios del correspondiente $\mathrm{CDN}$, en términos de carga del tráfico en los enlaces de las redes troncales.

- La ampliación en capacidad de un conjunto de pocos DCs existentes previamente puede ser una estrategia a considerar para los propietarios de CDNs que ofrezcan servicios con una alta tasa de tráfico de sincronización respecto del tráfico a usuario. 



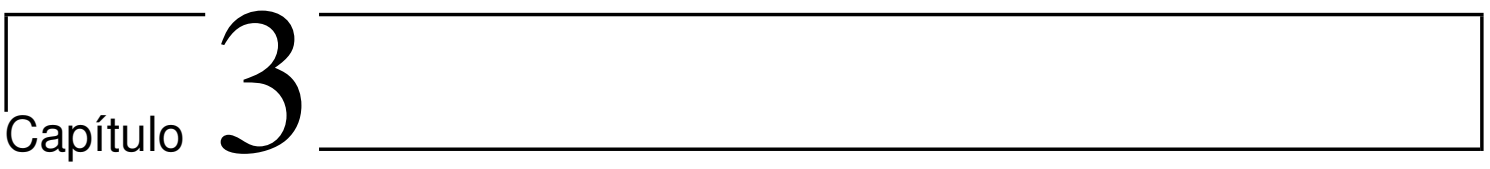

\section{Gestión y Optimización de Redes SDN/NFV}

3.1. Principios de SDN y NFV $\ldots \ldots \ldots \ldots \ldots \ldots \ldots \ldots \ldots \ldots \ldots$

3.2. Motivación de uso y herramientas open source . . . . . . . . . . . 36

3.3. Optimización en entornos SDN-NFV . . . . . . . . . . . . . . 39

3.4. Gestión en SDN-NFV. Pruebas de concepto . . . . . . . . . . . . . . . 44

3.5. Conclusiones y trabajos futuros $\ldots \ldots \ldots \ldots \ldots$

El rápido crecimiento del tráfico IP anunciado para los próximos años [Cisco17] y analizando en detalle el capítulo 2 de la presente tesis, se pueden asumir como amenazados los procedimientos tradicionales de gestión y control de redes de comunicaciones. A tal desafío se le une la necesidad de cumplimiento de las especificaciones de rendimiento para las futuras redes 5G, donde está previsto obtener, (a) un entorno de gestión escalable que permita un despliegue dinámico de aplicaciones basadas en la nube, (b) una reducción de aproximadamente un $20 \%$ en los costes operacionales y (c) una latencia total hasta el usuario menor a $1 \mathrm{~ms}$ [5Gkpi]. Tales objetivos prevén cambios sustanciales en la forma de controlar y gestionar los recursos de las redes de transporte, especialmente las redes metropolitanas.

Bajo este preocupante entorno, SDN y NFV emergen como tecnologías para poder afrontar los desafíos anteriormente expuestos de forma eficiente. En este capítulo se pone en valor el uso de entornos SDN-NFV para la correcta gestión de redes en escenarios futuros de 5G. Para tal cometido, se hace especial hincapié en el papel de la optimización en las redes de transporte gestionadas y controladas según el paradigma SDN-NFV, además de presentar los resultados y conclusiones obtenidas tras realizar pruebas de concepto en entornos de simulación realistas gestionado por herramientas open-source, orientadas a la implementación en producción para SDN y NFV.

La realización de este capítulo se ha basado en los trabajos publicados en la revista especializada IEEE Journal on Lightwave Technology [Garri18a] y en los trabajos presentados en los congresos internacionales OFC18, Los Ángeles (EEUU) [Pavon18] (Tutorial), [Moren18b] (Demo) y ECOC18 Roma (Italia) [Moren18d] (Demo). 


\subsection{Principios de SDN y NFV}

Las redes definidas por software o SDN según sus siglas en inglés, es una tecnología emergente que engloba un conjunto de técnicas orientadas a la gestión de redes que se centra principalmente en un principio básico: desacoplar las acciones de lógicas del plano de control respecto del plano de datos [OpenN12]. Este principio permite tener un alto grado de flexibilidad en el sistema, debido a que los switches de la red se convierten en simples dispositivos que envían y reciben tráfico, mientras que el control lógico de la red puede fácilmente centralizarse en un controlador externo [Kreut15]. Por otra parte, la virtualización de funciones de red o NFV, virtualiza funciones de red tradicionales, implementadas en equipamiento físico, en piezas de software que puedan ser ejecutadas en servidores. Tales funciones de red virtualizadas (VNFs) pueden ser rápidamente puestas en funcionamiento con total dinamicidad, permitiendo gran flexibilidad y escalado dentro de los datacenters de la red [Han15].

En esta sección se enuncian las principales características de las arquitecturas SDN y NFV como entes individuales, además de un análisis genérico de la dependencia y relación mutua de ambos paradigmas.

\subsubsection{Arquitectura de SDN}

Un sistema SDN presenta tres planos bien diferenciados: la capa de aplicación, la capa de control y la capa de datos. Según [Kreut15], una correcta arquitectura SDN está sustentada por cuatro pilares principales:

1. Los planos de control y datos están perfectamente desacoplados. La funcionalidad de control es eliminada de los dispositivos de red pasando a ser simples dispositivos que reciben y envían paquetes de tráfico.

2. Las decisiones de envío de tráfico están basadas únicamente en el flujo, en lugar del destino. Desde el punto de vista del contexto SDN, un flujo es una secuencia de paquetes entre un origen y un destino.

3. La lógica de control es trasladada a una entidad externa que pueda ser cómodamente ejecutada en servidores para facilitar su programación.

4. La red ha de ser programable en el sentido de que aplicaciones de software puedan ser demandadas sobre el plano de control y ejecutadas en el plano de datos.

La Figura 3.1 ilustra tales máximas de las redes SDN, el desacoplamiento entre la red de control y la capa de infraestructura (o plano de datos), con plano de aplicación en el nivel de abstracción mas alto, que se comunica con la capa de control a través de una interfaz bien definida. La inteligencia del sistema SDN está centralizada en un controlador SDN (o varios replicados como respaldo) que controla(n) y gestiona(n) de manera eficiente los recursos de la capa de datos.

Para un mejor entendimiento del paradigma SDN, a continuación se exponen los principales agentes involucrados en tal entorno: 


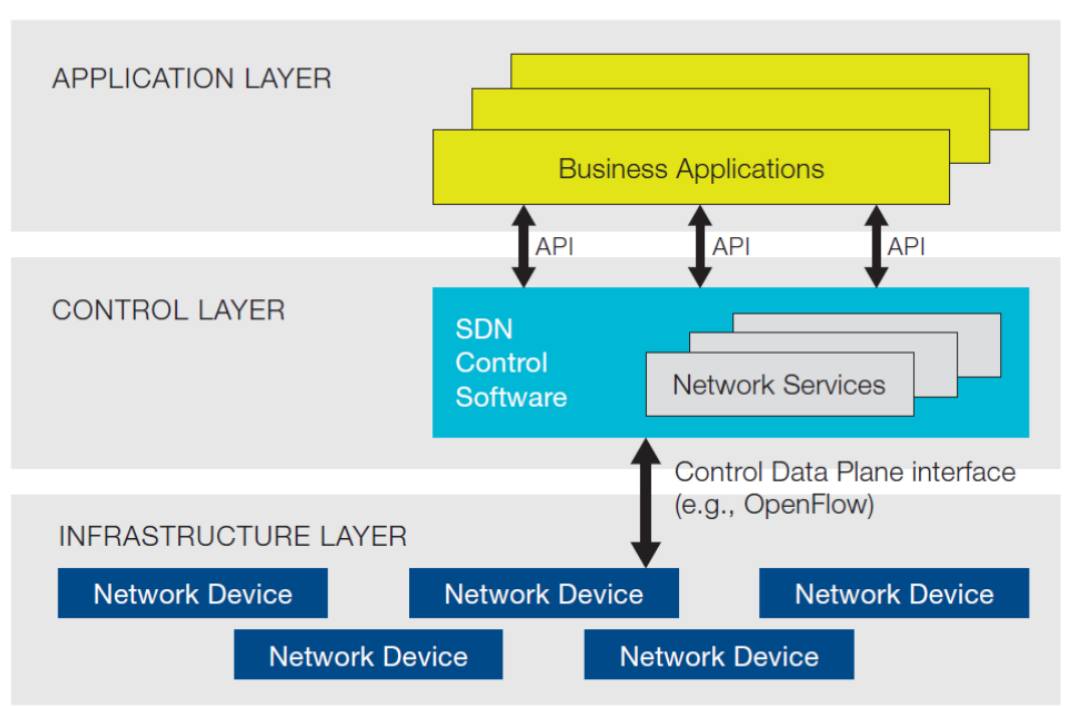

Figura 3.1: Arquitectura lógica de una red definida por software (SDN). Fuente: [OpenN12].

- Plano de aplicación: conforma un conjunto de aplicaciones que se nutren del sistema SDN para dar servicio al contenido o servicios que ofrecen tales aplicaciones. Como se aprecia en la Figura 3.1, la comunicación entre la capa de aplicación y el plano de control se realiza gracias a distintas interfaces de programación de aplicaciones (Application Programming Interfaces, APIs), donde el conjunto de todas las APIs existentes conforman la llamada interfaz norte (North Bound Interface, NBI). Un ejemplo de estándar de API para un controlador SDN de transporte es el llamado Transport API (TAPI) [OpenN17].

- Plano de control: Según el modelo de SDN de la Open Networking Foundation [OpenN12], el órgano central del plano de control es el controlador SDN, un elemento lógico de software encargado, en primera instancia, de traducir los requisitos provenientes de las aplicaciones a través de la NBI, para aplicar los cambios necesarios en los dispositivos la red de datos que satisfagan las necesidades al plano de datos. Además del controlador lógico principal, pueden coexistir varios controladores en contextos jerárquicos de varios dominios o como backups del controlador principal para aportar robustez al sistema. Por otra parte, la comunicación entre el plano de datos y el plano de control se realiza mediante la interfaz sur (South Bound Interface, $\mathrm{SBI})$.

- Plano de datos: engloba todo el conjunto de dispositivos físicos encargados de realizar el trasporte de datos en una red controlada según SDN. La gestión y configuración de estos recursos de red es llevada a cabo por el controlador SDN [OpenN13]. Como se mencionaba anteriormente, la interfaz de comunicación entre el plano de datos y el de control es la SBI. Algunas propuestas relevantes de protocolos propios de la interfaz sur son NETCONF [Ietf06] y OpenFlow [OpenN13]. 


\subsubsection{Arquitectura de NFV}

Según la ETSI [Etsi14], una arquitectura típica de un entorno NFV está compuesto por tres elementos esenciales: el orquestrador NFV, NFV Orchestrator (NFVO), también referido como NFV MANO (NFV MANagement and Orchestrator), la infraestructura NFV o NFV Infrastructure (NFVI) y finalmente el conjunto de funciones de red virtualizadas o VNFs. Este esquema se resume en la Figura 3.2 y se expone más en detalle a continuación.

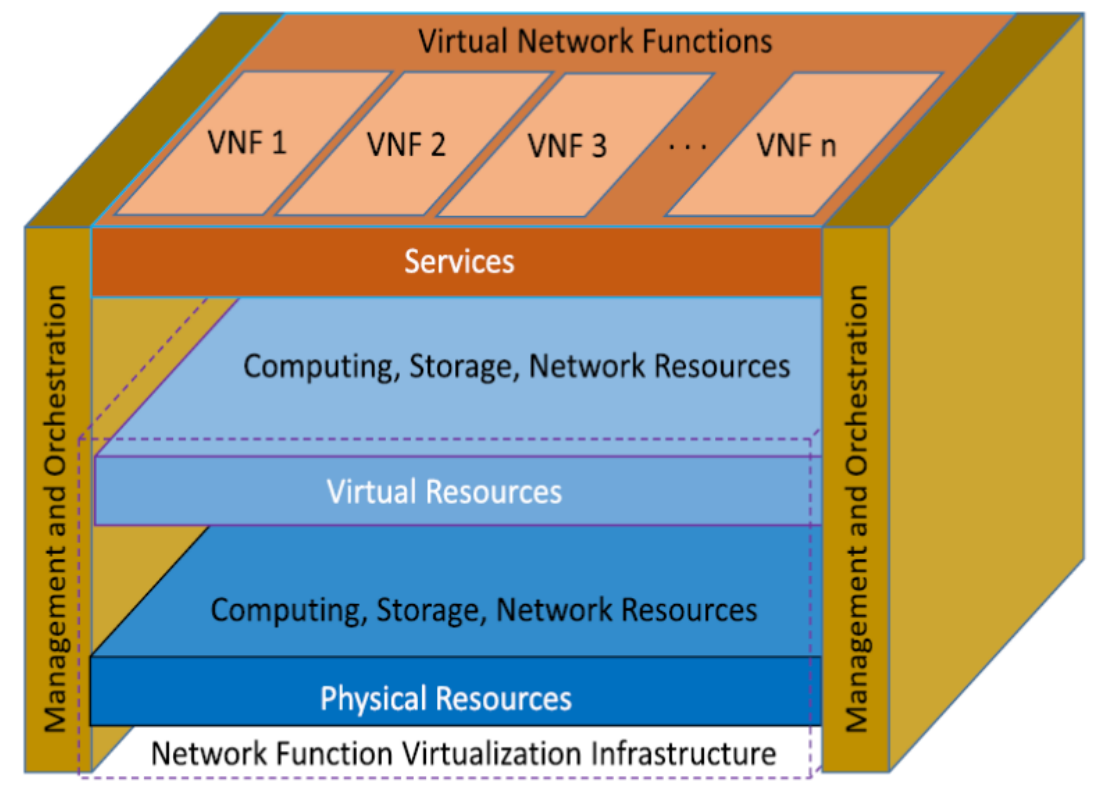

Figura 3.2: Arquitectura lógica de NFV. Fuente: [Mijum16].

- NFV MANO: según las directrices de la ETSI, el NFV MANO es aquel elemento funcional en un sistema NFV que proporciona la funcionalidad necesaria para el correcto aprovisionamiento de VNFs, así como el conjunto de todas las operaciones necesarias relativas a la virtualización, como, gestión de recursos virtuales consumidos por la VNF, configuración de la propia VNF, ciclo de vida, etc. [Etsi14][Mijum16]. Representa el elemento central en la gestión de recursos en un entorno de virtualización. También es el encargado de recibir los requisitos y especificaciones requeridos por el OSS (Operational Support System) proveniente del nivel superior (aplicación).

- NFVI: representa el conjunto de recursos tanto de hardware como de software necesario para dar soporte a la virtualización, como puede ser, la capacidad de procesamiento, memoria, red o almacenamiento. Los recursos virtuales se pueden suponer como una abstracción de tales recursos físicos. Dentro del propio NFVI, el VIM (Virtual Infrastructure Manager, es responsable de la instanciación y alojamiento de las máquinas virtuales (VMs) asociadas a las VNFs [Moren18d].

- VNF: las funciones de red virtualizadas son los elementos virtualizados mínimos con funcionalidad de red en un sistema NFV. Representan modelos virtualizados 
de funciones de red tradicionales realizadas por hardware, como por ejemplo, firewall, analizador de paquetes o NAT (Network Address Translation). Las VNFs son gestionadas por el NFV MANO para ser instanciadas en los VIMs como máquinas virtuales dotadas de la funcionalidad descrita.

\subsubsection{Combinación de SDN y NFV}

SDN y NFV conforman dos tecnologías completamente complementarias que aportan altos niveles de flexibilidad gracias a su naturaleza programable. De hecho, la combinación de SDN y NFV habilita niveles de control de red y dinamicidad sin precedentes [Garri18a], abriendo la puerta a gran cantidad de nuevas funcionalidades debido a la comunicación con la capa de aplicación. Este proceso simbiótico entre SDN y NFV es totalmente esencial para el cumplimiento de las expectativas anunciadas por el futuro paradigma 5G [5Gkpi].

Dentro de las posibles aplicaciones, quizás una de las aplicaciones en un entorno común de SDN y NFV mas relevantes sea la referente a la satisfacción de las llamadas service chains o cadenas de servicios. Una service chain es una cadena ordenada de funciones de red (VNFs) asociada a una determinada ruta en la red de transporte y está orientada para satisfacer las demandas de tráfico de un determinado servicio red [Mijum16]. La Figura 3.3 muestra dos VNFs que necesitan ser atravesadas en un determinado orden, mientras que en rojo se representa el camino marcado en la red de trasporte que pasa por tales VNFs en el orden determinado, entre los nodos origen y destino, de esta forma satisfaciendo la demanda del servicio de red.

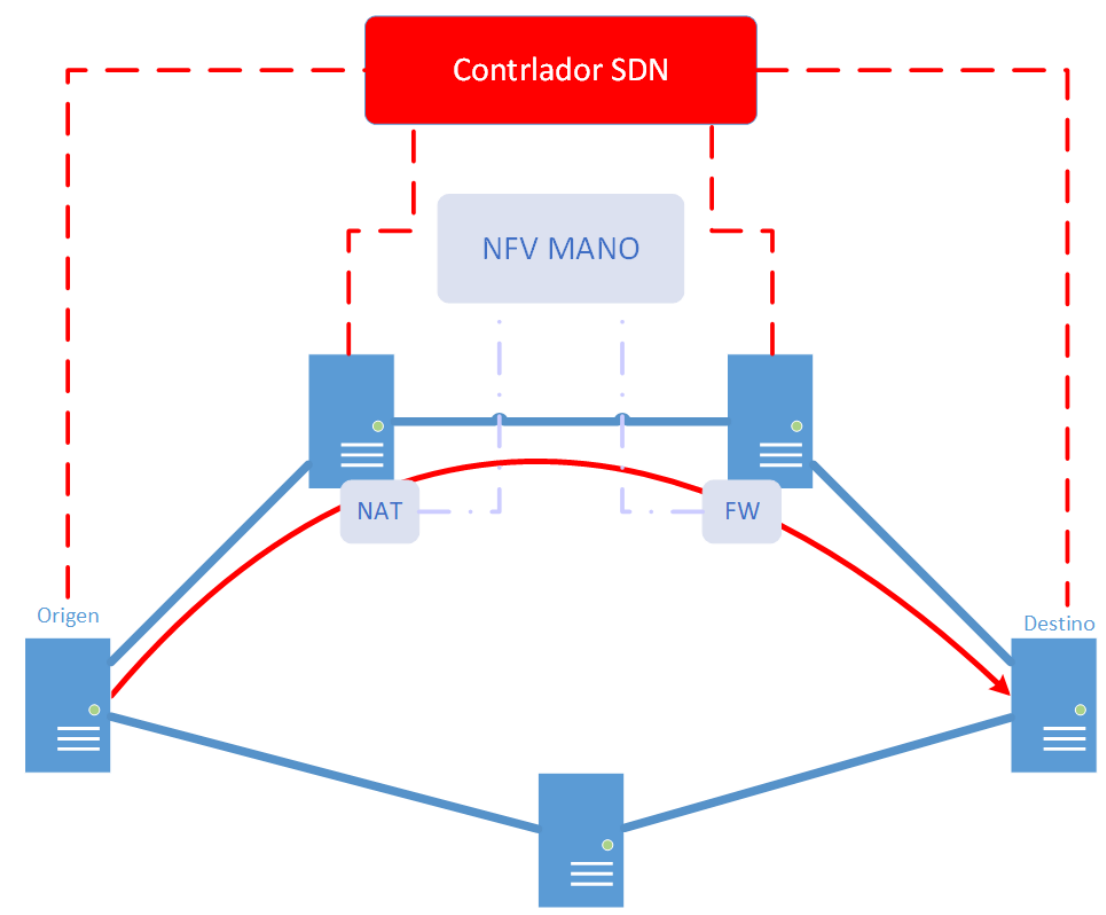

Figura 3.3: Ejemplo de service chain en un entorno de combinación SDN-NFV.

En el caso que se expone, el NFV MANO se encarga de la instanciación de las VNFs 
en las localizaciones (Virtual Infrastructure Managers, VIMs) deseadas dentro del NFVI, teniendo en cuenta el orden de la cadena. Por otra parte, el controlador SDN configura correctamente aquellos dispositivos en el plano de control que formen parte de la ruta que satisfaga tal servicio de red, reservando y asignando los recursos necesarios en la red de transporte desde una perspectiva centralizada.

\subsection{Motivación de uso y herramientas open source}

El paradigma de desarrollo de software dentro del marco del control y la gestión de redes está cambiando considerablemente como consecuencia del aumento del uso de herramientas basadas en código abierto o open source [Linux]. Frente al uso de herramientas de código propietario, el entorno open source se alza como una solución efectiva para abordar los desafíos actuales y futuros en diversos campos, incluyendo la gestión y control de redes de telecomunicaciones, desde una perspectiva de colaboración abierta sin restricción de participación, favoreciendo la proliferación de multitud de herramientas específicas para las distintas tareas necesarias [OpenN18]. Esta visión de desarrollo, permite una evolución de las herramientas en paralelo, mientras toda la comunidad se ve favorecida de los avances conseguidos de forma casi inmediata gracias a la facilidad de acceso a tales herramientas.

Uno de los objetivos principales de la presente tesis es poner en valor el uso de las herramientas de código abierto en el campo de la gestión y control de redes de telecomunicaciones, poniendo el foco especialmente en el uso e interacción de software dedicado a un entorno SDN-NFV. En esta subsección se enuncian algunas de las principales herramientas relativas a SDN o NFV haciendo especial hincapié en aquellas utilizadas en el transcurso de la presente tesis.

\subsubsection{Controladores SDN}

Como se ha presentado anteriormente, el controlador SDN es la pieza central de todo sistema de gestión de redes controladas por software. Multitud de proyectos open source orientados al desarrollo de controladores SDN coexisten en la actualidad creando un amalgama de posibilidades para la comunidad. En los orígenes de SDN, el protocolo OpenFlow era tan ampliamente utilizado para control de switches y routers que era ciertamente común referirse como controlador SDN o OpenFlow indistintamente [Shali13][Salma16]. Algunos ejemplos de controladores SDN/OpenFlow mas populares eran, NOX [Nox], POX [Pox], Beacon [Beaco] o Floodlight [Flood18].

Bajo la el contexto de la Linux Foundation, se presenta el proyecto Open Daylight (ODL), una plataforma abierta basada en Java organizada mediante módulos enfocada a maximizar la capacidad de programación de la red [OpenD]. Open Daylight se caracteriza por presentar un alto grado de personalización y automatización ademas de ser una de las soluciones SDN mas utilizadas en la actualidad. Cisco Digital Network Architecture (Cisco DNA) es una propuesta de red controlada mediante software orientada a automatizar los servicios de red en redes tipo WAN o en grandes campos empresariales [Cisco16b]. Dentro del marco de Cisco DNA existe un módulo específico que actúa como controlador 
SDN orientado a la automatización de infraestructuras de redes WAN para facilitar el las operaciones de red y el despliegue de aplicaciones [Cisco18]. Otra opción relevante como controlador SDN es ONOS, esta herramienta ha sido la elegida para el desarrollo de esta parte de la tesis.

\subsubsection{Open Networking Operating System (ONOS)}

The Open Networking Operating System (ONOS) es el controlador SDN resultante del proyecto ONOS, liderado por la Open Networking Foundation [Onos]. ONOS se caracteriza por ser una plataforma SDN que incluye un amplio conjunto de aplicaciones, facilitando también el desarrollo por parte de los usuarios. Además, ONOS admite tanto la configuración como el control en tiempo real de la red, eliminando la necesidad de ejecutar protocolos de control de enrutamiento y conmutación dentro del tejido de la red. Es una herramienta donde la colaboración de la comunidad es muy amplia y activa.

Gran cantidad de entidades (Huawei, Telefónica, Samsung, Ciena) y proyectos (ACINO, Metro-Haul) utilizan ONOS para realizar diversas tareas dentro de distintos marcos contextuales como control de redes SDN, control de redes de transporte (IP/óptica) o incluso como parte de productos comerciales finales [Onos]. ONOS ha sido elegido para llevar a cabo la integración de la parte de control SDN en la presente tesis. Esta elección ha sido condicionada por el proyecto europeo H2020 Metro-Haul, uno de los proyectos en los que se ha enmarcado esta tesis.

\subsubsection{Orquestadores NFV}

Dentro de la gestión de recursos NFV en código abierto existen dos grandes proyectos en la actualidad. The Open Network Automation Platform es una plataforma de gestión y control de recursos virtualizados que viene respaldada por la Linux Foundation. ONAP proporciona un marco único para el diseño, implementación, análisis y gestión de ciclo de vida de VNFs a gran escala [Onap]. ONAP sigue la arquitectura propuesta por la ETSI para funcionalidades, interfaces y bloques funcionales. La pertenencia a la Linux Foundation facilita la integración de ONAP con otras herramientas de tal fundación. Tacker es un proyecto enmarcado dentro de OpenStack y orientado a la gestión de NFV [OpenS].

La otra opción relevante actualmente para el papel de orquestrador NFV es Open Source Mano (OSM), liderada por la ETSI. La presente tesis utiliza OSM para su implementación. Mayor nivel de detalle a continuación.

\subsubsection{Open Source MANO (OSM)}

La herramienta de gestión y control de recursos virtualizados OSM deriva de un proyecto anterior llamado Open MANO, liderado por Telefónica. Desde 2016, este proyecto se enmarca dentro del contexto de la ETSI, ya bajo el nombre Open Source MANO [Osm]. Se trata de una iniciativa en código abierto con mas de 100 entidades involucradas en su desarrollo y mas de 50 organizaciones forman parte como miembros del proyecto. En la escritura de esta tesis, la última versión disponible es la 4 (Release FOUR). OSM forma parte importante de la estructura de la propuesta del proyecto Metro Haul, de tal forma 
que, para ir en línea con tal proyecto, se ha elegido ETSI OSM (Release THREE) como NFVO para el desarrollo de este trabajo.

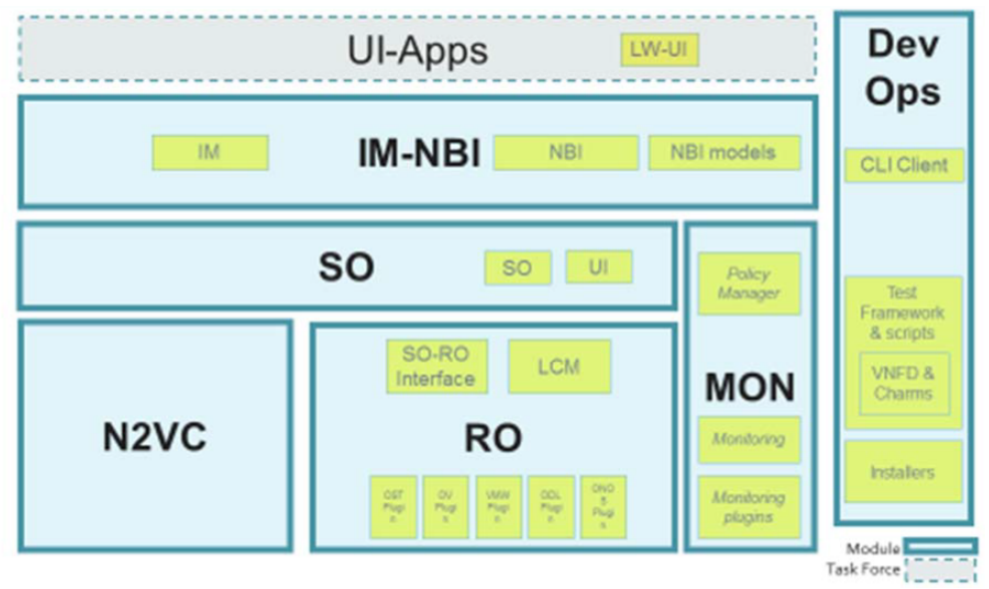

Figura 3.4: Estructura de bloques de OSM Release FOUR. Fuente [Osm].

La Figura 3.4 muestra el esquema funcional de OSM en la versión cuarta. Las funcionalidades básicas de este NFVO se engloban en tres grandes bloques:

- RO (Resource Orchestrator) es el encargado de la gestión y coordinación de la asignación de recursos en el NFVI y la interacción con los VIMs y los controladores SDN.

- N2VC (Network to VNF Configuration) es responsable de la gestión y configuración de las VNFs. Es una evolución del bloque funcional llamado VCA (VNF Configuration and Abstraction) en la versión 3.

- SO (Service Orchestrator) habilita y simplifica las fases involucradas en un servicio complejo NFV desde una perspectiva superior al RO y N2VC.

El uso de OSM se puede realizar desde la capa de aplicaciones gracias a la existencia de una API que permite acceder a las funcionalidades aportadas por este NFVO. Tiene compatibilidad con múltiples tipos de VIMs como, OpenVIM, Amazon Web Services, VMWare u OpenStack.

\subsubsection{Gestores de infraestructuras virtuales, VIMs}

La gestión de los recursos dados por las infraestructuras orientadas a la virtualización está experimentando un gran auge en los últimos años, especialmente en lo relativo a código abierto. Los VIMs se encargan de configurar los recursos de computación y virtualización a través de multitud redes con presencia de datacenters [Mijum16]. Herramientas de software como OpenVIM, VMWare u OpenStack están ayudando a soportar a nivel estructural los avances en el entorno de la virtualización de redes. Este último ha sido el utilizado para hacer el papel de VIM en este trabajo. 


\subsubsection{OpenStack}

El programa de gestión de recursos virtuales, OpenStack, puede controlar una gran cantidad de recursos de computación, almacenamiento, memoria e incluso redes dentro de un datacenter [OpenS]. Es una de las opciones de código abierto mas consideradas tanto para empresas como para la academia. Su estructura modular hace a OpenStack una herramienta muy versátil dónde los módulos agrupan gran cantidad de funcionalidades para poder ser utilizados de forma individual por agentes en niveles superiores gracias a su API. Los principales proyectos/módulos que componen OpenStack son los siguientes:

- Nova es el proyecto que se encarga de proporcionar acceso al servicio automático a los recursos de cómputo, incluidos tanto recursos virtuales como físicos.

- Neutron ofrece la gestión de redes como servicio (Networking-as-a-service, NaaS) dentro de un entorno virtualizado.

- Swift está diseñado para almacenar datos de forma eficiente, segura y barata.

- Glance es el encargado de gestionar las imágenes de las máquinas virtuales.

- Keystone aporta un servicio de autentificación y autorización para múltiples usuarios o contextos.

- Cinder es el servicio de almacenamiento por bloques que se proporciona a los usuarios sin necesidad de conocer la localización de dicho almacenamiento.

Dada su gran facilidad de uso y su conectividad gracias a la API, OpenStack ha sido elegido para realizar las labores de VIM en el marco de la presente tesis.

\subsection{Optimización en entornos SDN-NFV}

En esta sección se presentan las principales aportaciones derivadas de [Garri18a]. En tal contribución académica se realiza un esfuerzo de investigación del estado del arte dónde se exponen y se discuten diversas iniciativas relevantes dentro del ecosistema SDNNFV, donde la optimización puede jugar un papel determinante, presentándose un caso de uso ilustrativo para cada iniciativa. Esta sección se divide en cuatro casos de uso que ilustran el potencial de los sistemas basados en SDN-NFV: a) control de la capa óptica de transporte, b) control de redes de trasporte IP sobre WDM, c) asignación de recursos de computación (IT) y d) asignación de recursos de red y computación conjuntamente en redes de transporte.

\subsubsection{Optimización en capa óptica de transporte}

Tradicionalmente, los grandes operadores de redes de telecomunicaciones se han caracterizado por tener una integración vertical, esto significa que los fabricantes de equipamiento de red aportan conjuntamente sus propias funcionalidades en la capa de control, normalmente con software propietario aportado por el propio fabricante. Este enfoque 
crea cierta dependencia en el operador de la que es difícil desprenderse aportando una carencia de flexibilidad y personalización en el sistema. Este hecho es comúnmente conocido como vendor islands. Con el fin de evitar en la medida de lo posible esta dependencia, se hace necesaria la creación de sistemas abiertos en las redes de transporte a nivel óptico para favorecer la optimización y flexibilidad de tal entorno.

Bajo este contexto, se están alzando multitud de iniciativas. Una de las ramas de investigación en este marco se enfoca en la apertura de las APIs en la interfaz sur en una red óptica SDN que aporte independencia de los fabricantes [Ricca18]. Por una parte el protocolo NETCONF establece las principales operaciones en configuración y monitorización de estado de los dispositivos en el plano de datos, además de la posibilidad de expansión de funcionalidades, dada su naturaleza abierta [Enns11]. El otro pilar principal en la apertura de APIs SBI, es el modelado de los dispositivos ópticos, el modelo YANG se presenta como una alternativa confiable y abierta para describir los dispositivos independientemente de su fabricante. Teniendo ambos principios en cuenta, se pueden desarrollar APIs abiertas basadas en el protocolo NETCONF que procesen las descripciones de los dispositivos ópticos modeladas en YANG [Bjork10].

Existen multitud de proyectos que abordan este enfoque conjunto NETCONF-YANG. El proyecto OpenConfig proporciona un modelo de datos común para la gestión de interfaces tal que los operadores puedan configurar y monitorizar utilizando equipamiento, no solo óptico, sin importar el fabricante [OpenC]. Por otro lado, OpenROADM [OpenR] define ciertas reglas de interoperabilidad óptica aportando modelos YANG con el objetivo de ser equipamiento intercambiable y funcional respecto a APIs basadas en NETCONFYANG y controladas mediante software. Finalmente, el proyecto OpenDevice adapta ciertas propuestas dadas por OpenROADM y OpenConfig para expandir las funcionalidades dadas [Yilma18].

\subsubsection{Caso de uso: optimización de QoT}

En la Figura 3.5 se muestra una representación esquemática de una posible implementación para aportar optimización en la capa óptica. El presente caso de uso contempla como entrada un conjunto de parámetros propios de dispositivos ópticos modelados en YANG que aporten información sobre métricas como potencia de transmisión, OSNR, ganancia de los amplificadores, etc. y una comunicación con el plano de control (controlador SDN) gracias a NETCONF a través de la SBI. Debido a la información de los dispositivos aportados por el controlador SDN, se puede ejecutar un algoritmo que optimice la calidad de transmisión (Quality of Transmission, QoT) y devuelva la salida (por ejemplo, la ruta en la red óptica o la potencia de los traspondedores o los amplificadores) al controlador SDN, que a su vez, configure los dispositivos ópticos de tal manera que la transmisión del lightpath correspondiente esté optimizada según el resultado proveniente de la salida del algoritmo.

\subsubsection{Optimización de redes de transporte IP sobre WDM}

Las redes de transporte IP sobre WDM (Wavelength Division Multiplexing) en un sistema controlado por software es totalmente susceptible de optimización o planificación 


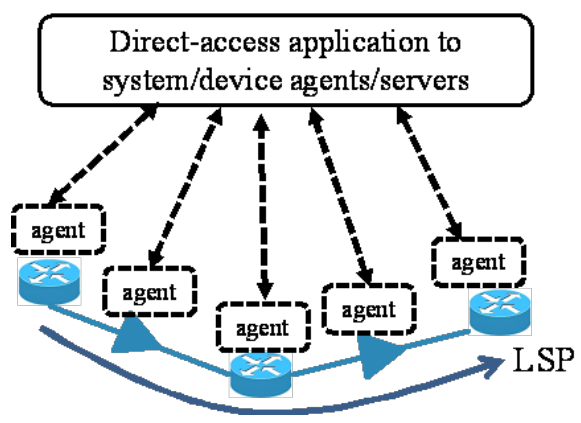

Figura 3.5: Caso de uso: optimización de QoT en redes SDN. Fuente: [Garri18a].

a largo plazo [Gerst15]. La separación entre los planos de control y datos proporcionado por el entorno SDN habilita el uso de aplicaciones en un nivel superior que pueda proporcionar eficiencia al sistema, como por ejemplo, aplicaciones basadas en la optimización como servicio (Optimization as a Service, OaaS). Estas aplicaciones utilizan la interfaz norte para comunicarse con el controlador SDN y enviar los resultados de una hipotética decisión sobre como asignar los recursos en el plano de datos.

\subsubsection{Caso de uso: optimización de recursos en redes SDN.}

La Figura 3.6 expone un caso de uso donde se trata de dar solución al problema de la asignación de recursos y planificación de la red a largo plazo. Desde la capa de aplicación, se pueden aportar soluciones a dichas tareas aportando optimización como servicio (OaaS), necesitando como entradas los parámetros necesarios dados por el controlador SDN según la NBI (interfaz norte). La aplicación traslada sus resultados de vuelta a través de la NBI al controlador SDN que se encarga de configurar correctamente los dispositivos tanto en capa IP como óptica.

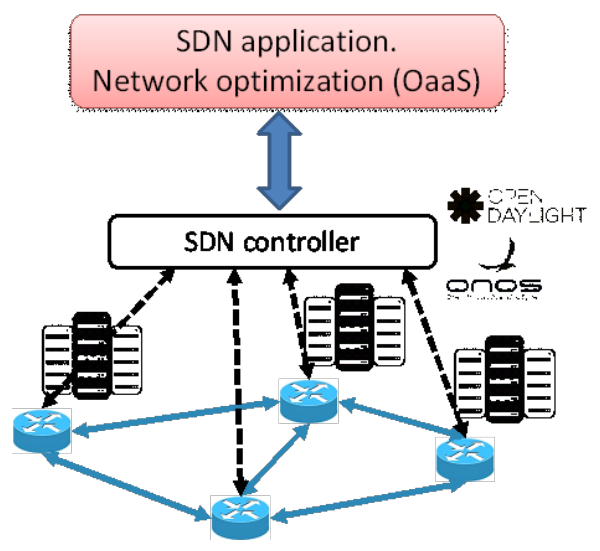

Figura 3.6: Caso de uso: optimización de recursos en redes SDN. Fuente: [Garri18a]. 


\subsubsection{Optimización en la asignación de recursos IT}

El paradigma actual de la computación en la nube ofrece parte de la infraestructura como servicio, IaaS (Infraestructure as a Service), virtualizando los recursos físicos tradicionales en los centros de datos para ofrecerlos como servicio a los usuarios. Tal funcionalidad se basa en la instanciación, gestión de vida e interconexión de las máquinas virtuales que consumen los recursos virtuales del datacenter.

Como se explica en secciones anteriores, este conjunto de tareas está a cargo del VIM dentro del marco NFV. OpenStack se presenta como la opción open source mas popular. Dentro de OpenStack, el proyecto Nova es el encargado de la computación y proporciona una API abierta con la que agentes externos pueden acceder a tal servicio [OpenS]. Por otra parte, Heat es el servicio de OpenStack que ofrece soluciones para asignar recursos de computación o de red conjuntamente a diversas VMs (o VNFs). Finalmente, el proyecto Watcher de OpenStack tiene como objetivo optimizar los recursos de manera flexible y escalable incluyendo un repositorio de métricas y sistema de optimización para procesamiento viéndose beneficiado de la información monitorizada por el servicio Ceilometer. Net2plan-OpenStack es un módulo que está siendo desarrollado por el grupo GIRTEL de la UPCT para optimizar VMs y gestionar recursos dentro de OpenStack [Garri19b].

\subsubsection{Caso de uso: optimización de recursos para VNFs}

La optimización en la asignación de recursos para una VM (o VNF) dentro de un único VIM se muestra en la Figura 3.7a). En este caso de uso, las entradas en un hipotético algoritmo de optimización serían: (a) los requisitos de la máquina virtual en términos de CPU, HD, RAM y (b) el conjunto de datos derivados de la ejecución de la VNF en tiempo real a nivel de arquitectura interna, conocido como EPA (Enhanced Platform Awareness), alguno de estos parámetros pueden ser el tipo de arquitectura de la CPU, tasa de datos de los buses utilizados (físicos y virtuales), etc. Este último conjunto de información es especialmente relevante para la instanciación de VNFs con baja latencia, por lo que optimizar la asignación de recursos virtuales en este entorno se asume como esencial. Por otra parte, la imagen 3.7b) ilustra la optimización de una cadena de servicios (con varias VNFs) en el dominio de un único VIM. En este caso, para la salida, además de la asignación de recursos virtuales, es necesario resolver el problema de las interconexiones entre los propios VNFs para poder crear la service chain.

\subsubsection{Optimización conjunta para recursos de red e IT en redes de transporte}

En la actualidad, las redes de transporte están evolucionando rápidamente hacia un sistema distribuido de datacenters, conjuntamente diseñado para explotar los beneficios SDN-NFV. Como se expuso en el capítulo 2, alojar contenido cerca de los usuarios finales puede aliviar parte del tráfico atravesado en las redes tipo backbone o core [Pavon17] [Moren18a]. Este ecosistema se puede ver claramente beneficiado por la implementación óptima de redes basadas en SDN y NFV.

Iniciativas open source como CORD (Central Office Re-archiectured as Datacenter) [Cord], proyecto perteneciente a la Open Networking Foundation, están recibiendo el 


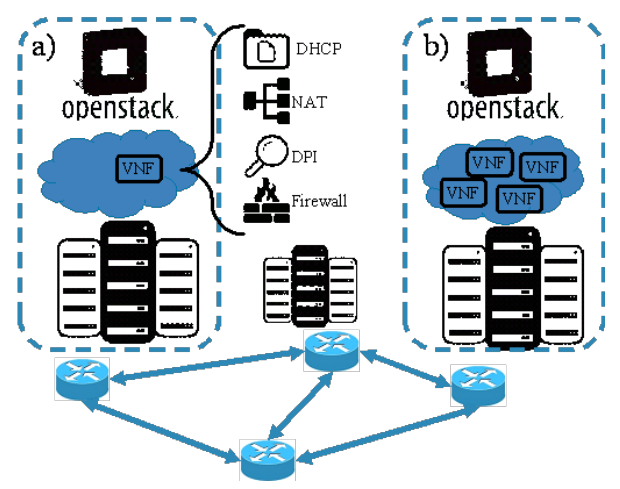

Figura 3.7: Caso de uso: optimización de recursos IT para, a) una VNF, b) varias VNFs. Fuente: [Garri18a].

interés de los operadores de telecomunicaciones bajo la promesa de obtener beneficios en agilidad, versatilidad, eficiencia o rendimiento. El proyecto CORD está íntimamente relacionado con el controlador SDN, ONOS.

En un ambiente de SDN/NFV, existen dos grandes proyectos que tienen bajo consideración los recursos IT propios de los datacenters conectados a redes de transporte: ETSI OSM y ONAP, ambos expuestos en secciones anteriores. Está previsto que OSM permita la instanciación de múltiples VNFs en varios datacenters creando la conectividad entre ellos de forma automática. Esta nueva funcionalidad será encargada al llamado WIM (WAN Infrastructure Manager), está en actual desarrollo y está previsto su lanzamiento para la quinta versión de OSM. El WIM es producto del desarrollo del proyecto Metro-Haul en colaboración con la comunidad de OSM. Dentro del marco Metro-Haul, la optimización y asignación conjunta de recursos IT y de red para las VNFs está llevado a cabo por Net2plan, proporcionando OaaS.

\subsubsection{Caso de uso: optimización de service chain con múltiples VIMs conectados a una red de trasporte}

En la Figura 3.8 se puede visualizar un caso de uso en el que es necesario gestionar de forma eficiente la asignación y localización (distintos VIMs) de las VNFs pertenecientes una service chain dentro de un contexto de virtualización sobre una red de transporte. En un sistema SDN-NFV dado por un controlador SDN en comunicación con un NVF MANO, que gestiona la parte de virtualización, una aplicación que reciba el panorama completo del sistema puede instruir tanto al controlador SDN para elegir la ruta óptima en la red de transporte, como al NFV MANO a la hora de seleccionar los VIMs correctos para las VNFs que optimice el camino completo de la service chain.

Éste último caso de uso para la optimización de un sistema SDN-NFV, ha servido como base para la creación de dos pruebas de concepto presentadas como demostraciones en dos congresos internacionales [Moren18b] y [Moren18d]. Tales pruebas de concepto se exponen detalladamente en la siguiente sección. 


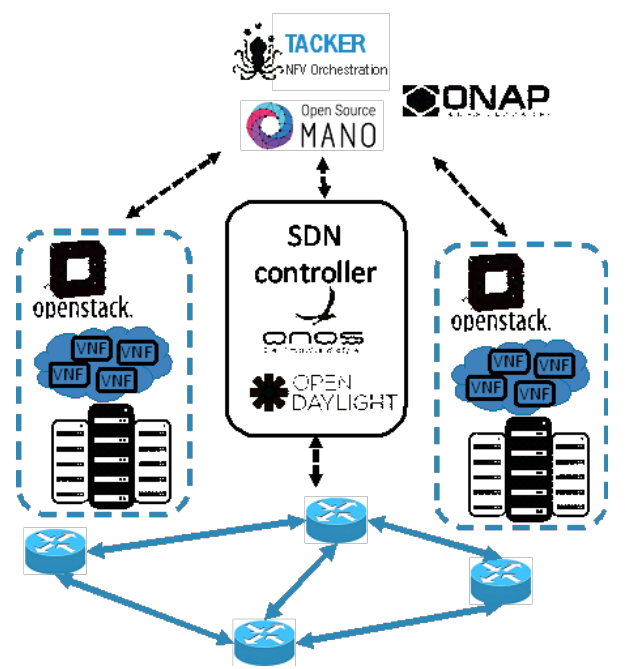

Figura 3.8: Caso de uso: optimización de service chain con múltiples VIMs conectados a una red de trasporte. Fuente: [Garri18a].

\subsection{Gestión en SDN-NFV. Pruebas de concepto}

Esta sección enuncia y explica en detalle las dos pruebas de concepto realizadas bajo el contexto de la presente tesis doctoral orientadas la gestión y optimización de sistemas basados en SDN y NFV, utilizando para su desarrollo únicamente herramientas en código abierto. Los resultados de ambas pruebas de concepto han sido presentadas como demostraciones en [Moren18b] y [Moren18d].

\subsubsection{Demostración de asignación conjunta de cadena de servicios, instanciación de VNFs y gestión de recursos en redes metro}

Esta demostración es una prueba de concepto en un entorno dinámico NFV sobre el que la herramienta de planificación en código abierto Net2plan se encarga de instruir a el orquestrador NFV, representado por ETSI OSM (Release THREE), en la tarea de instanciación de VNFs componentes a cadenas de servicios (service chains), desde una perspectiva eficiente, en tres VIMs (OpenStack) conectados a una red de transporte básica emulada mediante Net2plan.

\subsubsection{Esquema funcional de la demostración}

La Figura 3.9 muestra el esquema de bloques propuesto para llevar a cabo la empresa de esta demostración. A continuación se exponen los principales componentes funcionales utilizados:

- Operation Support System (OSS): este bloque representa un operador de red que necesita dar servicio al tráfico de aplicaciones dentro del marco NFV. Es quien representa el papel de usuario en esta demostración, definiendo las demandas a satisfacer. Tal elemento está simulado mediante una interfaz gráfica de usuario (GUI) como plug-in de la herramienta de planificación Net2plan. 
- Orquestrador NFV (NFVO): es la parte central del sistema de virtualización donde se listan y gestionan las VNFs disponibles para ser requeridas por las demandas de usuario e instanciadas en los VIMs. La herramienta open source OSM es la elegida para tal propósito.

- Red de transporte: Se considera una red emulada a nivel IP con funcionalidad básica representada por un grafo de nodos y enlaces, con recursos de red a consumir, como capacidad de los enlaces. Se asume que algunos de los nodos están conectados a VIMs. La topología elegida ha sido NSFNet y está disponible en Net2plan.

- Gestor de Infraestructura Virtual (VIM): existen tres VIMs para alojar y gestionar correctamente las máquinas virtuales asociadas las VNFs a instanciar. Este papel lo realiza el software OpenStack.

- Elemento de computación de cadenas de servicio: o SCCE (Service Chain Computation Element), es el elemento funcional que presenta la inteligencia necesaria para realizar la correcta asignación de las VNFs que componen una cadena de servicios, mientras propone un camino en la red de transporte que satisfaga la demanda atravesando las localizaciones de las VNFs en el orden apropiado. Se trata de una evolución orientada al entorno NFV del elemento bien conocido PCE (Path Computation Element). El comportamiento del SCCE viene modelado como un algoritmo de optimización programado en Net2plan.

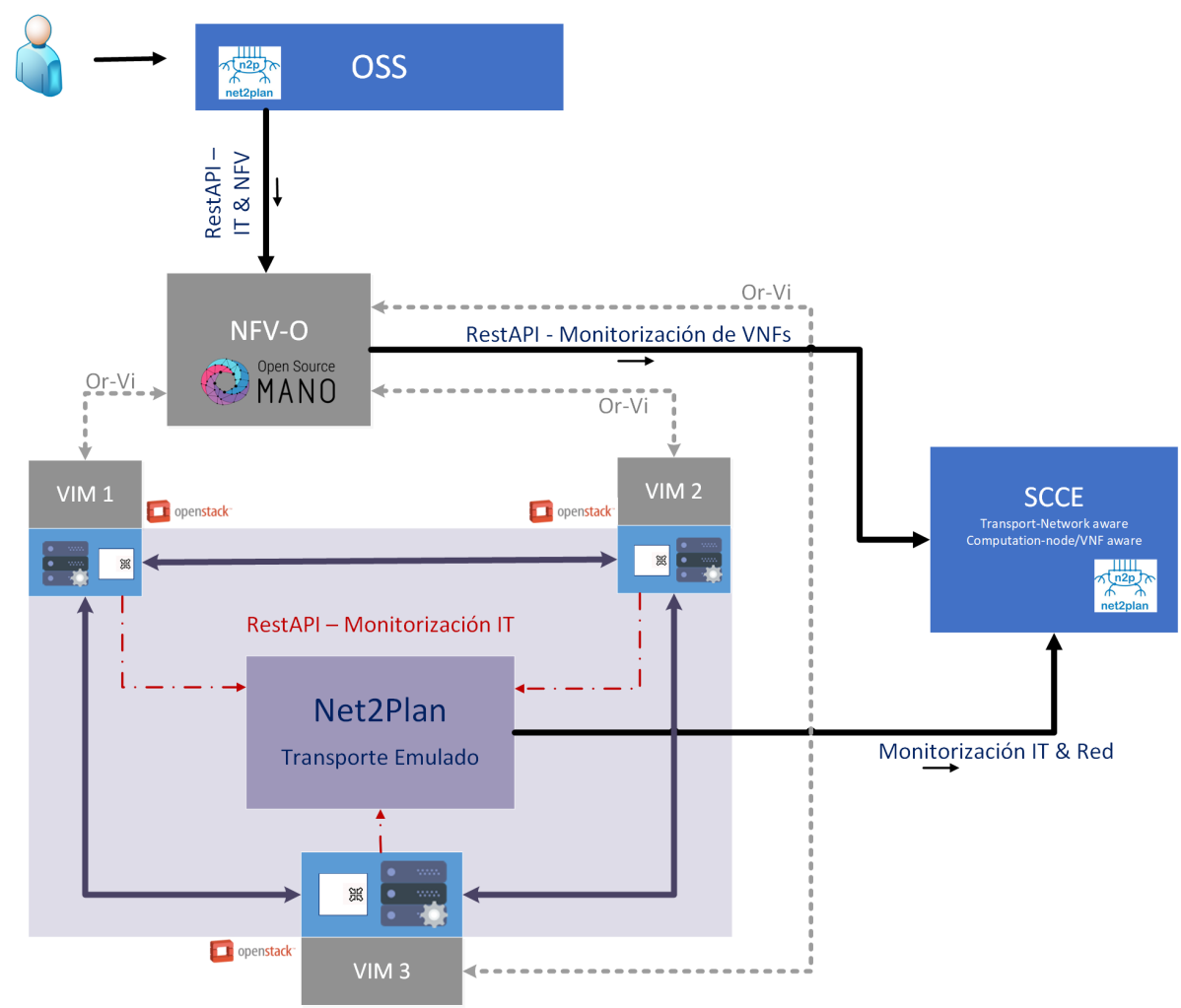

Figura 3.9: Esquema funcional de la prueba de concepto presentada en [Moren18b]. 


\subsubsection{Implementación y configuración}

Para realizar la propuesta de demostración definida anteriormente, se ha implementado el sistema según menciona la Figura 3.10. En primer lugar se ha necesitado de un portátil personal (Intel i7 4720HQ, 16 GB RAM, 240 SSD + 2TB HDD) y tres miniPCs de alto rendimiento (Intel i5 7500, 4 CPUs, 8 GB RAM y 240 SSD), dónde Net2plan ha sido instalado en el portátil, una imagen de OSM (Release THREE) instalada en una máquina virtual dentro del propio portátil, mientras que en cada uno de los miniPCs se ha instalado una distribución DevStack de OpenStack (versión Pike) para realizar las tareas de VIMs.

Por otra parte, se ha definido una red (10.0.2.0/24) para interconectar todo el equipamiento bajo la misma gracias a un switch Ehternet autoconfigurable. Esta red representa la conectividad del plano de control en un modelo SDN-NFV. Desde el punto de vista del networking virtual, como se muestra en la Figura 3.10, se ha definido una estructura de redes interna en cada OpenStack con una red privada (192.168.1.0/24) donde alojar las máquinas virtuales de las VNFs que a su vez está conectada a través de un router virtual a una red pública que ofrece un conjunto de IPs flotantes que se pueden asignar a las VMs asociadas a las VNFs, obteniendo de esta manera conectividad fuera del VIM.

La definición de las interfaces de comunicación entre Net2plan y los distintos agentes involucrados ha sido parte fundamental del desarrollo de este trabajo. Estas tareas fueron realizadas por el grupo de investigación GIRTEL de la UPCT. La interfaz de Net2plan con OSM se ha basado en el código del cliente de la versión 3 de OSM, pero traducido desde el lenguaje de programación Python a Java. Por otra parte, la comunicación con OpenStack se ha implementado un cliente OpenStack-Net2plan gracias al uso de la librería de código abierto OpenStack4J, que facilita el uso de la API de OpenStack en lenguaje Java.

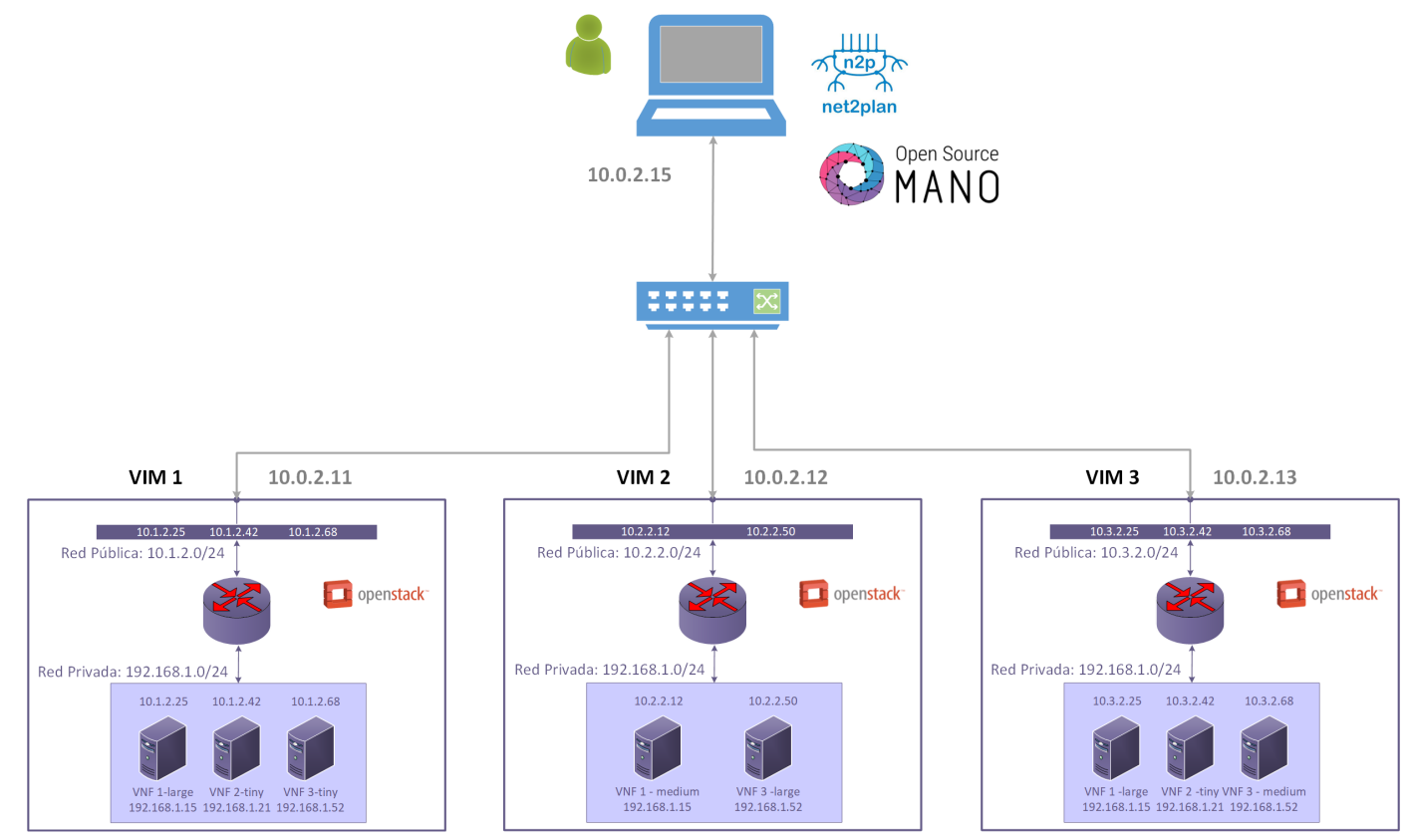

Figura 3.10: Esquema de la implementación de la demostración [Moren18b].

Respecto a la parte de la configuración de las VNFs, se han creado distintos descriptores en OSM con ciertas funcionalidades automatizadas como la asignación de IP flotante 
Tabla 3.1: Lista de VNFs en [Moren18b].

\begin{tabular}{|c|c|c|c|}
\hline Nombre VNF & CPU & RAM & HDD \\
\hline vnf1-ns-tiny & 1 & 0.5 & 5 \\
\hline vnf1-ns-medium & 1 & 1 & 10 \\
\hline vnf1-ns-large & 2 & 2 & 20 \\
\hline vnf2-ns-tiny & 1 & 0.5 & 5 \\
\hline vnf2-ns-medium & 1 & 1 & 10 \\
\hline vnf2-ns-large & 2 & 2 & 20 \\
\hline vnf3-ns-tiny & 1 & 0.5 & 5 \\
\hline vnf3-ns-medium & 1 & 1 & 10 \\
\hline vnf3-ns-large & 2 & 2 & 20 \\
\hline
\end{tabular}

o la creación interna de ficheros. Las máquinas virtuales asociadas de las VNFs son imágenes livianas de Ubuntu 16.04 con funcionalidad básica de cloud dada por la librería Cloud-Init. Mediante un script de configuración de Cloud-Init se instruye a OSM para determinar como ha de ser la funcionalidad de la VNF en tiempo de instanciación. Puesto que el análisis de la funcionalidad de las VNF no es objeto de demostración en este trabajo, las VNFs únicamente crean en tiempo de instanciación un fichero donde se resumen ciertas características de la service chain a la que pertenece. Se asumen tres tipos diferentes de VNFs (misma funcionalidad) con tres requisitos de computación necesarios, lo que hace un total de nueve configuraciones distintas para las VNFs como se muestra en la Tabla 3.1.

\subsubsection{Flujo de ejecución y resultados}

El conjunto de pasos seguidos para la ejecución de esta demostración se expone como sigue:

1. En el proceso inicial de carga, Net2plan recibe la información completa de la parte de NFV proporcionada por OSM y de los recursos IT disponibles en cada uno de los VIMs (OpenStack). Conocer el panorama completo del sistema es esencial para realizar la optimización en la asignación de la posterior service chain.

2. Tras realizar correctamente el proceso de carga del sistema y obtención de información, el usuario (OSS), a través de la GUI de Net2plan, define la solicitud de establecimiento de service chain. Para ello, el usuario define arbitrariamente los nodos origen y destino, así como las VNFs ordenadas y un ancho de banda para consumir recursos en los enlaces atravesados.

3. La solicitud de service chain por parte del usuario es recibida como entrada del algoritmo, el cual devuelve como salida la localización de las VNFs en los VIMs y el camino a seguir en la red de transporte. El criterio utilizado por el algoritmo para optimizar la asignación de recursos ha sido calcular el camino mas corto entre los nodos origen y destino pasando por aquellos VIMs que alojen las VNFs en el 
orden correcto, las VNFs se emplazan en los VIMs intentando minimizar el balance de carga computacional entre los VIMs pero cumpliendo siempre el shortest path. Mayor nivel de detalle sobre este algoritmo se expone en la Figura 3.11.

4. Las ubicaciones de las VNFs son notificadas a OSM que instancia las imágenes de tales VNFs en los VIMs (OpenStack) propuestos por el algoritmo.

5. Cuando las máquinas virtuales de las VNFs están activas y con una IP flotante asignadas para poder ser conectadas, se establece una conectividad segura tipo SSH a nivel IP entre las VNFs en el orden establecido por el usuario. Como se aprecia en la Figura 3.12 La GUI de Net2plan se actualiza mostrando el camino elegido para satisfacer la demanda exigida por el usuario.

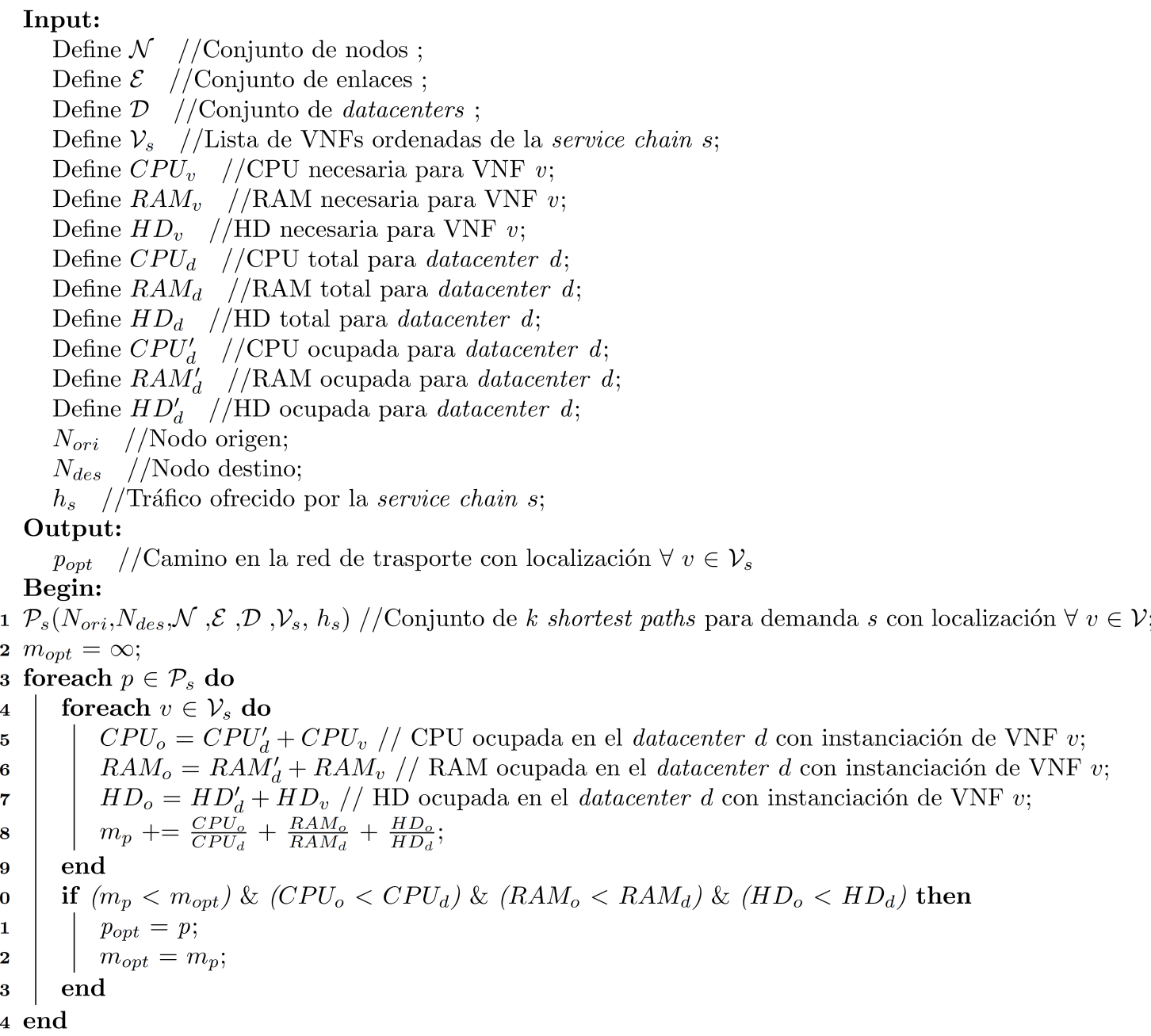

Figura 3.11: Algoritmo para asignación de recursos de red e IT y localización de VNFs en [Moren18b].

Como se ha podido comprobar en este apartado, la expuesta prueba de concepto muestra que es posible realizar tareas de optimización en un entorno NFV con herramientas únicamente de código abierto. La asignación adecuada de recursos virtualizados será clave para poder abordar con garantías la futura era $5 \mathrm{G}$. 


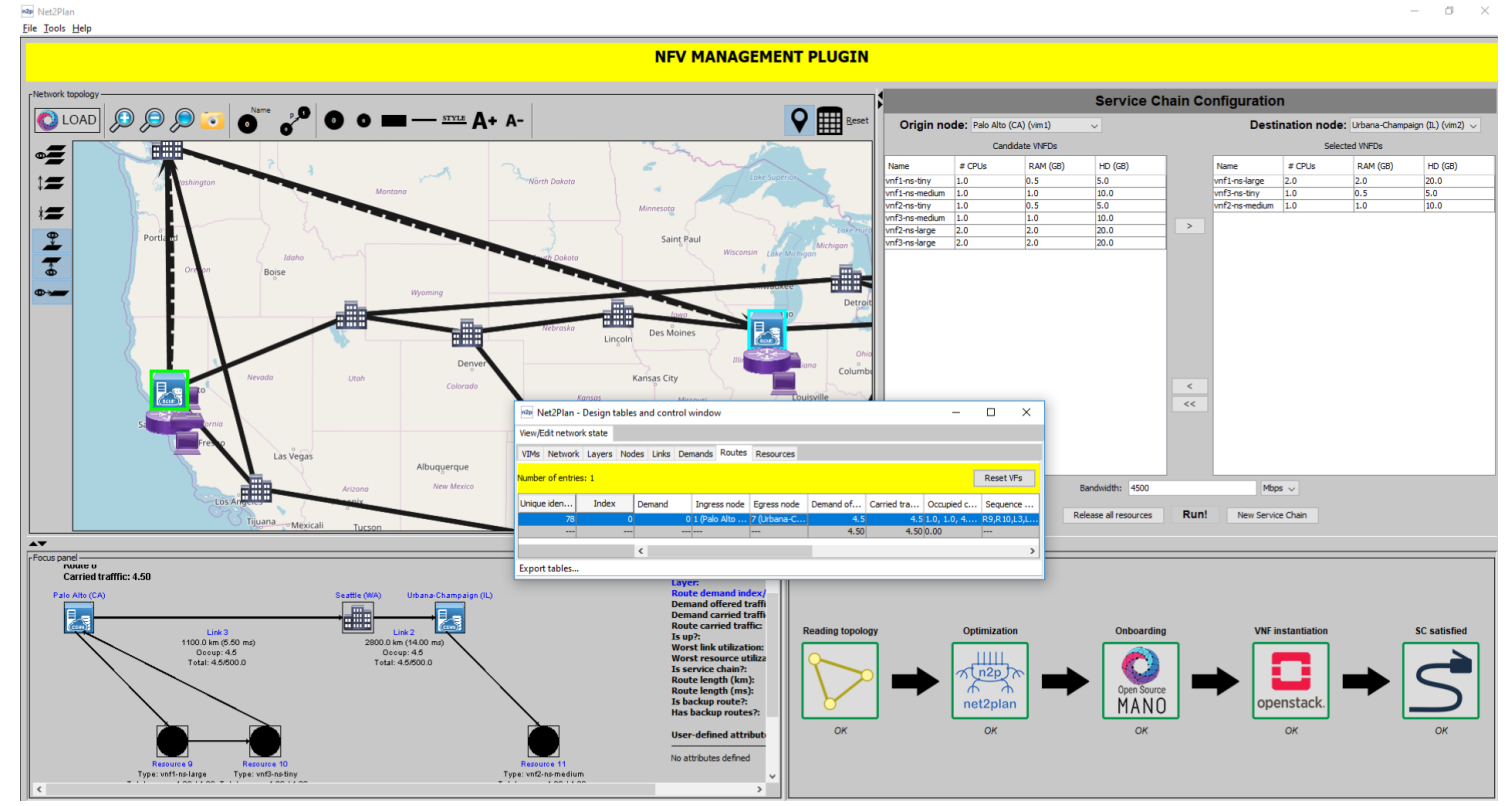

Figura 3.12: Captura en la GUI de Net2plan tras ejecución de la demostración [Moren18b].

\subsubsection{Optimización en service chain basada en latencia con creación de instancias VNFs y control SDN de red metro}

Este trabajo [Moren18d] se puede considerar como una extensión de la demostración presentada en [Moren18b], por lo que gran parte del diseño e implementación deriva del anterior trabajo. Dentro de un contexto SDN-NFV, esta demostración se diferencia en esencia de su precursora en que para este caso se considera que, conjuntamente con el plano de control, el plano de datos entra dentro del marco de estudio, mientras en la prueba de concepto anterior únicamente se centra en detalle en la parte NFV. Además, en [Moren18d] se presenta una modificación del algoritmo para tener en cuenta consideraciones de latencia en las demandas de establecimiento de servicios de red. Este tema se presenta como esencial en el contexto $5 \mathrm{G}$.

\subsubsection{Visión esquemática de la demostración}

La demostración [Moren18d] presenta una prueba de concepto en un entorno dinámico SDN-NFV, donde la herramienta de planificación Net2plan instruye de forma eficiente al NFVO en tareas de instanciación de VNFs y gestión de recursos virtualizados, así como adiestrar a un controlador SDN para que configure de forma adecuada el equipamiento emulado a nivel IP en el plano de datos, tal que permita satisfacer la demanda de los usuarios teniendo en cuenta exigencias de latencia. 3.13 .

El esquema funcional del presente trabajo de investigación se expone en la Figura

Los agentes involucrados en el diseño de esta demostración son los siguientes:

- Operation Support System (OSS): representa un operador de red para proveer el 


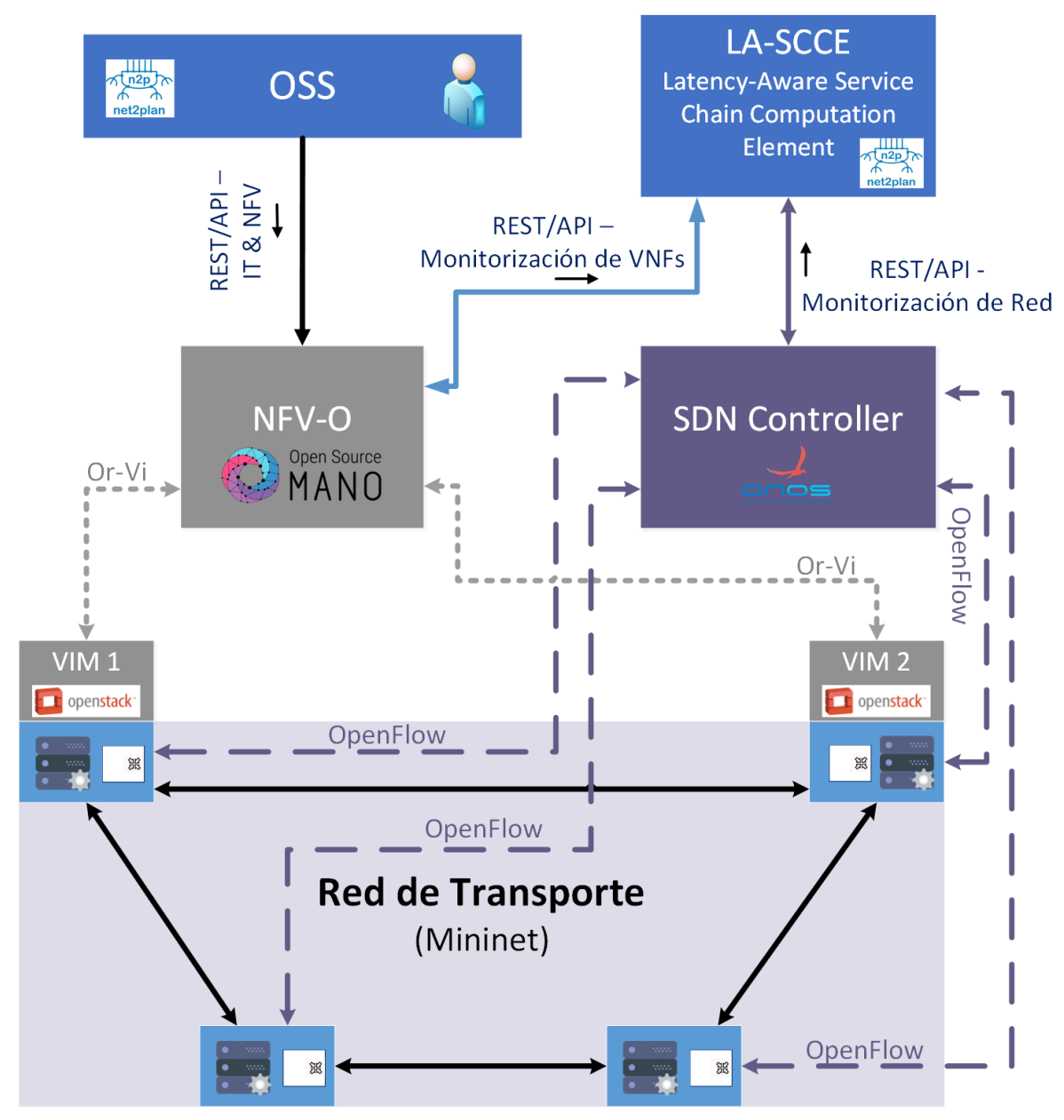

Figura 3.13: Esquema funcional de la demostración [Moren18d].

tráfico de aplicaciones enmarcado en un contexto SDN-NFV. De forma análoga al trabajo previos, representa el papel de usuario en esta demostración. Tal elemento está simulado mediante una interfaz gráfica de usuario (GUI) como plug-in de la herramienta de planificación Net2plan.

- Orquestador NVF (NVFO): representada por OSM quien está a cargo de la infraestructura de virtualización y almacenamiento de los descriptores de las VNFs.

- Red de transporte: La red de transporte a nivel IP viene emulada por la herramienta de código abierto, Mininet, que proporciona un conjunto de hosts y switches virtuales que realizan el papel de nodos en la topología considerada.

- Control SDN: La red metropolitana aportada por Mininet es controlada y configurada a través del controlador SDN, ONOS, gracias al protocolo OpenFlow.

- Gestor de Infraestructura Virtual (VIM): se consideran dos VIMs para alojar y gestionar correctamente las máquinas virtuales relativas a las VNFs. OpenStack es el encargado de tal propósito. Los VIMs están conectados al plano de datos. 
- Elemento de computación de cadenas de servicio basado en latencias: o LASCCE (Service Chain Computation Element), es una evolución del anterior SCCE, en este caso, la decisión en la gestión de recursos de red e IT viene, además, influenciada por las exigencias de latencia especificadas por el OSS. Este algoritmo está implementado en Net2plan.

\subsubsection{Implementación y configuración}

El equipamiento necesario para realizar esta demostración ha sido un portátil personal (Intel i7 4720HQ, 16 GB RAM, 240 GB SSD + 2 TB HDD) y tres miniPCs de alto rendimiento, dos de ellos con Intel i5 7500, 16 GB RAM y 240 GB SSD, mientras que el restante sube hasta 24 GB RAM. El NFVO, OSM (R3), está instanciado en una máquina virtual dentro del portátil, Net2plan también está instalado en dicho ordenador. Mientras que para representar a los dos VIMs, se han utilizados distribuciones PackStack de OpenStack versión Queens en los dos miniPCs con menores recursos, mientras que la red de transporte emulada por Mininet y el controlador SDN, ONOS, están presentes el miniPC de mayor rendimiento.

La Figura 3.14 escenifica la implementación llevada a cabo para la presente prueba de concepto. De forma análoga a la demo anterior, se ha configurado una red de control (10.0.2.0/24) gestionada por un switch autoconfigurable entre los agentes involucrados en el plano de control, representada con la parte azul en el esquema 3.14. Por otra parte, la definición del plano de datos (en verde en Fig. 3.14) se lleva a cabo en dos partes. En primer lugar, la interconexión a través de una red física entre los miniPCs para dar soporte al trasporte de datos (15.0.0.0/16), mientras que se han configurado redes públicas en los VIMs (OpenStack) para que las máquinas virtuales tengan acceso a la red de datos (15.x.0.0./24), gracias a la asignación de IPs flotantes a las VMs, tales VMs se alojan en redes privadas (192.168.1.0/24) en OpenStack.

Este trabajo se ha visto beneficiado por el desarrollo de las interfaces anteriormente expuestas en el anterior trabajo para comunicar Net2plan con OSM y OpenStack, sin embargo, ha sido necesaria la elaboración de un cliente en Java que interactúe con la API de ONOS. La comunicación entre ONOS y Mininet se realiza de forma transparente a Net2plan según la interfaz sur a través de OpenFlow.

Como parte de la infraestructura virtualizada, las VNFs utilizadas son similares a las dadas en [Moren18b], pero en este caso, añadiendo estimaciones arbitrarias, pero realistas, sobre el tiempo de ejecución de las VNFs. Las VNFs utilizadas se muestran en la Tabla 3.2. Se entiende como latencia al tiempo de propagación entre los nodos origen y destino más la suma de los tiempos de ejecución de las VNFs.

\subsubsection{Flujo de ejecución y resultados}

El conjunto de pasos seguidos para la ejecución de la presente demostración se expone como sigue:

1. En el proceso inicial de carga, Net2plan recibe la toda la información tanto de la parte de NFV proporcionada por OSM, como los recursos IT disponibles en cada 


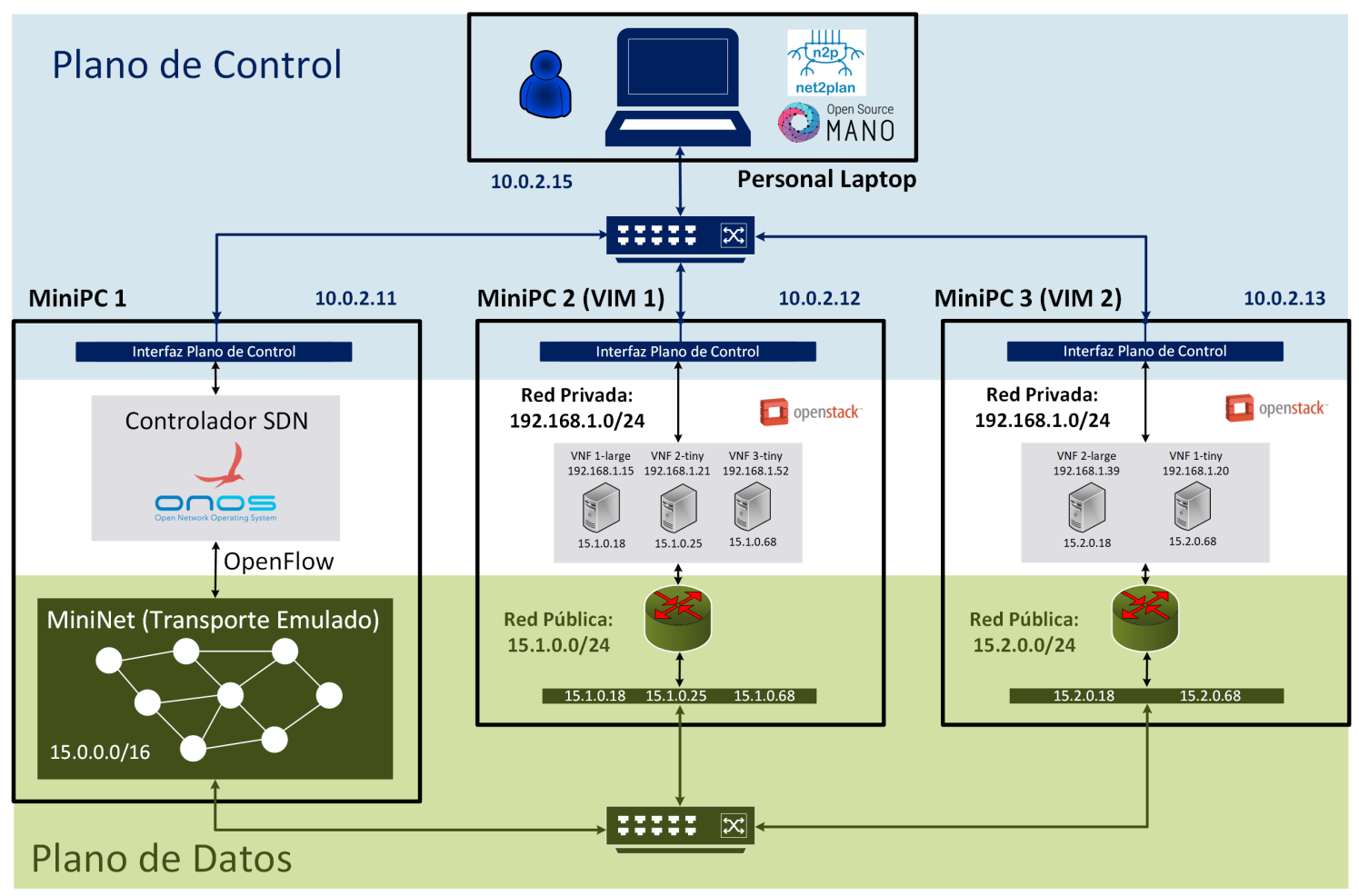

Figura 3.14: Diseño de la implementación de la demostración [Moren18d].

uno de los VIMs (OpenStack), además de la topología de la red de trasporte proporcionada por ONOS, gracias a la RestAPI correspondiente.

2. Tras realizar correctamente el proceso de carga del sistema y obtención de información, el usuario (OSS), a través de la GUI de Net2plan, define la solicitud de establecimiento de service chain. El usuario proporciona arbitrariamente, los nodos origen y destino, así como las VNFs ordenadas, la latencia máxima end-to-end que se debe satisfacer y finalmente, el ancho de banda para consumir recursos en los enlaces de la red de transporte.

3. Los atributos introducidos por el OSS sirven como entradas al algoritmo (ver Figura 3.15 para poder resolver la problemática de dónde alojar las VNFs y qué camino seguir en la red de transporte, y de esta manera poder satisfacer las especificaciones de latencia total entre los nodos origen y destino. En caso de no encontrar ningún camino por dónde satisfacer la demanda, se considera como rechazada. La Figura 3.16 expone el aspecto de la interfaz gráfica de usuario en Net2plan tras haber realizado satisfactoriamente el proceso de algoritmia. Nótese que en los iconos representados en violeta muestran el estado interno (VIM) de la red de OpenStack, ya con las máquinas virtuales pre-instanciadas. 
Tabla 3.2: Lista de VNFs en [Moren18d].

\begin{tabular}{|c|c|c|c|c|}
\hline Nombre VNF & CPU & RAM & HDD & Tiempo ejecución (ms) \\
\hline vnf1-ns-tiny & 1 & 0.5 & 5 & 2 \\
\hline vnf1-ns-medium & 1 & 1 & 10 & 1 \\
\hline vnf1-ns-large & 2 & 2 & 20 & 0.5 \\
\hline vnf2-ns-tiny & 1 & 0.5 & 5 & 2 \\
\hline vnf2-ns-medium & 1 & 1 & 10 & 1 \\
\hline vnf2-ns-large & 2 & 2 & 20 & 0.5 \\
\hline vnf3-ns-tiny & 1 & 0.5 & 5 & 2 \\
\hline vnf3-ns-medium & 1 & 1 & 10 & 1 \\
\hline vnf3-ns-large & 2 & 2 & 20 & 0.5 \\
\hline
\end{tabular}

4. Las ubicaciones de las VNFs son notificadas a OSM que instancia las máquinas virtuales de tales VNFs en los VIMs (OpenStack), según vienen condicionadas por la salida del algoritmo. En la captura 3.17] se puede visualizar el aspecto de la interfaz gráfica de OSM tras realizar exitosamente la instanciación de las dos VNFs propuestas por el usuario en la imagen 3.16

5. Paralelamente al proceso de instanciación de VNFs, Net2plan notifica a ONOS sobre el camino a seguir. En tal situación, ONOS configura, via OpenFlow, los switches virtuales en la red de transporte (Mininet) para que se puedan establecer flujos de tráfico en el plano de datps según el camino propuesto por el algoritmo. En la imagen 3.18 se aprecia en color amarillo los flujos correctamente establecidos entre el nodo origen, en este caso Sevilla, hasta la entrada al datacenter situado en Madrid, según el camino que se visualiza en la captura 3.16 .

6. Tras comprobar el estado activo de las máquinas virtuales de las VNFs, asignadas con una IP flotante, y los dispositivos en Mininet correctamente configurados, se mandan paquetes (comando ping en el sistema operativo) siguiendo la ruta completa entre los nodos origen y destino en Mininet. En la Figura 3.19 se puede comprobar la monitorización de las conexiones establecidas en un determinado switch de Mininet. Los cuatro primeros flujos de la figura se corresponden con comunicaciones controlador-dispositivo mediante el protocolo OpenFlow, mientras que los cuatro restantes hacen referencia al flujo de datos entre los switches virtuales mostrando los puertos de entrada y las direcciones MAC origen y destino para cada flujo de este tipo. Tal procedimiento sirve de validación para comprobar el correcto funcionamiento de la parte de routing en el plano de datos. 


\section{Input:}

Define $\mathcal{N} / /$ Conjunto de nodos ;

Define $\mathcal{E} / /$ Conjunto de enlaces ;

Define $\mathcal{D} / /$ Conjunto de datacenters ;

Define $\mathcal{V}_{s} \quad / /$ Lista de VNFs ordenadas de la service chain $s$;

Define $C P U_{v} \quad / / \mathrm{CPU}$ necesaria para VNF $v$;

Define $R A M_{v} \quad / /$ RAM necesaria para VNF $v$;

Define $H D_{v} \quad / / \mathrm{HD}$ necesaria para VNF $v$;

Define $t_{v} \quad / /$ Tiempo ejecución de VNF $v$;

Define $C P U_{d} \quad / / \mathrm{CPU}$ total para datacenter $d$;

Define $R A M_{d} \quad / /$ RAM total para datacenter $d$;

Define $H D_{d} \quad / /$ HD total para datacenter $d$;

Define $C P U_{d}^{\prime} \quad / /$ CPU ocupada para datacenter $d$;

Define $R A M_{d}^{\prime} \quad / /$ RAM ocupada para datacenter $d$;

Define $H D_{d}^{\prime} \quad / /$ HD ocupada para datacenter $d$;

$N_{\text {ori }}$ //Nodo origen;

$N_{\text {des }} / / /$ Nodo destino;

$h_{s} \quad$ //Tráfico ofrecido por la service chain $s$;

$l_{s} \quad / /$ Latencia máxima aceptada para la service chain $s$;

\section{Output:}

$p_{\text {opt }} / /$ Camino en la red de transporte con localización $\forall v \in \mathcal{V}_{s}$

$$
\text { Begin: }
$$

$1 \mathcal{P}_{s}\left(N_{\text {ori }}, N_{\text {des }}, \mathcal{N}, \mathcal{E}, \mathcal{D}, \mathcal{V}_{s}, h_{s}\right) / /$ Conjunto de $k$ shortest paths para demanda $s$ con localización $\forall v \in \mathcal{V}$;

$2 m_{\text {opt }}=\infty$;

3 foreach $p \in \mathcal{P}_{s}$ do

$4 \quad$ foreach $v \in \mathcal{V}_{s}$ do

5

$C P U_{o}=C P U_{d}^{\prime}+C$

$+C P U_{v} / /$ CPU ocupada en el datacenter $d$ con instanciación de VNF $v$

$R A M_{o}=R A M_{d}^{\prime}+R A M_{v} / /$ RAM ocupada en el datacenter $d$ con instanciación de VNF $v$;

$H D_{o}=H D_{d}^{\prime}+H D_{v} / / \mathrm{HD}$ ocupada en el datacenter $d$ con instanciación de VNF $v$;

$m_{p}+=\frac{C P U_{o}}{C P U_{d}}+\frac{R A M_{o}}{R A M_{d}}+\frac{H D_{o}}{H D_{d}}$

$l_{p}+=\mathrm{t}_{v}$

end

Calcula $t_{p} / /$ Tiempo de propagación del path $p$;

$l_{p}+=t_{p} / /$ Tiempo end-to-end para la service chain por el camino $p$;

if $\left(m_{p}<m_{o p t}\right) \&\left(C P U_{o}<C P U_{d}\right) \&\left(R A M_{o}<R A M_{d}\right) \&\left(H D_{o}<H D_{d}\right) \&\left(l_{p}<l_{s}\right)$ then

$p_{\text {opt }}=p$

$m_{\text {opt }}=m_{p}$

end

7 end

Figura 3.15: Algoritmo para asignación de recursos de red e IT y localización de VNFs en [Moren18d]. 


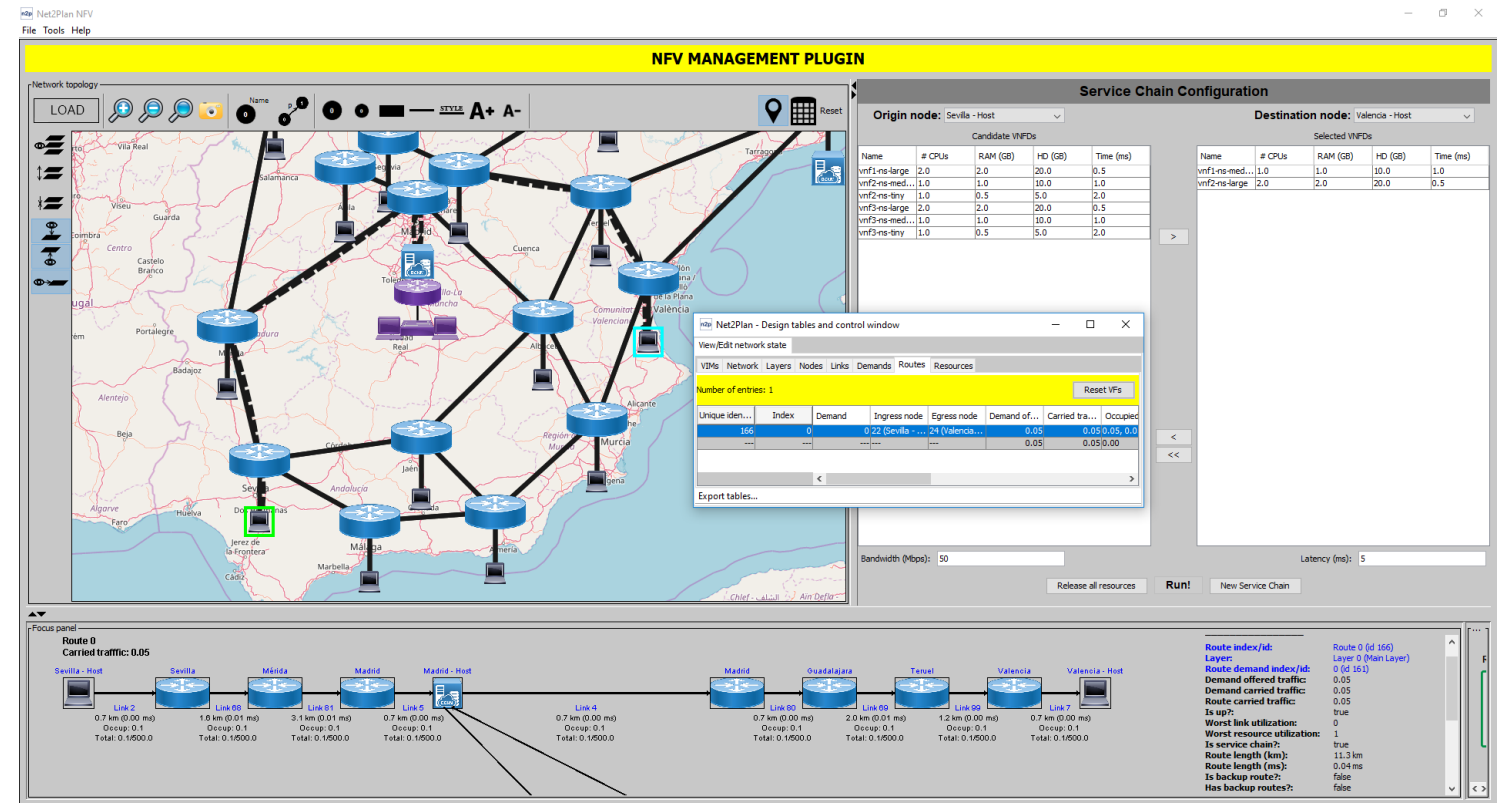

Figura 3.16: Captura de la interfaz gráfica de usuario en Net2plan. Demostración [Moren18d].

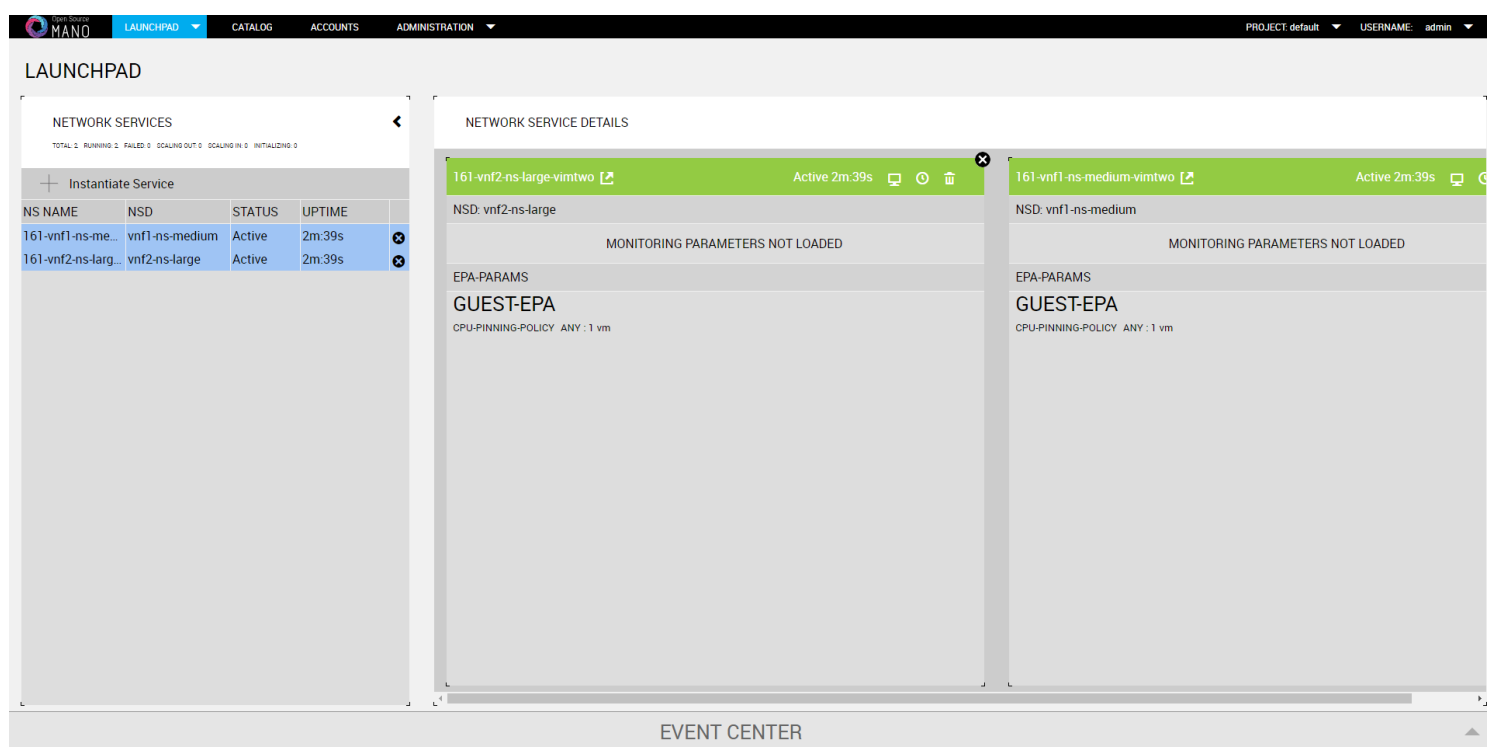

Figura 3.17: Interfaz gráfica de OSM con VNFs instanciadas. Demostración [Moren18d]. 


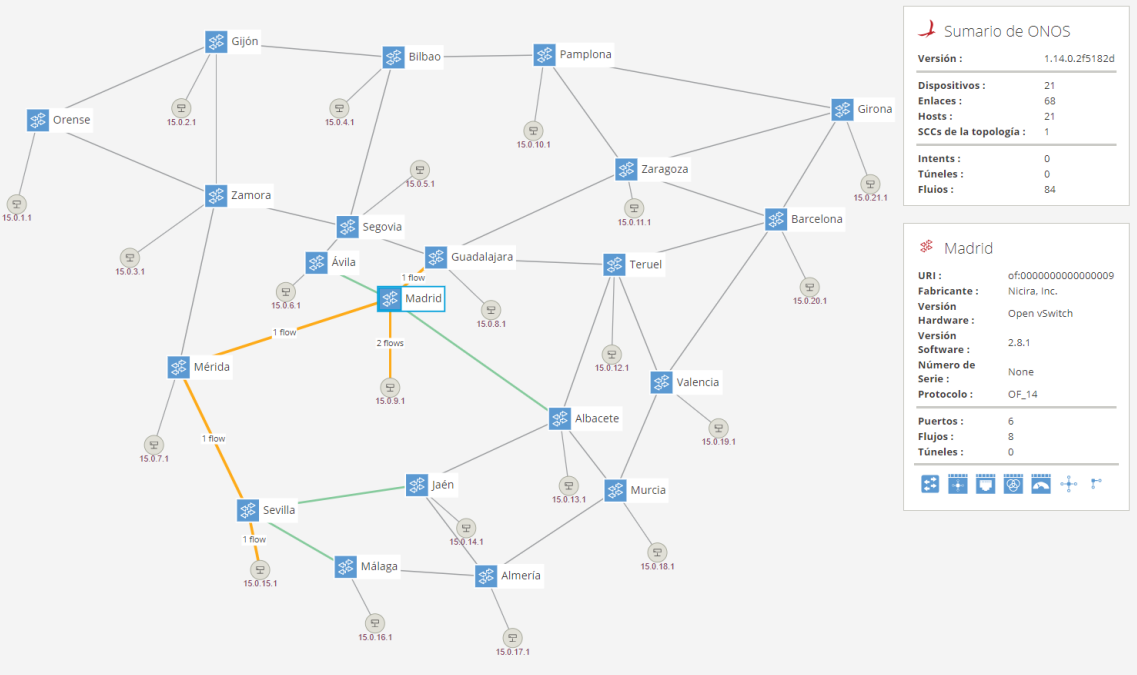

Figura 3.18: GUI de ONOS con la topología utilizada en [Moren18d] resaltando el camino seguido por el flujo.

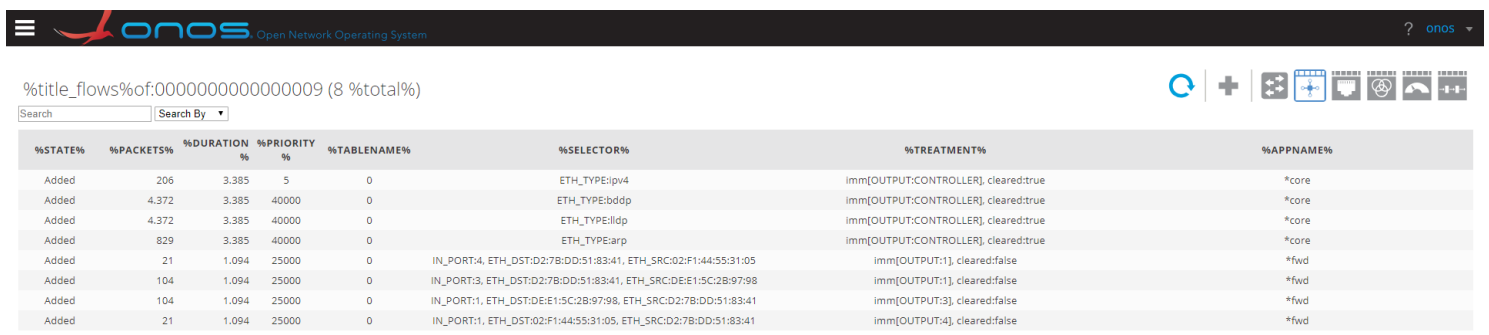

Figura 3.19: GUI de ONOS mostrando los flujos de control y datos establecidos en el sistema. 


\subsection{Conclusiones y trabajos futuros}

En esta sección se ha investigado en profundidad el paradigma de las redes controladas mediante software y la virtualización de recursos de red. Bajo tal investigación, se han propuesto una serie de casos de uso donde la optimización puede jugar un papel relevante en los escenarios de red SDN-NFV, utilizando únicamente herramientas en código abierto. Con el fin de realizar pruebas de concepto del paradigma SDN-NFV, se han presentado dos demostraciones donde queda patente, no solo la importancia de la optimización en dicho entorno, sino también los beneficios de utilizar herramientas open source. El papel de las tecnologías SDN y NFV tendrá un papel fundamental en el futuro 5G, dónde la latencia se considera como una métrica esencial. Por otro lado, el rol de las tareas de optimización en dicho contexto, pudiendo ser ejecutadas como OaaS desde la capa de aplicación, proporciona un acercamiento a la satisfacción de las exigencias 5G. Tanto la latencia, como la optimización, han sido tenidas en cuenta en el diseño de dichas pruebas conceptuales.

La líneas de trabajos futuras en referencia al campo SDN-NFV por parte del grupo de investigación, pueden ir orientadas hacia dos principales ramas, emulación y simulación de escenarios con un plano de datos complejo IP sobre WDM, basado en modelos YANG y el protocolo NETCONF, y por otro lado, la integración de VNFs con funcionalidades de red reales, pudiendo ser útiles para poder dar valor añadido a un escenario SDN-NFV mas complejo que pueda ayudar a gestionar las redes de forma eficiente, con la vista puesta en el cumplimiento de las especificaciones previstas para el escenario 5G. 



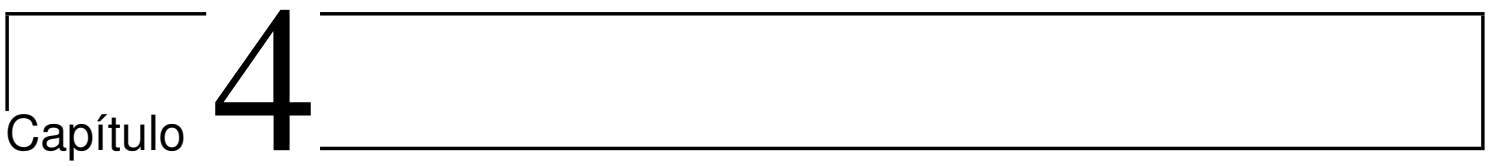

\section{Redes Flex-grid y SDM}

4.1. Aumento de la capacidad de red. Flex-grid y SDM . . . . . . . . . . . 60

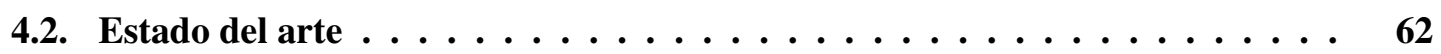

4.3. Arquitecturas de ROADM en contextos SDM y flex-grid . . . . . . . . . . 64

4.4. Análisis de escenario estático . . . . . . . . . . . . . . . 70

4.5. Análisis de escenario dinámico $\ldots \ldots \ldots \ldots \ldots \ldots \ldots$

4.6. Conclusiones .......................... 86

En este capítulo se presentan distintas estrategias en investigación para abordar el crecimiento de tráfico de Internet, desde la perspectiva de la infraestructura de red a nivel de enlace óptico. La multiplexación por división espacial o SDM (Space Division Multiplexing) se presenta como una posible solución para aportar mayor capacidad a los enlaces sin la necesidad de un aumento considerable en el tamaño de las fibras. Por otro lado, la tecnología flex-grid aporta una mejor gestión espectral basada en una mayor flexibilidad en la construcción de los canales de transmisión óptica.

El presente capítulo se centra en el análisis y evaluación de distintas alternativas de implementación para nodos ópticos en el entorno flex-grid/SDM según los diseños broadcast-and-select y route-and-select. Se propone una nueva idea conceptual en el campo SDM, llamada Space-Continuity Contraint (SCC) o restricción por continuidad espacial. Dicha idea es referida a la restricción de uso de un único espacio de multiplexación a lo largo de todo el camino de trasmisión óptica, con el que se espera aumentar la capacidad de los enlaces, mientras se disminuye la complejidad en la fabricación de conmutadores respecto de su diseño de referencia, donde no existe ningún tipo de restricción de canal de multiplexación para establecer comunicaciones ópticas entre un origen y un destino en redes WDM o elásticas. La idea general SCC tiene una particularización en el contexto MCF (Multi-Core Fiber), CCC (Core-Continuity Contraint), en cuyo caso, la restricción va limitada al uso de un core. La comparación de los diseños propuestos con su referencia flexible se realiza considerando escenarios estáticos (o de planificación previa) y dinámicos. En este último ambiente, la propuesta general SCC es comparada también dentro de la tecnología Multi Fiber (MF). 
El trabajo que se aborda en este capítulo ha sido presentado en los congresos internacionales ONDM [Rumip17a] e ICTON [Moren18c] y se ha publicado en las revistas internacionales IEEE/OSA Journal on Optical Communications and Networking [Moren17] y Elservier Computer Communications [Rumip18].

\subsection{Aumento de la capacidad de red. Flex-grid y SDM}

Las previsiones en el crecimiento del tráfico IP para los próximos años han motivado el estudio de nuevas tecnologías bajo el fin de lidiar con tales incógnitas. Una de las estrategias que ha sido objeto de gran interés por parte de la comunidad investigadora de redes ópticas ha sido la tecnología basada en la división por multiplexación de longitud de onda (Wavelength Division Multiplexing, WDM). El enfoque WDM aporta un aumento de la capacidad de las fibras mono-modo (Single Mode Fiber, SMF) en el nuevo escenario de las redes elásticas.

\subsubsection{Redes elásticas}

Las redes elásticas o flex-grid [Gerst12], ofrecen un espacio espectral mínimo, denominado como frequency slot (FS), de $12.5 \mathrm{GHz}$ o $6,25 \mathrm{GHz}$ para el establecimiento de lightpahts, según las especificaciones de la International Telecommunication Union (ITUT), mientras que las redes fijas WDM o fixed-grid [ItuT12] el FS fijo para la utilización espectral se considera de $50 \mathrm{GHz}$. La tecnología flex-grid permite transmisiones ópticas de con una tasa de bits (bit rate) muy elevada, debido principalmente a la concatenación de múltiples sub-canales adyacentes, aumentando la programabilidad de la transmisión cuando se utiliza conjuntamente con diversas posibilidades en los formatos de modulación [Lopez16]. La Figura 4.1 pone de manifiesto el uso ineficiente del espectro en redes fixed-grid respecto de la utilización mediante tecnologías elásticas.

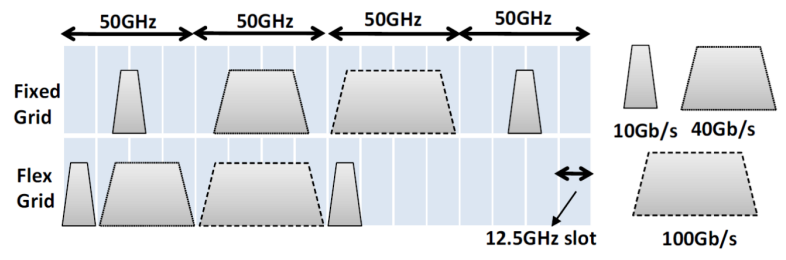

Figura 4.1: Uso espectral para tecnologías fixed-grid vs. flex-grid. Fuente [Imran18].

Esta flexibilidad aportada por las redes elásticas es soportada por un conjunto de elementos ópticos específicos, como los transpondedores de ancho de banda variable (BVT, Bandwidth Variable Transponder) [Svalu13]. Este dispositivo adapta el bit rate solicitado por la demanda de tráfico utilizando diferentes grados de flexibilidad, como baud rate, formato de modulación o número de sub-canales [Tanak13]. Normalmente, este tipo de operaciones, además de la entrada y salida de comunicaciones ópticas en los nodos, están integradas en un dispositivo mayor que es básico en las redes elásticas, el ROADM (Reconfigurable Optical Add/Drop Mulitplexer). Este dispositivo supone una evolución del 
elemento OADM (Optical Add/Drop Multiplexer)) aportando automatización a los procesos de bypass y add/drop con independencia de interacción manual externa. El elemento central del ROADM es el WSS (Wavelength Spectrum Switch) quien discrimina entre las distintas longitudes de onda disponibles para poder transmitir. La equivalencia del WSS en un ROADM para redes elásticas es el SSS (Spectrum Selective Switch), que en lugar de seleccionar por longitud de onda puede discriminar en rangos espectrales variables.

\subsubsection{Multiplexación mediante división espacial}

De forma complementaria a la tecnología flex-grid, la multiplexación mediante división espacial (SDM), se presenta como un enfoque prometedor para redes de transporte óptico. Actualmente la tecnología SDM se encuentra en estado de desarrollo con el objetivo puesto en aumentar proporcionalmente la capacidad de las actuales fibras SMF según el número de nuevos espacios físicos disponibles para la transmisión. La solución inmediata para la aplicación de SDM es unir distintas fibras mono modo en un único haz [Wizne15], esto se conoce como multi-fibra (MF), donde la multipliciadad añadida es igual al numero de SMFs en el haz. Otro enfoque que aprovecha la idea SDM, es el tipo de fibra óptica que pueda transmitir información a través de una única fibra mono core, pero en múltiples modos, MMF (Mutli-Mode Fibers). La multiplicidad viene dada por el número de modos. Existe un subconjunto de posibilidades dentro de MMF, si se utilizan únicamente unos pocos modos dentro del núcleo de la fibra se conoce como FMF (Few-Mode Fiber). Otra alternativa muy extendida es unir bajo el mismo haz diferentes núcleos mono-modo para poder expandir la capacidad en los enlaces. Este ultimo enfoque se conoce como MCF o fibras multi-core, es la tecnología que se analiza en detalle en el presente capítulo.

\subsubsection{Fibras con varios núcleos}

La fibra multi core se presenta como una de las propuestas para SDM mas populares entre la comunidad investigadora. El acoplamiento entre distintos núcleos o modos es un parámetro a considerar en las fibras basadas en SDM, a. Por un lado, la idea de agregar algunos modos a las fibras MCF se conoce como FM-MCF, sin embargo pueden presentar cierta complejidad derivada del acoplamiento entre modos y núcleos [Arik13]. Un alto grado de acoplamiento normalmente va acompañado de un aumento de la complejidad del procesamiento de la señal, siendo necesario el uso de DSP MIMO (Digital Signal Processing Multiple-Input Multiple-Output). Las fibras multi core desacopladas eliminan la dependencia de procesamiento MIMO, disminuyendo el número de correlaciones entre entradas y salidas, debido, principalmente, a su baja interferencia entre núcleos (ICXT, Inter-Core CrossTalk). El ICXT es totalmente relevante en el cálculo del alcance de transmisión (Transmission Reach) óptico de la fibra [Sakag12][SSakag14][ [Puttn17] en el contexto MCF. Este trabajo se ha centrado especialmente en este último tipo de fibras.

\subsubsection{Súper canales espaciales}

Las distintas estrategias que componen el paradigma SDM habilitan diferentes políticas o posibilidades de asignación de recursos espectrales o espaciales según el tipo de multiplexación espacial dada. Se entiende como súper canal (Super Channel, SCh), en 
un contexto flex-grid, como la composición de varios sub-canales (SbC) para realizar una transmisión óptica conjunta. La constitución de SCh depende principalmente de cómo estén considerados los sub-canales tanto en el dominio espacial como espectral. El rendimiento total de la red viene influenciado por el tamaño de las bandas de guarda (Guard Bands, GBs). Es necesario mencionar el papel de las GBs para aislar espectralmente los distintos SCh para cada espacio de multiplexación. La Figura 4.2 muestra tres tipos diferentes de formación de súper canales, (a) considerando el dominio espacial (Spa-SCh), (b) según los dominios espacial y espectral conjuntamente (S2-SCh) y (c) únicamente en el dominio espectral (Spe-SCh).

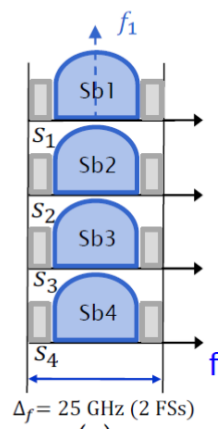

(a)

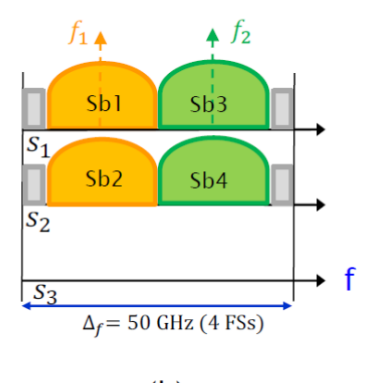

(b)

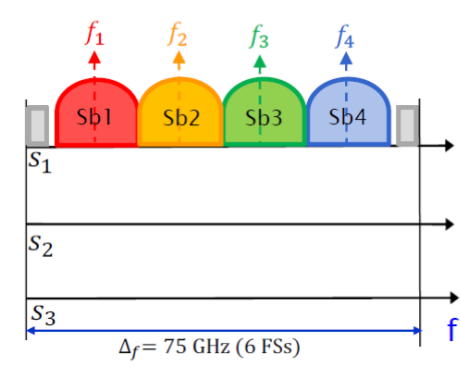

(c)

Figura 4.2: Tipos de asignación de SCh. (a) Spa-SCh, (b) S2-SCh y (c) Spe-SCh. Fuente: [Rumip18].

Como se puede apreciar en la Figura 4.2(a), se puede formar un Spa-SCh utilizando únicamente una frecuencia central a lo largo de varios espacios de multiplexación (core, fibra o modo) disponibles, además de los correspondientes GBs por cada espacio. Sin embargo, en el caso S2-SCh (4.2 (b)), los cuatro sub-canales están distribuidos en $\mathrm{S}_{1}$ y $\mathrm{S}_{2} \mathrm{y}$ necesita dos frecuencias centrales para la creación del súper canal. Nótese en este caso, que, por una parte, únicamente se está utilizando $4 \mathrm{GBs}$, la mitad respecto a el Spa-SCh, sin embargo, los recursos espectrales de $\mathrm{S}_{3}$ están totalmente desaprovechados. La formación de un Spe-SCh 4.2 (c)) presenta la mayor eficiencia espectral al tener únicamente 2 GB para los cuatro SbCs, sin embargo la utilización espacial no es la mas óptima.

\subsection{Estado del arte}

En la literatura se pueden encontrar multitud de propuestas de arquitecturas para ROADMs que se adaptan al nuevo paradigma de las redes elásticas [Colli13]. Las arquitecturas ROADM se clasificar en dos grandes diseños: broadcast-and-select (B\&S) y route-and-select (R\&S) [Garri15]. La Figura 4.3 muestra dichas arquitecturas como nodos ópticos.

El diseño broadcast-and-select (Fig. 4.3 (a)) ofrece menor coste, consumo energético y presenta menor complejidad en la parte óptica/electrónica. Sin embargo, en el bloque de entrada, la existencia de splitters pasivos puede conllevar un elevado grado de pérdidas de señal respecto de los ROADMs R\&S. Este alto grado de pérdidas podría acarrear la necesidad de amplificación interna en el nodo, aumentando el coste. Por otro lado, los 

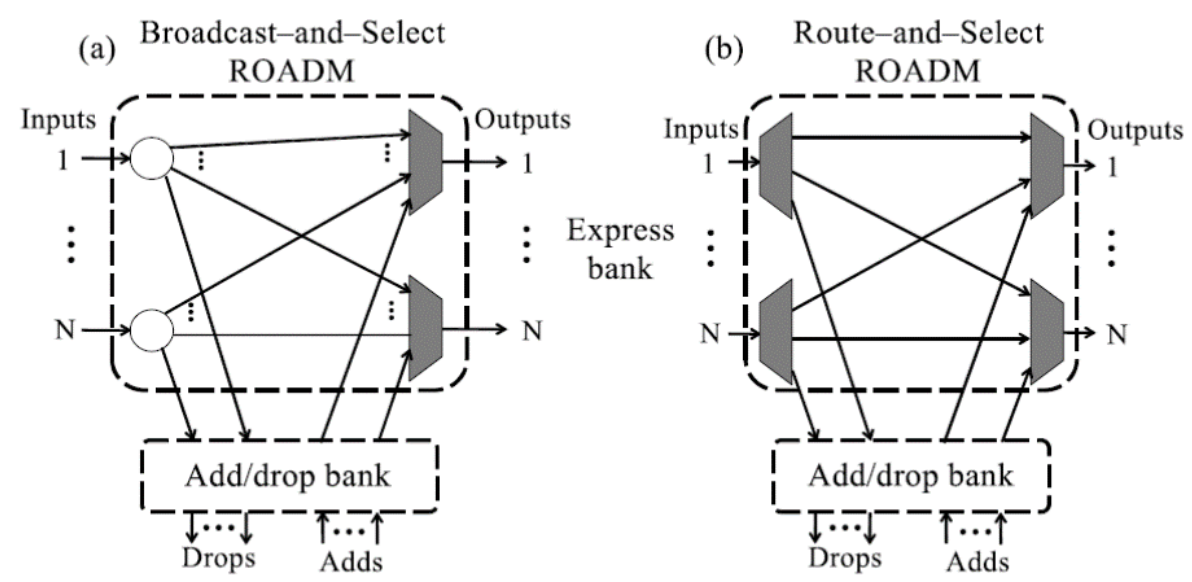

Figura 4.3: Arquitecturas de nodo óptico: (a) Broadcast-and-select y (b) Route-and-select. Fuente: [Garri15].

diseños route-and-select, ofrecen mayor aislamiento respecto del bloqueo en los puertos. Esto es debido al aumento del número de SSSs, que además implica un menor grado de pérdidas sin importar el número de puertos [Filer14]. R\&S puede verse como una variación de B\&S, donde en el módulo de entrada presenta SSSs para discriminar entre longitudes de onda en lugar de splitters, como ocurre en B\&S. Un splitter óptico es un dispositivo que reparte la señal de entrada a todas las salidas disponibles, sin realizar discriminación en los puertos de salida. Según los autores de [Filer14], la arquitectura de nodo óptico B\&S se postula como la mejor opción para los ROADMs con un grado nodal $(F)$ menor o igual a 9 mientras que puede resultar favorable el uso de diseños basados en route-and-select en aquellos casos con un grado nodal superior a 9 compensando los beneficios al aumento del coste.

Las propiedades colorless, directionless y contentionless (CDC) hacen referencia a la capacidad de los nodos ópticos de redirigir señales sin restricciones de longitud de onda, dirección o contención interna. Los ROADMs que integran estas capacidades permiten un escenario sin bloqueo alguno, denominados en este trabajo como FNB-ROADMs (Fully Non-Blocking ROADM). Cabe mencionar que el aumento de capacidades tiene implicación directa en la complejidad de diseño, así como en un aumento del coste total del dispositivo. Dentro de un contexto que considere fibras multi-core, la extensión de las propiedades CDC a ROADMs implica un aumento de la complejidad considerable, siendo necesario un profundo análisis del balance rendimiento/coste en la implementación de los nodos ópticos para obtener una correcta planificación de la red. Algunos autores han investigado posibles arquitecturas en SDM-ROADM, asumiendo capacidades FNB [Muham15][Perel16b]. Los resultados de este aporte apuntan a un gran aumento de la complejidad en entornos SDM respecto de los tradicionales SMF. Para la implementación de los diseños B\&S y R\&S en ROADMs FNB, se asume un número de canales espaciales (núcleos, fibras o modos) $S$, donde el número de dispositivos SSS se incrementa según un factor $S$, mientras que el tamaño individual de cada SSS es multiplicado de nuevo por $S$, resultando un aumento de la complejidad en $S^{2}$. Este elevado factor aleja la posibilidad de implementación factible para escenarios con altos grados espaciales. 
Con el fin de simplificar tales diseños, la comunidad investigadora ha propuesto algunas arquitecturas que ofrecen un grado de complejidad menor, traducido a un menor coste, pero con una hipotética disminución en el rendimiento. La primera propuesta se conoce como arquitectura bajo demanda o Architecture on Demand (AoD) [Amaya13], orientada a eliminar los módulos físicos infrautilizados compartiendo recursos bajo demanda expresa gracias una gran capacidad de programación. En este diseño, todos los componentes de hardware, como puertos de entrada/salida, módulos add/drop, SSSs, combinadores de señal, amplificadores y (de-)multiplexores están conectados a un plano común donde las conexiones se pueden realizar ad-hoc según la demanda de tráfico dada. Por otro lado, la arquitectura de nodo Joint-switching (JoS) [Nelso14], ofrece una comparativa reducción en el número de SSSs, conmutando de forma simultánea las señales provenientes de todos los núcleos en un puerto, aumentando el número de puertos en los SSSs, lo que implica un mayor coste unitario de los mismos. Esta opción es recomendada para fibras SDM con un alto grado de acoplamiento, por lo que un postprocesado MIMO es completamente necesario para discriminar la información y permitir un establecimiento de comunicación end-to-end de forma apropiada. Otro enfoque para el diseño de nodos ópticos en escenarios SDM trata de reducir la complejidad de hardware respecto de JoS, en los trabajos [Marom15] y [Khoda16] es denominado como Independent-Switching (InS). Este diseño analiza los nodos origen y destino de las demandas para compartir recursos espectrales, de esta manera creando cierta independencia del resto de lightpaths que atraviesan el nodo. En [Marom15] se propone una arquitectura intermedia entre IoS y JoS, llamada Fractional $J o S$, donde es posible agrupar varios canales espaciales para aportar mayor flexibilidad. Las arquitecturas basadas en Swithcing se analizan y comparan desde una perspectiva de coste y complejidad de nodo en el artículo [Marom17].

En este capítulo se han utilizado como comparación y validación de la propuesta SCC SDM-ROADM, las arquitecturas FNB y JoS bajo el enfoque route-and-select. Estos tres diseños se exponen, detallan y comparan en la siguiente sección.

\subsection{Arquitecturas de ROADM en contextos SDM y flex- grid}

El elemento reconfigurable OADM se encarga de las operaciones de entrada $(a d d)$, salida (drop) y bypass en los lightpaths para un determinado nodo óptico. El dispositivo principal en los flex-grid-ROADM es el SSS, quien es capaz de alternar entre cualquier slot de frecuencia a cualquiera de los puertos disponibles en el nodo, se trata de la evolución a redes elásticas del dispositivo WSS (Wavelength Selective Switch). Sin embargo, su uso en el diseño de ROADM presenta ciertas restricciones, debido a que es el elemento con mayor coste en un nodo óptico [Ruiz14]. Como se ha expuesto en la anterior sección, existen dos enfoques principales para el diseño de ROADMs en escenarios elásticos, B\&S y R\&S. En el trabajo presentado en [Moren17], se presenta bajo el diseño Broadcast-andSelect para redes elásticas, una implementación de un ROADM tipo FNB que sirve como referencia a la propuesta de nodo óptico denominada como Core-Continuity-Constaint o CCC-ROADM. En este trabajo se muestra un breve análisis de las pérdidas introducidas por los splitters (proporcional a $\log (Y)$ en dBs, siendo $Y$ el número de salidas del dispo- 
sitivo) para cada arquitectura. Puesto que el trabajo expuesto en [Rumip18] se centra en mayor medida en el rendimiento de los ROADMs en un escenario dinámico, dejando fuera del estudio las pérdidas por dispositivos pasivos y el bloqueo interno, se ha elegido el enfoque Route-and-Select para los ROADMs FNB, JoS y SCC (Spatial-Continuity-Constraint) en el contexto de redes flex-grid. Nótese que un diseño ROADM B\&S se puede convertir en un R\&S únicamente intercambiando en los módulos de entrada los splitters por dispositivos SSS. Este hecho, además de la evasión de redundancia en el capítulo, han motivado que las figuras expuestas en esta sección sean únicamente las correspondientes al diseño route-and-select.

\subsubsection{Fully-Non-Blocking ROADM}

El diseño Fully-Non-Blocking para ROADMs proporciona una interconexión completa entre los puertos entrada y salida disponibles en el nodo óptico. En la Figura 4.4 se muestra una representación donde se ha añadido un módulo add/drop con dispositivos con propiedades CDC bajo el fin de eliminar el bloqueo interno. En la Figura 4.4 el proceso de establecimiento de nuevos lighpaths en la red está representado en color azul, mientras que, en caso de ser tal nodo el destino final, las líneas marcadas en rojo siguen el proceso de drop. El diseño FNB es el que presenta mayor rendimiento teórico, ya que no muestra limitaciones en la asignación de longitud de onda, puerto, o canal espacial, además, es posible crear súper canales de los tres tipos anteriormente descritos. En contraposición, requiere de una implementación con una complejidad considerable, siendo el coste hipotético muy alto. Este hecho se debe a que requiere una gran cantidad de dispositivos SSS, concretamente dos por cada canal espacial y grado.

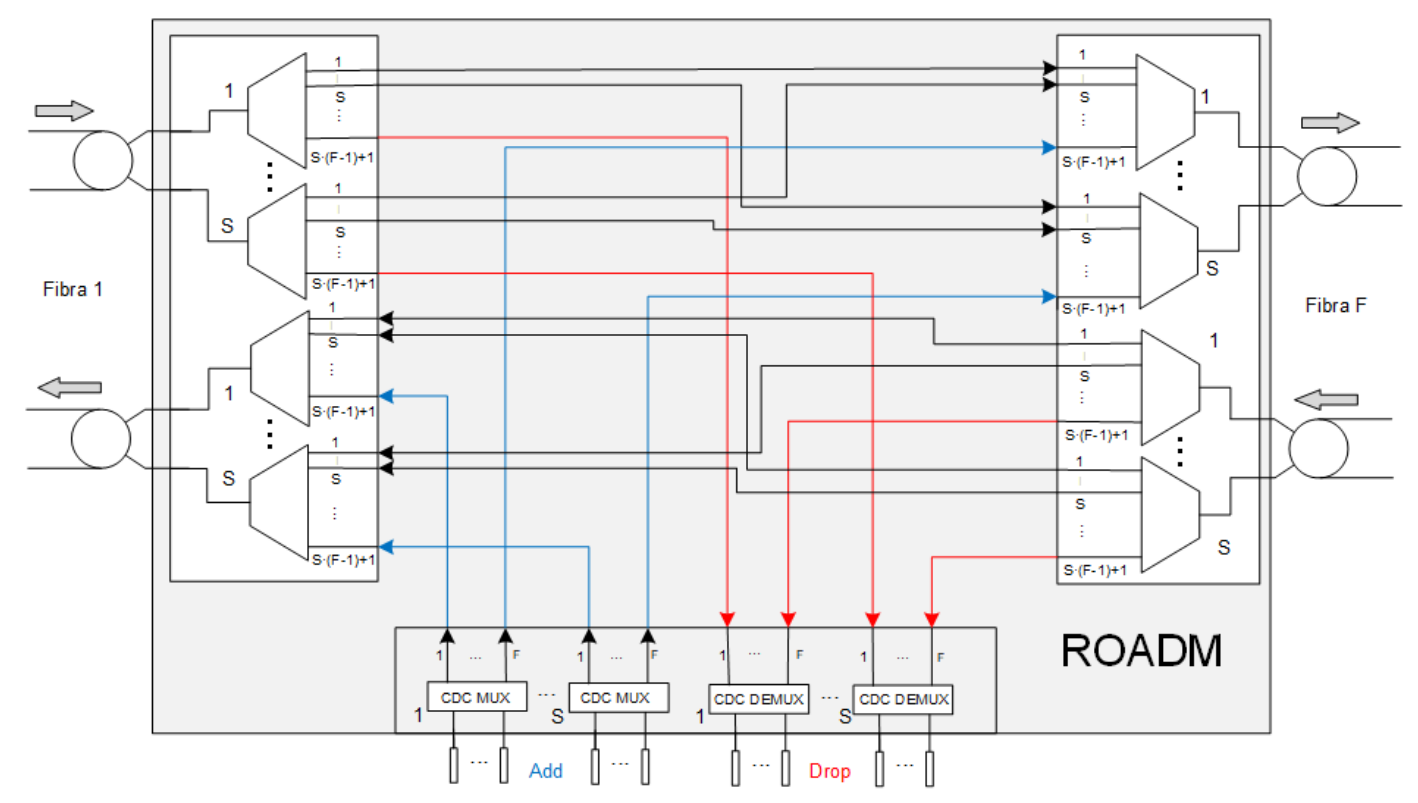

Figura 4.4: Arquitectura de nodo óptico tipo R\&S Fully-Non-Blocking 


\subsubsection{Joint-Switching ROADM}

La propuesta de Joint-Switching como nodo óptico se presenta como una alternativa de menor coste respecto de la estrategia FNB. El diseño JoS permite conmutar un rango espectral en todos los canales espaciales al mismo tiempo. El uso de SCh en la arquitectura JoS queda restringido únicamente a Spa-SCh. Este factor condiciona el número de SSSs necesarios en la implementación reduciéndose el número de SSSs a únicamente dos por puerto, como se puede ver en la Figura 4.5. Sin embargo, requiere el uso de SSS con un tamaño $S x S \cdot F$, siendo $S$ el número de canales espaciales y $F$ el número de puertos de entrada/salida en el nodo. Este elevado número de puertos en los SSSs necesarios puede causar problemas a la hora de encontrar dispositivos SSS comerciales fabricados con un alto grado nodal o canales espaciales.

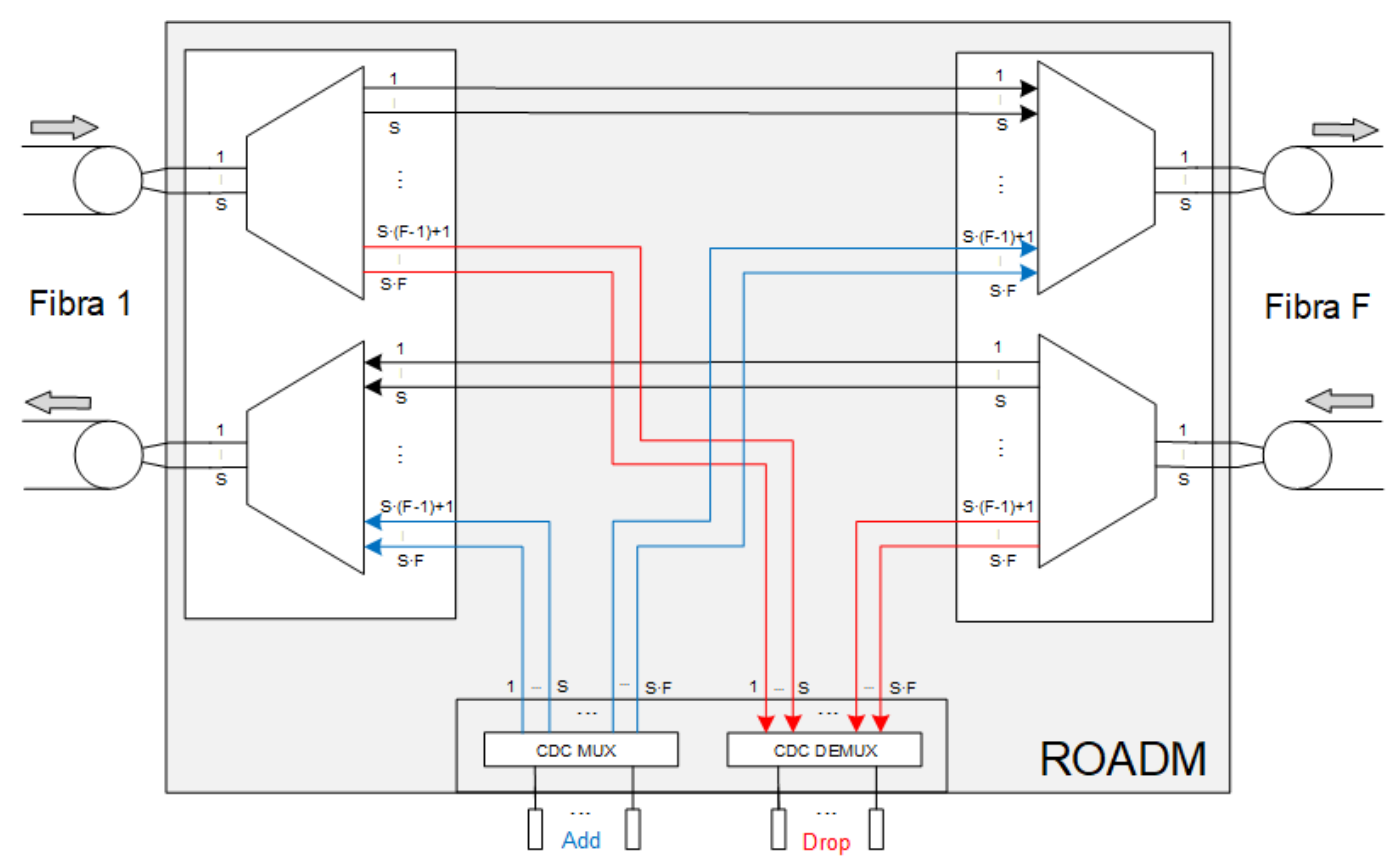

Figura 4.5: Arquitectura de nodo óptico tipo R\&S Joint-Swithcing

\subsubsection{Spatial-Continuity-Constraint ROADM}

Otra forma de introducir simplicidad al diseño en comparación con FNB, es restringir el routing a un único canal de multiplexación desde el origen hasta el destino. Esta idea es referida como Core-Continuity-Constraint (CCC) en [Rumip17a], [Moren17] y [Moren18c], con aplicaciones únicas en contextos de MCF. Por otra parte, la extensión de este enfoque a todos los tipo canales espaciales (SDM) se ha denominado como SpatialContinuity-Constraint (SCC) en [Rumip18]. Por otro lado, en el trabajo [Marom17] esta idea es referida como routing without spatial lane change, literalmente, encaminamiento sin cambio de canal espacial. En lo referente a esta parte del trabajo esta idea se mencionará como Spatial-Continuity-Contraint o SCC. La implementación propuesta para un nodo óptico basado en SCC SDM-ROADMs se expone en la Figura 4.6. 


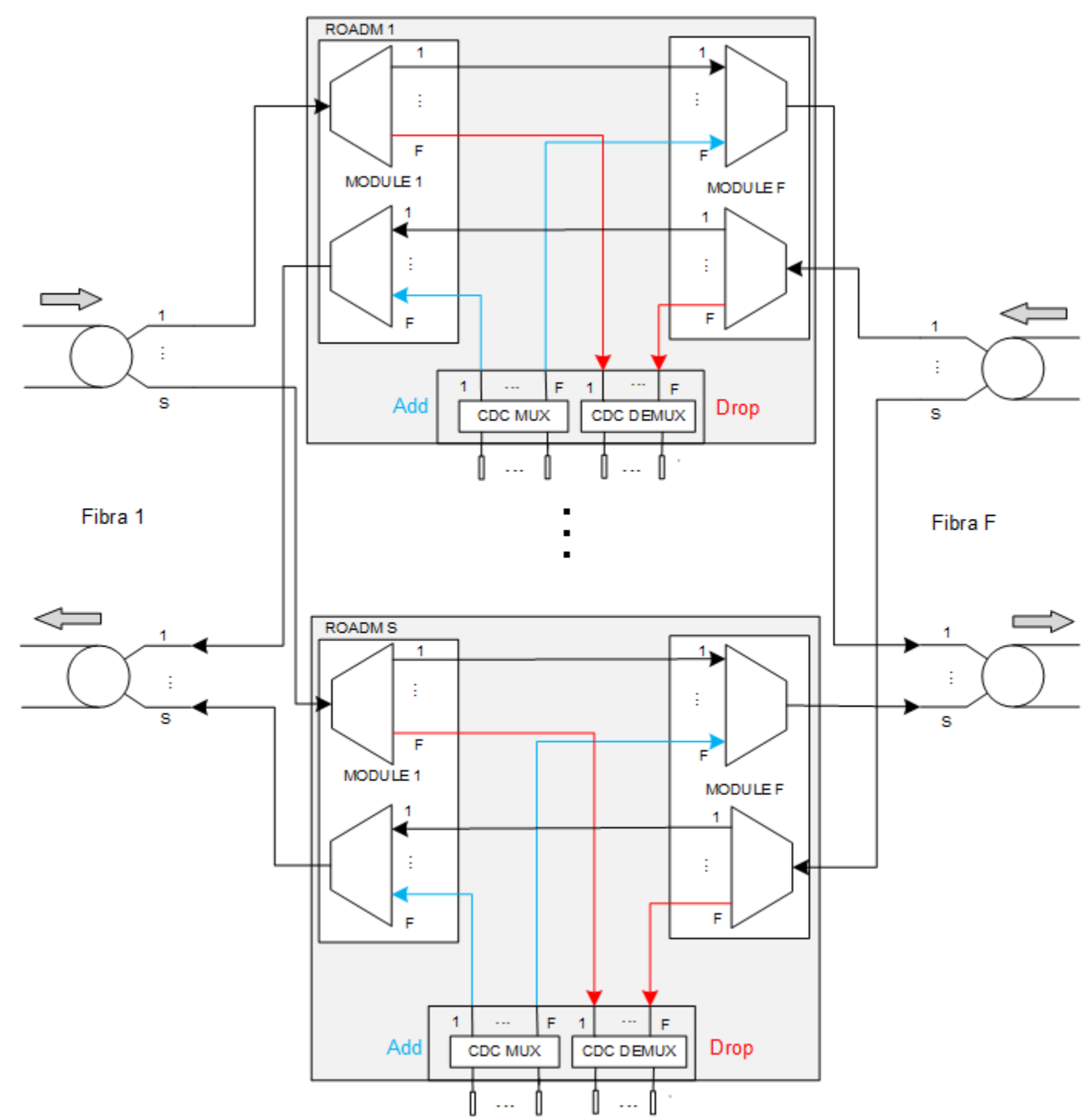

Figura 4.6: Arquitectura de nodo óptico tipo Spatial-Continuity-Constraint

\subsubsection{Comparación de arquitecturas}

En esta parte de la sección se comparan las implementaciones propuestas para nodos ópticos en [Moren17] (B\&S) y en [Rumip18] (R\&S). Ambos análisis dan uso a las figuras esquemáticas anteriormente descritas bajo el diseño route-and-select. La aplicación a B\&S se puede realizar sin mas que sustituir los SSSs correspondientes a los módulos de entrada en los ROADMs por splitters pasivos en cada arquitectura presentada anteriormente para $R \& S$.

\subsubsection{Broadcast-and-Select. FNB vs. CCC}

Algunas características relevantes de la implementación del nodo óptico, sin restricciones de bloqueo (FNB), para redes elásticas y fibras MCF, que se propone en la Figura 4.4 bajo el contexto B\&S (splitters en lugar de SSS en las entradas), se exponen a conti- 
nuación:

- El nodo óptico está implementado en un único ROADM.

- El número total de splitters y SSSs es $F \times C$, demostrando una dependencia lineal con el número de fibras $(F)$ y de núcleos $(C)$.

- El número de puertos de salida en los SSSs es $(F-1) C+1$, linealmente proporcional el número de cores y fibras.

- Las pérdidas de señal como consecuencia del uso de splitters son $10 \cdot \log [(F-) C+1]$, es decir, las pérdidas, en $\mathrm{dB}$, tienen dependencia logarítmica con el número de fibras y núcleos.

- El número total de dispositivos CDC MUX/DEMUX en el módulo add/drop es $C$, es decir un dispositivo por cada core.

Por otro lado, el diseño propuesto para implementar un nodo óptico flex-grid/MCF B\&S CCC-ROADM con grado nodal $F$, fibras de $C$ núcleos y módulo add/drop, se basa en la Figura 4.6 realizando los cambios correspondientes en los módulos de entrada. Sus principales características son:

- La arquitectura puede ser construida como una integración en paralelo de ROADMs SMF tradicionales de grado $F$, uno por cada núcleo.

- El número total de dispositivos splitters y SSSs es $F$ y es independiente del número de núcleos de en cada fibra.

- El número de puertos de cada SSS es $F$, tiene dependencia lineal única con el número de fibras (grado nodal).

- Las pérdidas introducidas por los splitters crecen con dependencia logarítmica según el número total de fibras, $10 \cdot \log (F)$. Independencia del número de cores.

- Un CDC MUX y CDC DEMUX de tamaño $1 \mathrm{x} F$ por cada ROADM en el puerto de add/drop.

Las tablas 4.1 y 4.2 ofrecen un análisis entre los diseños B\&S FNB y B\&S CCC comparando para cada caso el nivel de pérdidas en los splitters y el tamaño en los puertos de los SSSs respectivamente, para tres valores de grado nodal y tres tipos de fibras MCF distintas.

La tabla 4.1 muestra que las pérdidas debido a los splitters en enfoques B\&S afectan de manera considerable a los nodos ópticos FNB. La dependencia de tales valores con el número de núcleos en las fibras puede implicar la utilización de amplificación interna. Suponiendo necesaria la amplificación para pérdidas mayores a $20 \mathrm{~dB}$, según el diseño FNB, se requiere del uso de amplificadores para un contexto de fibras de, al menos, 19 núcleos y un nodo con 8 fibras o mayor, mientras que en el caso CCC, la amplificación sería necesaria para un número de fibras mayor a 100, independientemente del número de núcleos de la fibra. 
Tabla 4.1: Pérdidas introducidas por los splitters [dB].

\begin{tabular}{c|cccccc}
\hline \multirow{2}{*}{$\boldsymbol{C}$} & \multicolumn{2}{|c}{$\boldsymbol{F = \mathbf { 2 }}$} & \multicolumn{2}{c}{$\boldsymbol{F}=\mathbf{4}$} & \multicolumn{2}{c}{$\boldsymbol{F}=\mathbf{8}$} \\
\cline { 2 - 7 } & FNB & CCC & FNB & CCC & FNB & CCC \\
\hline $\mathbf{7}$ & 9,03 & 4,77 & 13,42 & 6,99 & 16,99 & 9,54 \\
$\mathbf{1 2}$ & 11,14 & 4,77 & 15,68 & 6,99 & 19,29 & 9,54 \\
$\mathbf{1 9}$ & 13,01 & 4,77 & 17,63 & 6,99 & $\mathbf{2 1 , 2 7}$ & 9,54 \\
\hline
\end{tabular}

Tabla 4.2: Número de puertos de entrada por SSS

\begin{tabular}{c|cccccc}
\hline \multirow{2}{*}{$\boldsymbol{C}$} & \multicolumn{2}{|c}{$\boldsymbol{F}=\mathbf{2}$} & \multicolumn{2}{c}{$\boldsymbol{F}=\mathbf{4}$} & \multicolumn{2}{c}{$\boldsymbol{F}=\mathbf{8}$} \\
\cline { 2 - 7 } & FNB & CCC & FNB & CCC & FNB & CCC \\
\hline $\mathbf{7}$ & 8 & 2 & 22 & 4 & $\mathbf{5 0}$ & 8 \\
$\mathbf{1 2}$ & 13 & 2 & $\mathbf{3 7}$ & 4 & $\mathbf{8 5}$ & 8 \\
$\mathbf{1 9}$ & 20 & 2 & $\mathbf{5 8}$ & 4 & $\mathbf{1 3 4}$ & 8 \\
\hline
\end{tabular}

Por otro lado, se puede apreciar en la tabla 4.2 el tamaño de los dispositivos SSS para las implementaciones de nodos basadas en B\&S y MCF. El tamaño máximo para puertos en SSSs encontrado en la literatura, a fecha de redacción de este trabajo, es de 1x32 [Lument17]. En la tabla están resaltados en negrita aquellas configuraciones que superan el tamaño 1x32. En los nodos FNB, para fibras MCF resultaría muy complicado encontrar SSSs comerciales en caso de utilizar fibras con un alto número de cores o de fibras totales en el nodo. Sin embargo, para la opción CCC las limitaciones por tamaño empezarían únicamente para nodos con un número de fibras superior a 32 , hecho inusual en un diseño de redes de transporte ópticas tradicional.

\subsubsection{Route-and-Select. FNB vs. JoS vs. SCC}

La tabla 4.3 resume un análisis de varios nodos ópticos dentro de un contexto de redes elásticas SDM basados en los diseños route-and-select de las figuras 4.4. 4.5 y 4.6. El primer punto a destacar es el beneficio en menor coste que aporta el diseño JoS en cuanto a número de SSSs por grado, únicamente se necesitan dos, respecto al las arquitecturas FNB y SCC que son proporcionales al número de canales de multiplexación espacial $(S)$. Por otro lado, la opción FNB, se apunta como la de mayor rendimiento teórico, pero a costa un gran tamaño de SSS, en algunos casos, como se puedo apreciar en la tabla 4.3 , mucho mayor al propio de los disponibles comercialmente [Lument17].

Tabla 4.3: Métricas de nodo óptico para diferentes diseños SDM-ROADM

\begin{tabular}{|c|c|c|c|c|}
\hline SDM-ROADM & \# SSSs por grado & \# Puertos SSS & Tamaño SSS & Tipos SCh \\
\hline FNB & $2 \cdot S$ & $S \cdot(F-1)+2$ & $1 \times[S \cdot(F-1)+1]$ & Todos \\
\hline JoS & 2 & $S+S \cdot F$ & $S \times(1 \times F)$ & Spa-SCh \\
\hline SCC & $2 \cdot S$ & $F+1$ & $1 \times F$ & Todos \\
\hline
\end{tabular}

En el aspecto de tamaño de los SSSs, el diseño SCC se postula como la mejor opción 
debido a la independencia de $S$ en el propio tamaño, a diferencia de las propuestas FNB y JoS. Finalmente, desde el punto de vista de la versatilidad de uso de diferentes tipos de SChs, los ROADMs diseñados bajo premisas de Joint-Switching únicamente pueden utilizar súper canales espaciales mientras que para SCC y FNB que pueden gestionar comunicaciones utilizando todos los SChs vistos en este capítulo.

\subsection{Análisis de escenario estático}

En esta sección se presenta una descripción del escenario estático (offline) bajo condiciones de redes flex-grid y con fibras MCF utilizado en el trabajo [Moren17], así como la presentación de resultados obtenidos y discusión tras la ejecución de las simulaciones. Para evaluar la propuesta de diseño de nodos ópticos Core-Continuity-Constraint frente a la referencia FNB, se han desarrollado dos algoritmos que tienen como objetivo resolver el problema de asignación de camino, modulación, espectro y núcleo (Routing, Modulation, Spectrum and Core Assignment, RMSCA) bajo el contexto flex-grid/MCF según los enfoques FNB y CCC.

\subsubsection{Algoritmos}

El primer algoritmo es una implementación basada en una formulación ILP y fue presentado en [Rumip17a] y utilizado en [Moren17] para un escenario estático, mientras que el algoritmo restante se trata de un método heurístico para resolver el RMSCA bajo el mismo entorno. Este último enfoque está publicado en [Moren17].

\subsubsection{Algoritmo ILP}

Este primer algoritmo está basado en una formulación ILP cuyo objetivo es minimizar la ocupación espectral total en la red, es decir, minimizar el número total de FSs utilizado por todos los cores de todas las fibras de la red mientras se optimiza el tráfico total llevado. Este ILP considera variaciones que modelan el comportamiento tanto para ROADMs FNB como CCC dentro del contexto flex-grid/MCF. Los parámetros de entrada comunes a ambos enfoques se expone como sigue:

- $\mathcal{N}$ : Conjunto de nodos en la red.

- $\mathcal{E}$ : Conjunto de enlaces de la red.

- $\mathcal{C}$ : Conjunto de núcleos de las fibras $\mathrm{MCF}$, tal que $|\mathcal{C}|=C$.

- $\mathcal{D}$ : Conjunto total de las demandas de tráfico.

- $\mathcal{T}$ : Conjunto total de tranponders disponibles.

- $\mathcal{S}$ : Conjunto total del slots en cada núcleo.

- $h_{d}, d \in \mathcal{D}$ : Tráfico ofrecido por la demanda $d$, medido en Gbps, pudiendo ser satisfecho por varios lightpahts. 
- $\mathcal{P}_{d t}, d \in \mathcal{D}, t \in \mathcal{T}$ : Para cada demanda $d$ y tipo de transponder $t$, lista de caminos admisibles mas cortos entre los nodos origen y destino de la demanda $d$ que cumplan el alcance óptico de transmisión del transponder $t$.

- $\mathcal{P}$ : Conjunto total de caminos admisibles en la red, de tal manera que $\mathcal{P}=\cup_{d \in \mathcal{D}, t \in \mathcal{T}}$ Se considera $\mathcal{P}_{e}$ al conjunto de caminos que atraviesan el enlace $e$. Dado un camino $p, d(p)$ representa la cantidad de tráfico de la demanda $d$ que lleva el camino $p, l(p)$ denota el número total de saltos asociados a $p, t(p)$ es el transpoder utilizado en $p$. Para simplificar, se considera $r_{p}=r(t(p))$ al line rate del transponder $t$, mientras que $s_{p}=s(t(p))$ es el número de FSs necesario.

- $\mathcal{S}\left(p, s_{0}\right)$ : conjunto de slots ocupados por el camino $p$, cuando el FS inicial es $s_{0}$.

\section{Formulación ILP para RMSCA con FNB-ROADM}

La versión del ILP para resolución de RMSCA según el criterio FNB define como variables de decisión:

- $x_{p s}, \forall p \in \mathcal{P}, s \in \mathcal{S}$ : con valor 1 si el camino $p$ tiene como primer slot $s$ (el resto de slots se asumen contiguos y consecutivos) y 0 en caso contrario.

La función objetivo minimiza el número total de slots utilizados en la red:

$$
\min \sum_{p \in \mathcal{P}, s \in \mathcal{S}} s_{p} l(p) x_{p s} \quad \text { sujeto a: }
$$

un conjunto de restricciones proporcionadas por las ecuaciones:

$$
\begin{gathered}
\sum_{p \in \mathcal{P}_{d}, s \in \mathcal{S}} r_{p} x_{p s} \geq h_{d}, \quad \forall d \in \mathcal{D} \\
\sum_{p \in \mathcal{P}_{e}, s \in \mathcal{S}\left(p, s_{0}\right)} x_{p s_{0}} \leq C, \quad \forall e \in \mathcal{E}, s \in \mathcal{S}
\end{gathered}
$$

La ecuación (4.2) asegura el cumplimiento de las demandas de tráfico, mientras que la expresión (4.3) impide la colisión entre los espacios espectrales, de tal manera que un FS $s$ solo puede usarse por $C$ lightpaths, uno por cada core del enlace $e$.

\section{Formulación ILP para RMSCA con CCC-ROADM}

La modificación de la formulación del ILP para la resolución de RMSCA bajo restricciones de núcleo tiene como variables de decisión:

- $x_{p c s}, \forall p \in \mathcal{P}, c \in \mathcal{C}, s \in \mathcal{S}$ : con valor 1 si el camino $p$ tiene como primer slot $s$ (el resto de slots se asumen contiguos y consecutivos) y va servido por el núcleo $c$ y 0 en caso contrario. 
La función objetivo minimiza el número total de slots utilizados en la red:

$$
\min \sum_{p \in \mathcal{P}_{d}, c \in \mathcal{C}, s \in \mathcal{S}} s_{p} l(p) x_{p c s} \quad \text { sujeto a: }
$$

restricciones dadas por las siguientes ecuaciones:

$$
\begin{gathered}
\sum_{p \in \mathcal{P}, c \in \mathcal{C}, s \in \mathcal{S}} r_{p} x_{p c s} \geq h_{d}, \quad \forall d \in \mathcal{D} \\
\sum_{p \in \mathcal{P}_{e}, s \in \mathcal{S}\left(p, s_{0}\right)} x_{p c s_{0}} \leq 1, \quad \forall e \in \mathcal{E}, c \in \mathcal{C}, s \in \mathcal{S}
\end{gathered}
$$

De forma similar a la versión para FNB, la ecuación (4.5) asegura el cumplimiento de las demandas de tráfico bajo la restricción de núcleo, mientras que (4.6) impide la colisión entre los espacios espectrales, de tal manera que un FS $s$ solo puede usarse por un lightpath por cada core $c$ del enlace $e$.

\subsubsection{Algoritmo Heurístico}

En esta parte de la subsección de algoritmia para redes flex-grid/MCF en escenarios estáticos, se expone y detalla el algoritmo heurístico para la resolución del problema RMSCA, tanto para ROADMs FNB como CCC, utilizado en el trabajo [Moren17]. La Figura 4.7 representa el pseudocódigo de tal algoritmo.

De manera análoga al algoritmo basado en el ILP expuesto anteriormente, el heurístico tiene el objetivo de maximizar el tráfico total llevado por la red mientras trata de satisfacer las demandas de tráfico bajo previo conocimiento, dentro de un contexto offli$n e$ en redes elásticas MCF. El presente heurístico está basado en la técnica de búsqueda local [Pavon16], dónde gracias a una métrica de rendimiento se evalúa de forma iterativa dentro de un bucle principal las distintas opciones del espacio de soluciones a considerar. La ejecución del algoritmo se puede dividir en dos grandes partes, rutinas fuera del bucle principal y dentro del bucle.

1. En primer lugar, es necesario pre-calcular los $k$ caminos mas cortos para cada una de las demandas de tráfico, para cada transponder disponible y son almacenadas en el conjunto $\mathcal{C P} \mathcal{L}$. Nótese que en este primer paso no se tiene en cuenta la longitud de los caminos. Por consiguiente, debe ser creado un nuevo conjunto, $\mathcal{F} \mathcal{P} \mathcal{L}$, donde se almacenen, para cada demanda $d$, los únicos caminos factibles para realizar la transmisión, es decir, cuya longitud sea soportada por el alcance óptico del transponder y modulación correspondientes.

2. El bucle principal itera a lo largo de las demandas ordenadas según el porcentaje de tráfico no cursado en orden descendente, por tanto, aquella demanda que proporcionalmente a su tamaño tenga mayor cantidad de tráfico sin satisfacer, será evaluada primero. El bucle principal finalizará cuando todas las demandas estén satisfechas, o no se pueda cursar todo el tráfico de al menos una demanda, en tal caso se considera como el fin de la ejecución. Cada iteración del bucle recalcula el tráfico restante sin cursar para cada demanda y tales demandas son almacenadas de forma ordenada 


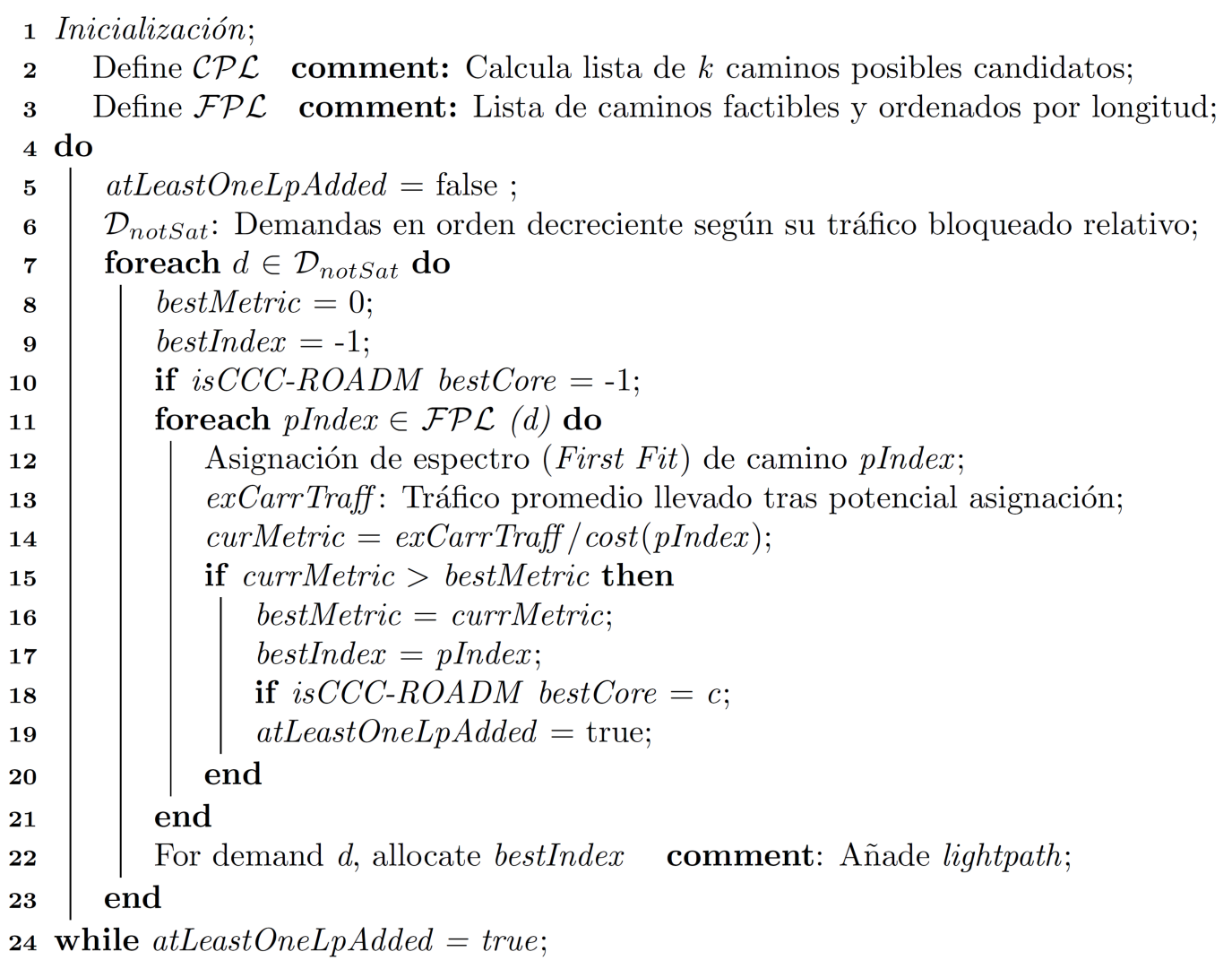

Figura 4.7: Pseudocódigo del algortimo heurístico para resolver el RMSCA.

en la lista $\mathcal{D}_{\text {notSat }}$. A continuación, para cada demanda, se itera entre las distintas posibilidades para establecer un lightpath para cada camino $p$ según una métrica de rendimiento. Tal métrica (línea 14), evalúa el tráfico total cursado en la red tras el hipotético establecimiento del lightpath a lo largo del camino mas corto $p$, teniendo en cuenta el coste espectral. Por ejemplo, si una opción da un tráfico total de 400 Gbps pero necesita 4 FSs según su transponder, tendrá una métrica igual a 100, mientras que otra opción establece un tráfico de 100 Gpbs ocupando 3 FSs, tendrá métrica con valor 33, la opción de mayor métrica es la elegida. La asignación de recursos espectrales contiguos para cada lightpath se asume como first fit.

- En caso de ser necesaria la continuidad del núcleo en la transmisión (CCC), se restringe el establecimiento de lightpath solo para aquellos caminos que dispongan de recursos espectrales libres a lo largo del mismo core $c$.

- Sin embargo, la opción sin restricción de core, FNB puede utilizar todas las posibilidades de $\mathcal{F} \mathcal{P} \mathcal{L}(d)$.

\subsubsection{Descripción del escenario estático y resultados}

En esta subsección se describe el escenario diseñado para llevar a cabo las simulaciones ejecutadas en un contexto estático de una red de transporte elástica con fibras MCF, con el fin de evaluar la propuesta de CCC B\&S MCF-ROADM frente el diseño FNB 
B\&S MCF-ROADM, así como los resultados numéricos obtenidos y una discusión sobre los mismos. Las simulaciones se engloban en dos grandes bloques, la validación del rendimiento del algoritmo heurístico respecto de su referencia en el ILP aplicada en dos topologías diferentes, y un análisis de rendimiento utilizando únicamente el heurístico para FNB y $\mathrm{CCC}$ en cuatro redes de trasporte con diferentes naturalezas (numero de nodos, tamaño).

Existe un marco común en la configuración del escenario para ambos estudios. Las ejecuciones se han implementado en la herramienta de planificación de redes Net2plan [Pavon15][Net2P] el cual incluye una interfaz con los solvers para la resolución de ILP gracias a la librería JOM [Jom]. Por otro lado, el contexto común a ambos estudios comprende el modelado del espacio flex-grid/MCF. Para tales propósitos, se ha considerado que los enlaces de las topologías elegidas son MCF con $C=7,12,19$ número de núcleos por fibra, la parte de red elástica viene modelada por un ancho espectral de 120 FSs de 12.5 GHz cada uno para cada core con tamaño del canal de transmisión variable.

Se asumen demandas de tráfico IP, representadas en una matriz cuadrada proporcional a la población, donde cada elemento de la matriz proporcional al producto de la población de ambos nodos. Para cada demanda y transpondedor se han calculado los $k=5$ caminos más cortos. Las demandas de tráfico son satisfechas gracias a un conjunto de transponders disponibles $(T)$ con line rates $R=40,100,400 \mathrm{Gbps}$, utilizando diferentes formatos de modulación (BSPK,QPSK,16-QAM,64-QAM). Tal información referente a los transponders para diferente $C$, alcance de transmisión óptico (Transmission Reach, TR) y el espacio espectral en FSs utilizado (entre paréntesis), se resume en la tabla 4.4.

Tabla 4.4: Alcance óptico (en Km) y espacio espectral ocupado (en FSs) para el conjunto de transponders, según modulación, line rate y número de núcleos en la fibra.

\begin{tabular}{|r|l|ll|l|l|}
\hline \multirow{2}{*}{$\mathbf{C}$} & \multicolumn{1}{|c|}{ BSPK } & \multicolumn{2}{|c|}{ QSPK } & 16-QAM & 16-QAM \\
\cline { 2 - 6 } & 40 Gbps & 40 Gbps & 100 Gbps & 100 Gbps & 400 Gbps \\
\hline $\mathbf{3}$ & $20000(3)$ & $9000(2)$ & $9000(3)$ & $2000(2)$ & $600(4)$ \\
$\mathbf{1 2}$ & $20000(3)$ & $9000(2)$ & $9000(3)$ & $2000(2)$ & $600(4)$ \\
$\mathbf{1 9}$ & $4755(3)$ & $2383(2)$ & $2383(3)$ & $599(2)$ & $150(4)$ \\
\hline
\end{tabular}

Los valores de alcance óptico de la tabla anterior están influenciados por la interferencia entre los núcleos (ICXT) y es otro de los parámetros para modelar el contexto de fibras multi-core. Tales valores vienen estimados en el trabajo [Perel16a].

\subsubsection{Validación del heurístico}

El conjunto de simulaciones para evaluar el rendimiento de los algoritmos ILP y heurístico para los Flex-grid/MCF-ROADMs FNB y CCC fue ejecutado en un portátil personal con una CPU Intel i7 con 8 núcleos, 16 GB de RAM y 240 GB de SSD. El solver utilizado ha sido IBM CPLEX [Ibm] para la ejecución del ILP y configurado de tal forma que, si tras una hora de ejecución no se encontraba el óptimo, se aportaba la mejor solución. Este último caso solo ha ocurrido muy puntualmente para CCC debido a su aumento de complejidad. Las simulaciones han sido ejecutadas en dos topologías de referencia, 
disponibles en [Net2P], Internet 2 (I2), con un grado nodal promedio de 2.29 y un diámetro de red de $920 \mathrm{~km}$ y Top 7 Spain (T7S), con un grado nodal promedio de 2.89 mientras que el diámetro de red es de $4116 \mathrm{~km}$.

Las figuras 4.8, 4.9, 4.10 y 4.11 exponen los resultados obtenidos por el conjunto de simulaciones anteriormente descrito, bajo el foco del análisis de rendimiento en términos de utilización total del espectro disponible (total de FSs ocupados en la red) frente a la carga total de tráfico soportada por dicha red (throughput) tras recibir la primera demanda que no es posible satisfacer mediante el algoritmo dado. Las tablas 4.5 y 4.6 complementan a las figuras mostrando el throughput total para FNB-ROADMs (referencia) en T7S y I2, respectivamente, en las configuraciones óptimas de fibras y algoritmos, mientras que, para el resto de casos se muestra su diferencia porcentual en rendimiento respecto de su equivalente óptimo.

En primer lugar, tanto en las figuras 4.8, 4.9, 4.10 y 4.11 como en las tablas 4.6 y 4.5 , la validación del algoritmo heurístico frente al ILP puede catalogarse como satisfactoria si realizamos un análisis de rendimiento respecto de costes operacionales (tiempo de ejecución). Los resultados en rendimiento de la cantidad de tráfico que ha podido cursarse gracias al heurístico han sido entre un $10.7 \%$ y un $23.3 \%$ menor que su equivalente en el óptimo aportado por el ILP. Este rango de pérdidas en rendimiento por parte del heurístico puede ser asumible en casos en que la ejecución del ILP pueda ser inabordable, especialmente en grandes redes, debido a su complejidad intrínseca. Además, también proporciona ventaja operacional gracias a un considerablemente menor tiempo de ejecución, de unos pocos segundos, mientras que en el peor de los casos el ILP podría llegar hasta mas de una hora en aportar la solución óptima o incluso no encontrarse.

Por otra parte, en el conjunto de gráficas presentadas en esta subsección, se puede apreciar que las curvas para $C=7$ y 12 siguen la misma tendencia en todos los casos, mientras que, para fibras con 19 cores, la utilización espectral no es tan eficiente. Este hecho se justifica gracias a que para las fibras MCF de 7 y 12 núcleos presentan la misma figura de interferencia ICXT mientras que para 19 cores el ICXT es mucho mas significativo [Perel16a], lo que implica una reducción considerable en el alcance óptico, como se menciona en la tabla 4.4. El aumento en el alcance óptico de transmisión de las demandas reduce el espacio de soluciones aportadas por los transponders disponibles a únicamente los menos eficientes, espectralmente hablando.

Los resultados ponen de manifiesto que no existe una gran diferencia en rendimiento entre utilizar nodos ópticos con total flexibilidad o con restricciones de núcleo. De hecho, las diferencias son tan mínimas que en ningún caso superan el $0.5 \%$ de pérdidas de tráfico en CCC respecto de su configuración homóloga en $\mathrm{FNB}$, tanto en el análisis realizado en la topología Internet 2 como en Top 7 Spain. Respecto a la comparación de rendimiento entre las topologías, ofrece mejor comportamiento, en general, la utilización de fibras MCF en la topología T7S frente a I2. El hecho de tener T7S un diámetro aproximadamente 4 veces menor que $\mathrm{I} 2$ es un elemento diferencial a la hora de la utilización espectral, ya que permite poder utilizar transmisiones ópticas con modulaciones mas eficientes en el espectro. Un análisis mas exhaustivo de las distintas topologías se presenta en la siguiente subseccción. 


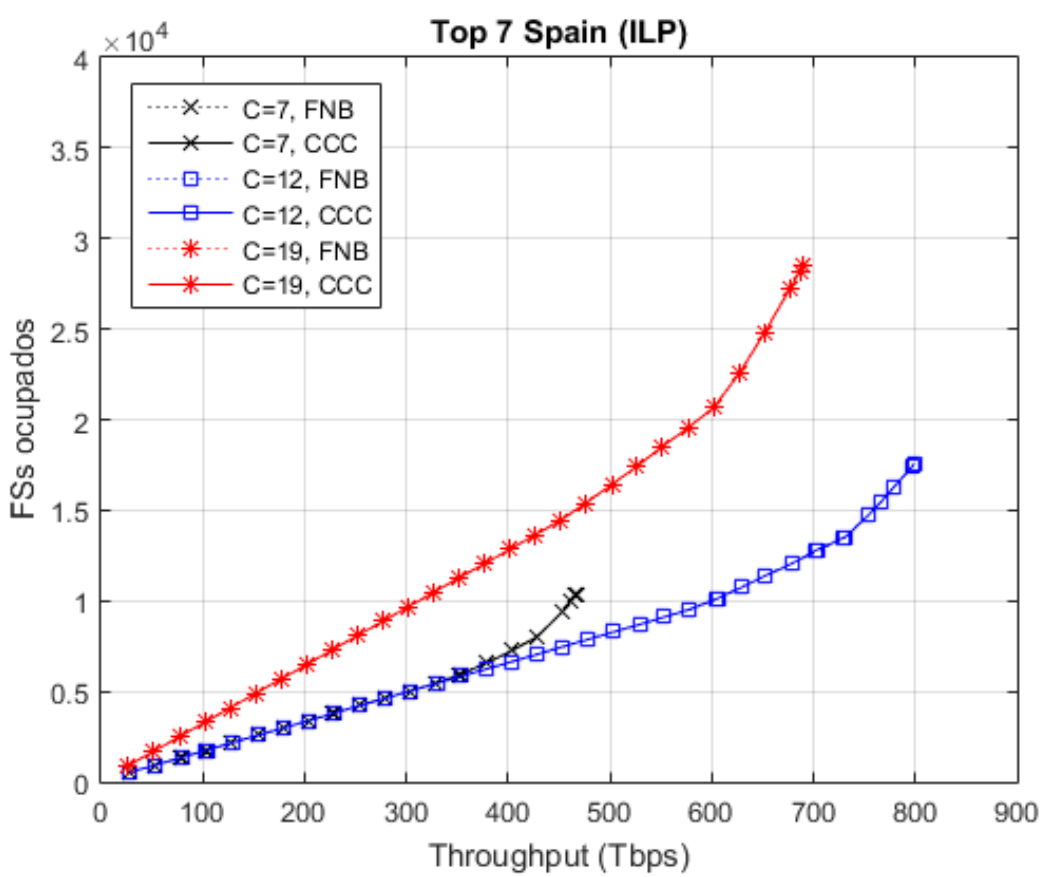

Figura 4.8: Máximo tráfico cursado vs. FS ocupados para ILP en T7S

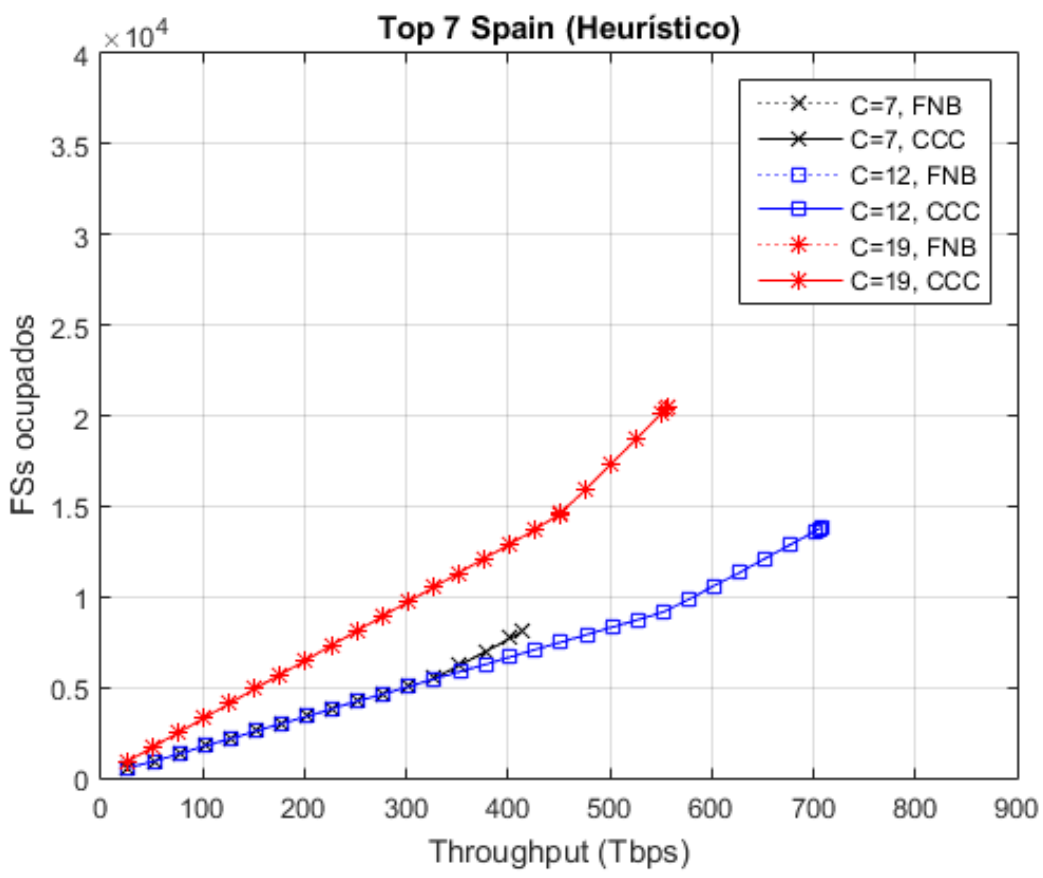

Figura 4.9: Máximo tráfico cursado vs. FS ocupados para heurístico en T7S 


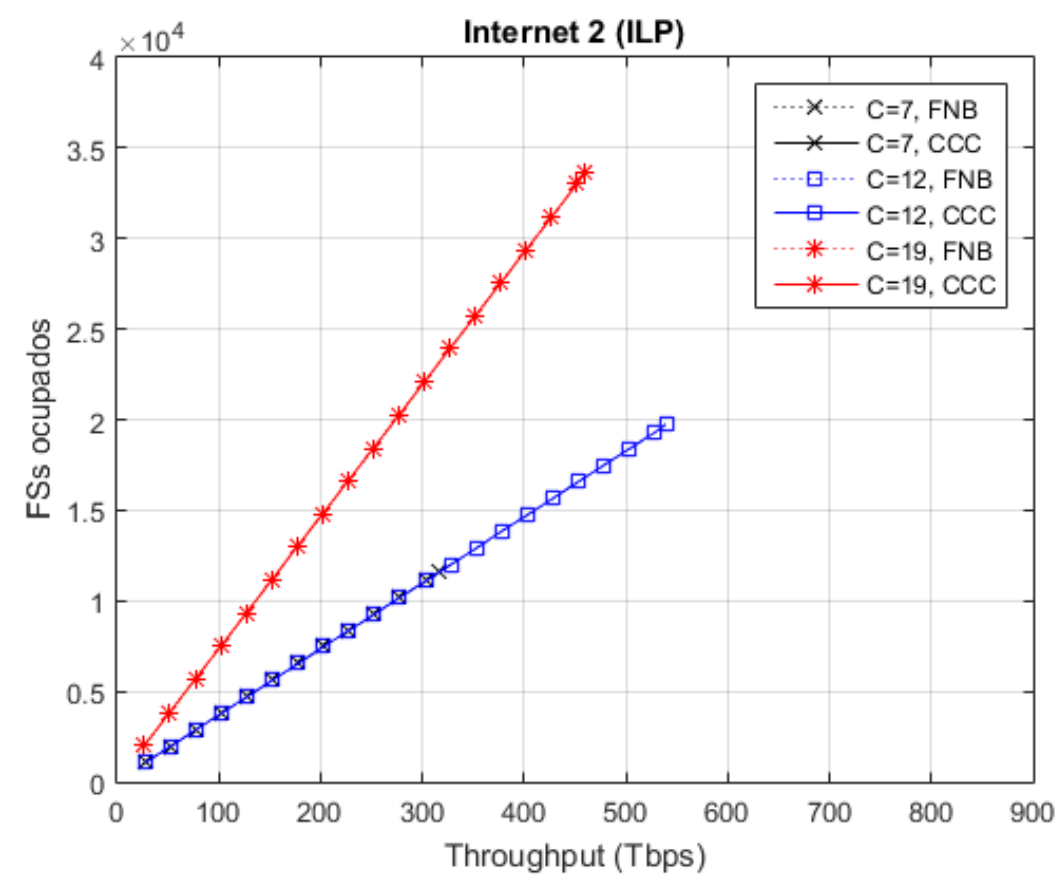

Figura 4.10: Máximo tráfico cursado vs. FS ocupados para ILP en I2

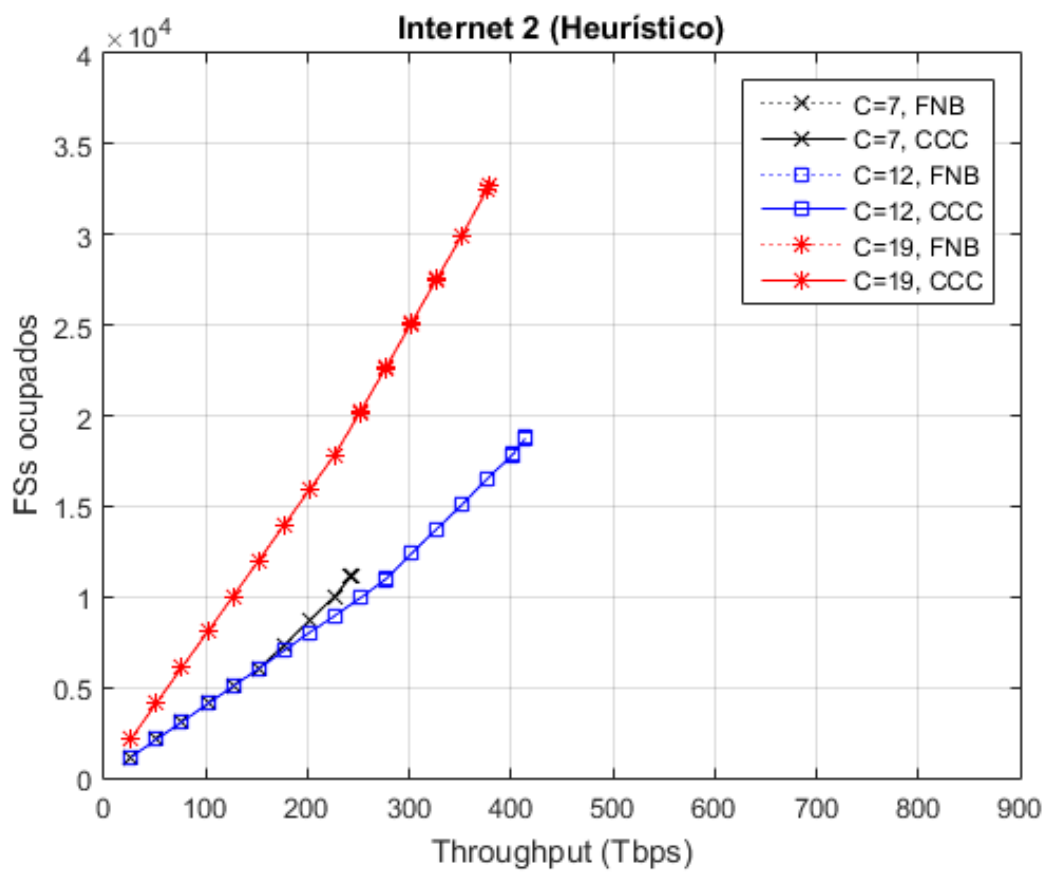

Figura 4.11: Máximo tráfico cursado vs. FS ocupados para heurístico en I2 
Tabla 4.5: Throughput total en T7S usando ILP frente al heurístico

\begin{tabular}{l|ll|ll|ll}
\hline & \multicolumn{2}{|c|}{ C=7 } & \multicolumn{2}{c|}{ C= 12 } & \multicolumn{2}{c}{ C= 19 } \\
& FNB & CCC & FNB & CCC & FNB & CCC \\
\hline ILP & $\mathbf{4 6 4 , 0 0}$ & $0,00 \%$ & $\mathbf{7 9 8 , 7 0}$ & $-0,14 \%$ & $\mathbf{6 8 9 , 7 0}$ & $-0,48 \%$ \\
Heurístico & $-10,73 \%$ & $-10,73 \%$ & $-11,46 \%$ & $-11,64 \%$ & $-19,25 \%$ & $-19,53 \%$ \\
\hline
\end{tabular}

Tabla 4.6: Throughput total en I2 usando ILP frente al heurístico

\begin{tabular}{l|ll|ll|ll}
\hline & \multicolumn{2}{|c|}{ C=7 } & \multicolumn{2}{c|}{ C= 12 } & \multicolumn{2}{c}{ C= 19 } \\
& FNB & CCC & FNB & CCC & FNB & CCC \\
\hline ILP & $\mathbf{3 1 5 , 6 2}$ & $-0,09 \%$ & $\mathbf{5 3 9 , 6 2}$ & $-0,11 \%$ & $\mathbf{4 6 0 , 6 4}$ & $-0,20 \%$ \\
Heurístico & $-23,14 \%$ & $-23,14 \%$ & $-23,31 \%$ & $-23,31 \%$ & $-17,82 \%$ & $-18,23 \%$ \\
\hline
\end{tabular}

\subsubsection{Análisis de rendimiento de CCC-ROADM}

De forma similar al estudio anterior, las simulaciones se han llevado a cabo en un portátil personal con una CPU i7 con 8 núcleos lógicos y 16 GB de memoria. Este estudio pretende analizar el rendimiento de la idea CCC en los nodos ópticos frente a la opción mas flexible (FNB) resolviendo el problema RMSCA gracias al algoritmo heurístico, para cuatro topologías de referencia, Poland (POL), Germany 50 (GER), NSFNet (NFS) y la red óptica europea (EON), todas disponibles en [Sdnli17]. Las principales características de las topologías seleccionadas se exponen en la tabla 4.7. Nótese que se tienen en cuenta dos topologías con un diámetro inferior a $1000 \mathrm{~km}$, consideradas como redes pequeñas, mientras que las otras dos redes restantes tienen un tamaño cercano al alcance óptico máximo considerado de una fibra MCF con 19 cores.

Tabla 4.7: Métricas relativas a las redes de referencia

\begin{tabular}{c|cccc}
\hline Topología & Nodos & Enlaces & Grado nodal & Diámtro (km) \\
\hline POL & 12 & 36 & 3.00 & 810 \\
GER & 50 & 176 & 3.52 & 934 \\
NFS & 14 & 42 & 3.00 & 4500 \\
EON & 18 & 66 & 3.67 & 3837 \\
\hline
\end{tabular}

El modelo de tráfico consiste en una distribución aleatoria uniforme entre 0 y 1 para cada par de nodos de la topología. El resto de consideraciones se asumen compartidas con el estudio presentado en la subsección 4.4.2.1. El máximo tráfico llevado por la redes para flex-grid/MCF ROADM FNB y CCC para las cuatro topologías evaluadas y tres valores de $C$ se presenta en el gráfico de barras representado por la Figura 4.12 .

Como era de esperar, para todo $C$ analizado, las redes de pequeño diámetro se ven beneficiadas por el amplio número de posibles transponders para poder satisfacer las demandas de tráfico cumpliendo con las exigencias de alcance óptico, utilizando modulaciones 

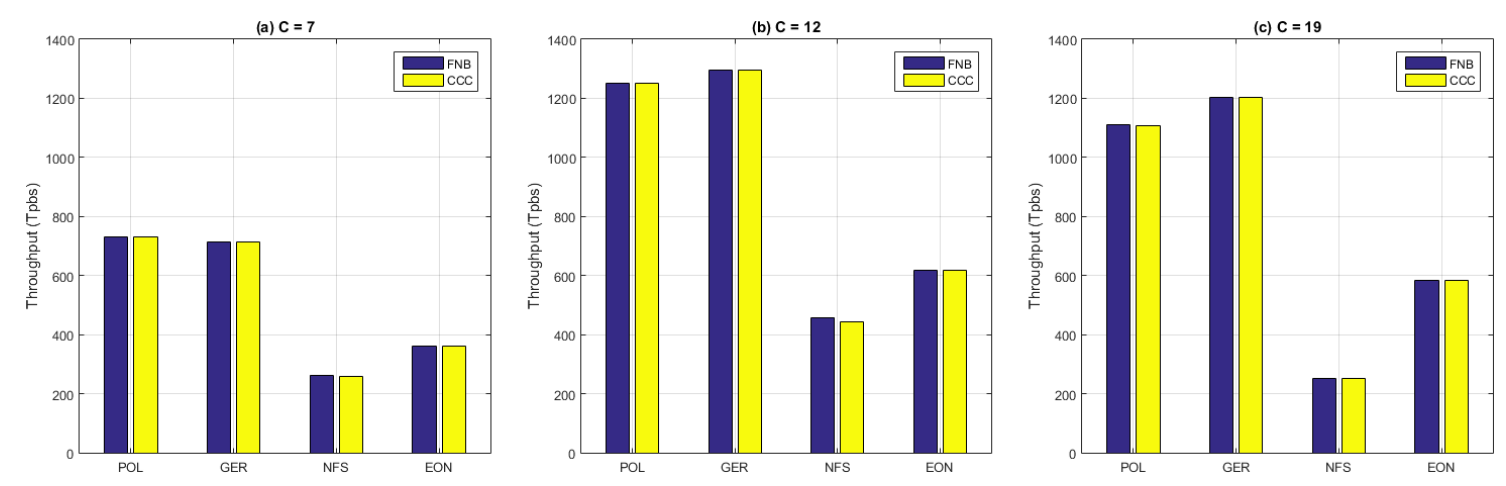

Figura 4.12: Máximo tráfico llevado según el heurístico para diferentes topologías y número de núcleos: (a) 7, (b) 12 y (c) 19.

eficientes desde un punto de vista espectral. Sin embargo, las dos topologías mas amplias, cuyo diámetro impide el uso de modulaciones eficientes, especialmente para $C=19$, presentan mayores problemas para resolver el RMSCA, ya que, el espacio de soluciones es menor. Además, los resultados parecen apuntar a un beneficio extra para aprovechar los recursos de la red en aquellas con un alto grado de enlaces, favoreciendo encontrar caminos para satisfacer las demandas de tráfico, especialmente con un alto nivel de carga. Las redes que se alejan de una topología tipo malla, son susceptibles de tener efecto de cuello de botella en alguno de sus enlaces, creando aislamiento en de nodos distantes entre sí para entornos con cargas altas de tráfico. La topología de este tipo es NFSNet, esta razón unida a su alto valor de diámetro de red, explica que sea la opción con peor rendimiento.

Respecto a la comparación en rendimiento de CCC frente a FNB, como ya anticipaba el estudio anterior, las diferencias se presentan como muy menores. De nuevo, tales resultados afianzan la idea de continuidad de núcleo como buena opción para ser utilizada en nodos ópticos según el balance rendimiento-coste para un ROADM bajo contextos de redes elásticas $\mathrm{MCF}$.

Con el fin de cuantificar el rendimiento del algoritmo heurístico aplicado a distintas topologías y número de cores en las fibras, se define eficiencia económica de la red como la relación entre el máximo throughput de la red (beneficio) y el número total de cores y enlaces de dicha red (coste). La tabla 4.8 resume los resultados obtenidos para todas las redes y fibras consideradas.

Tabla 4.8: Eficiencia económica de la red para 4 topologías diferentes

\begin{tabular}{lccc}
\hline Topología & $\boldsymbol{C}=7$ & $\boldsymbol{C = 1 2}$ & $\boldsymbol{C = 1 9}$ \\
\hline POL & 2,9 & 2,9 & 1,6 \\
GER & 0,6 & 0,6 & 0,4 \\
NFS & 0,9 & 0,9 & 0,3 \\
EON & 0,8 & 0,8 & 0,5 \\
\hline
\end{tabular}

En dicha tabla se pueden apreciar las implicaciones de utilizar fibras con un alto valor de ICXT (asociado a una reducción de alcance de transmisión óptica), para todas las topologías el uso de fibras con 19 cores reduce considerablemente la métrica de eficiencia 
económica. Por otro lado, el análisis entre topologías revela una ventaja económica en utilizar fibras MCF, especialmente para 7 y 12 núcleos, para redes de bajo diámetro y que no necesiten una gran inversión en fibra (número de enlaces), mientras que para contextos con una gran número de fibras y/o tamaño el beneficio relativo no está asegurado.

\subsection{Análisis de escenario dinámico}

Esta parte del trabajo presenta una evaluación de las arquitecturas que se detallan en la sección 4.3.4.2 dentro de un contexto de redes elásticas con fibras basadas en SDM en un escenario dinámico. Nótese que este estudio se diferencia en esencia del propuesto en la sección anterior en dos principales puntos. En este caso, se extiende el escenario asumido anteriormente para fibras multi core a un contexto general del concepto SDM. Por otro lado, la problemática a resolver en el escenario dinámico va orientado al aprovisionamiento de demandas de tráfico a lo largo de un tiempo de simulación en lugar de una planificación de red bajo requerimientos de tráfico conocidos previamente. Los resultados y discusión de este estudio han sido publicados en la revista Computer Communcations (véase [Rumip18]). El desarrollo y evaluación de este estudio ha formado parte del trabajo de la presente tesis.

\subsubsection{Algoritmo heurístico RMSSA}

El algoritmo desarrollado para resolver el problema de asignación de modulación, espectro y canal espacial (RMSSA) en entornos de redes flexibles, dinámicos (On-line) y con consideraciones de súper canales (Spa-SCh y Spe-SCh) trata de un proceso heurístico basado en la técnica greedy [Pavon16]. El pseudocódigo del algoritmo viene representado en la próxima Figura 4.13 .

El algoritmo se nutre con el conjunto de entradas compuesto por la topología, las bandas de guarda para el Spe-SCh $(G B)$, una demanda a satisfacer $(d)$ y el número de sub-canales para formar el SCh $\left(n_{s}\right)$. El algoritmo comienza computando los $K=3$ caminos candidatos más cortos para satisfacer cada demanda de tráfico y se almacenan en un conjunto de caminos candidatos. La línea 5 del pseudocódigo marca el inicio del bucle principal del algoritmo donde se analizan de forma iterativa todos los caminos candidatos $(p \in \mathcal{P})$. En primer lugar, se selecciona la modulación mas eficiente, siempre que su alcance óptico asociado sea mayor que la distancia recorrida $p$. A continuación, es necesario calcular el número de FS $\left(n_{f s}\right)$ para satisfacer la demanda según la siguiente expresión:

$$
n_{f s}=\frac{\frac{r_{d}}{n_{s} \cdot S E}+G B}{\Delta_{f s}}
$$

con $r_{d}$ siendo el bit-rate de la demanda $d, S E$ la eficiencia espectral de la modulación seleccionada y $\Delta_{f s}$ el ancho espectral de un FS unitario. En caso de utilizar súper canales espectrales, $n_{s}=1$, mientras que el número de Spa-SChs viene determinado por el mínimo que satisfaga el cantidad mínima de FSs necesaria para alojar el line-rate de la demanda [Rumip17b]. Las líneas 10 a 22 analizan, para cada espacio $s_{i}$ de la fibra, el cumplimiento de las exigencias de continuidad y contigüidad espectral a lo largo del todo el camino 


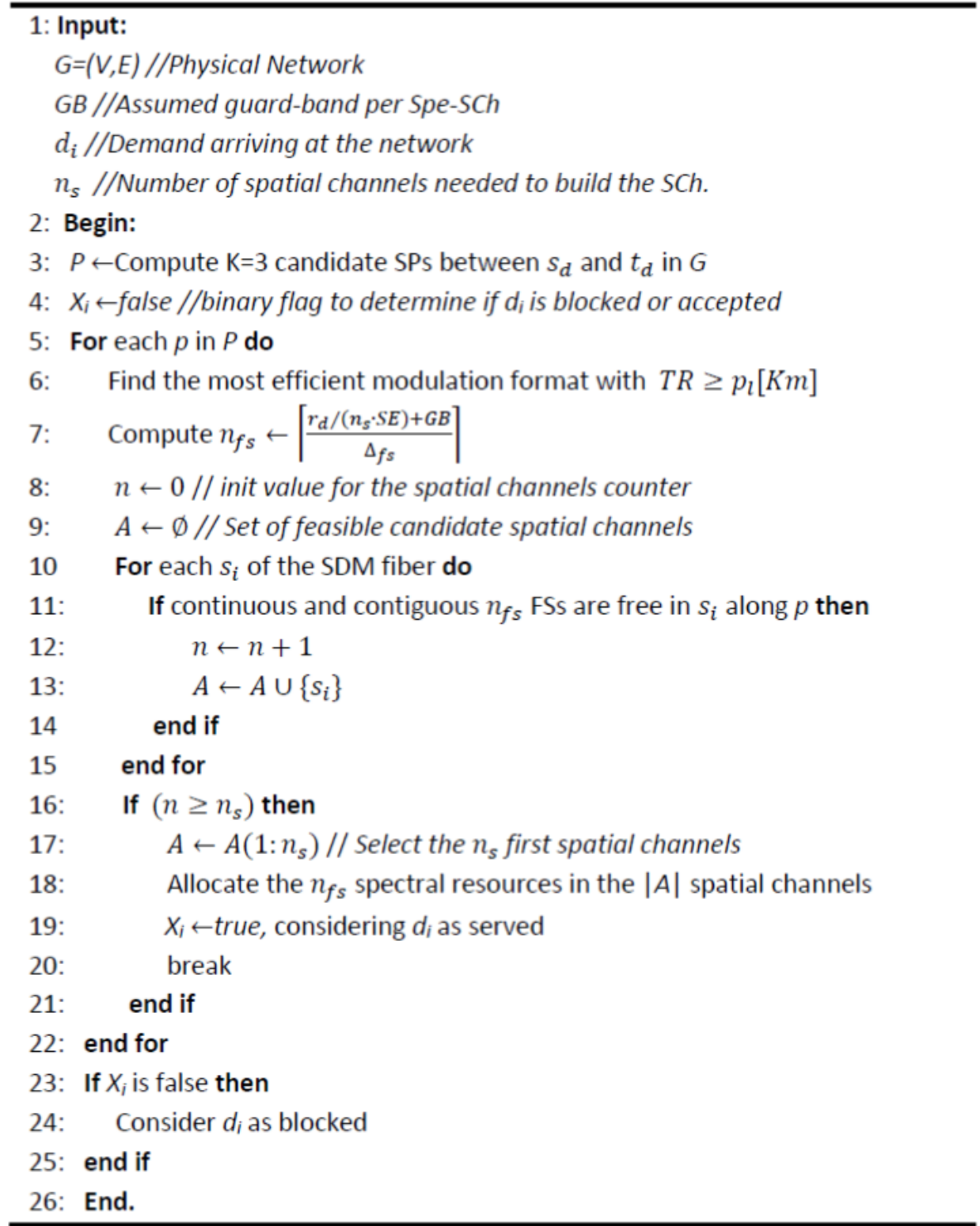

Figura 4.13: Pseudocódigo del algortimo heurístico para resolver el RMSSA. Fuente: [Rumip18]

$p$ características de las redes elásticas, así como core si fuera necesario. La asignación espectral se asume tipo first-fit. En caso de que se cumplan los requerimientos previos, la demanda se asume como satisfecha, en caso contrario se consideran nuevos candidatos hasta que no haya mas disponibles, en tal situación la demanda de tráfico es rechazada.

\subsubsection{Configuración del escenario dinámico}

El contexto de simulación que se ha considerado para realizar un estudio de evaluación de throughput, según las propuestas de SDM-ROADMs de la sección 4.3 y bajo un 
escenario dinámico, se expone en esta subsección. El objetivo principal es el de comparar en términos de rendimiento las arquitecturas R\&S SCC SDM-ROADM frente a las referencias de ROADMs, JoS y FNB, teniendo en cuenta consideraciones de súper canales espaciales y espectrales. El escenario dinámico asume demandas de tráfico según una distribución de Poisson centrada en tiempos promedio de entrada (inter-arrival time) y establecimiento (holding time). Se han tenido en cuenta dos perfiles de tráfico diferentes para las demandas, TP1 $=100,400,1000 \mathrm{Gbps}$ y bit-rate y TP2 $=400,1000,2000 \mathrm{Gpbs}$ con una probabilidad para ambos perfiles de $\mathrm{P}_{T}=0.4,0.3,0.3$ respectivamente.

Las topologías elegidas para albergar el estudio han sido: una red continental, la red óptica europea de 16 nodos (EON16) con 23 enlaces y un diámetro de red de $2693 \mathrm{~km}$ y una red de menor tamaño, Deustche Telekom Optical Network, DT12, con 12 nodos, 20 enlaces y 1019 km de diámetro de red. Los enlaces se consideran SDM, con multiplicidades espaciales $S=[7,30]$, incluyendo los casos de fibras multi-núcleo que presenten prototipos para tal valor de $S$ [SSakag12] [Sakag14][Puttn17]. Las estimaciones de ICXT en las fibras MCF utilizadas son las aportadas en [Poggi14] y han propiciado la base para asignar los valores de transmisión óptica a las fibras SDM, utilizando diversos formatos de modulación (tabla 4.9). Los valores resaltados en negrita hacen referencia a las fibras afectadas por el ICXT.

Tabla 4.9: Estimación de alcance óptico en km en fibras SDM

\begin{tabular}{|c|c|c|c|c|}
\hline $\boldsymbol{S}$ & BPSK & QPSK & 16-QAM & 64-QAM \\
\hline $\mathbf{7}$ & $>20000$ & 9000 & 2000 & 600 \\
\hline $\mathbf{1 2}$ & $>20000$ & 9000 & 2000 & 6000 \\
\hline $\mathbf{1 9}$ & $\mathbf{4 7 5 5}$ & $\mathbf{2 3 8 3}$ & $\mathbf{5 9 9}$ & $\mathbf{1 5 0}$ \\
\hline $\mathbf{2 2}$ & $\mathbf{6 6 0 7}$ & $\mathbf{3 3 1 1}$ & $\mathbf{8 3 2}$ & $\mathbf{2 0 9}$ \\
\hline $\mathbf{3 0}$ & $\mathbf{1 5 8 4 9}$ & $\mathbf{7 9 4 3}$ & $\mathbf{1 9 9 5}$ & $\mathbf{5 0 1}$ \\
\hline
\end{tabular}

Cada fibra flex-grid/SDM se considera con disponibilidad espectral de 320 FS de un ancho $\Delta_{f s}=12.5 \mathrm{GHz}$ y la banda de guarda $G B=7,5 \mathrm{GHz}$. La evolución de la carga de tráfico ofrecido en la red se ha modelado fijando el tiempo promedio de llegada y aumentando progresivamente el tiempo de establecimiento hasta obtener una probabilidad de bloqueo entorno al $1 \%$. El volumen de demandas de tráfico por cada simulación ha sido de $5 \cdot 10^{5}$.

\subsubsection{Resultados y discusión}

Las figuras 4.14 y 4.15 exponen el conjunto de ejecuciones llevadas a cabo para realizar el estudio de rendimiento en throughput en función del número de multiplicidades espaciales en las fibras SDM y para distintos diseños R\&S flex-grid/SDM-ROADMs. Las líneas continuas hacen referencia al comportamiento observado por las fibras tipo MF, mientras que los puntos aislados hacen referencia a los obtenidos para los valores existen de fibras MCF.

La Figura 4.14 muestra una comparativa entre el uso de SCC o FNB SDM-ROADMs y Spe-SCh en términos de throughput, es decir, cuánto tráfico total puede ser llevado por 
una red que utilice tales diseños de ROADMs y súper canales espectrales. Como ya anticipaba el análisis estático (MCF), las diferencias en throughput de SCC frente a FNB, son ciertamente pequeñas, menores al $2 \%$ en una red de tamaño nacional como DT12. Sin embargo, para la red continental EON16 las diferencias entre ambos diseños ascienden a un $14 \%$ mayor en rendimiento a favor del diseño FNB, marcado como benchmark. Al aumentar el diámetro de la red, algunas de las modulaciones, espectralmente mas eficientes, no consiguen alcanzar los especificaciones de longitud del camino, siendo necesario la utilización de modulaciones que necesitan mayor espectro para poder servir el tráfico, este hecho es mas determinante cuando existen restricciones de canal espacial (SCC) o perfil de tráfico alto. Esta última limitación se puede apreciar en mayor medida en la red DT12, donde para 2.3 veces más trafico ofrecido promedio en TP2 respecto de TP1, únicamente puede llevar un $14 \%$ mas de tráfico.
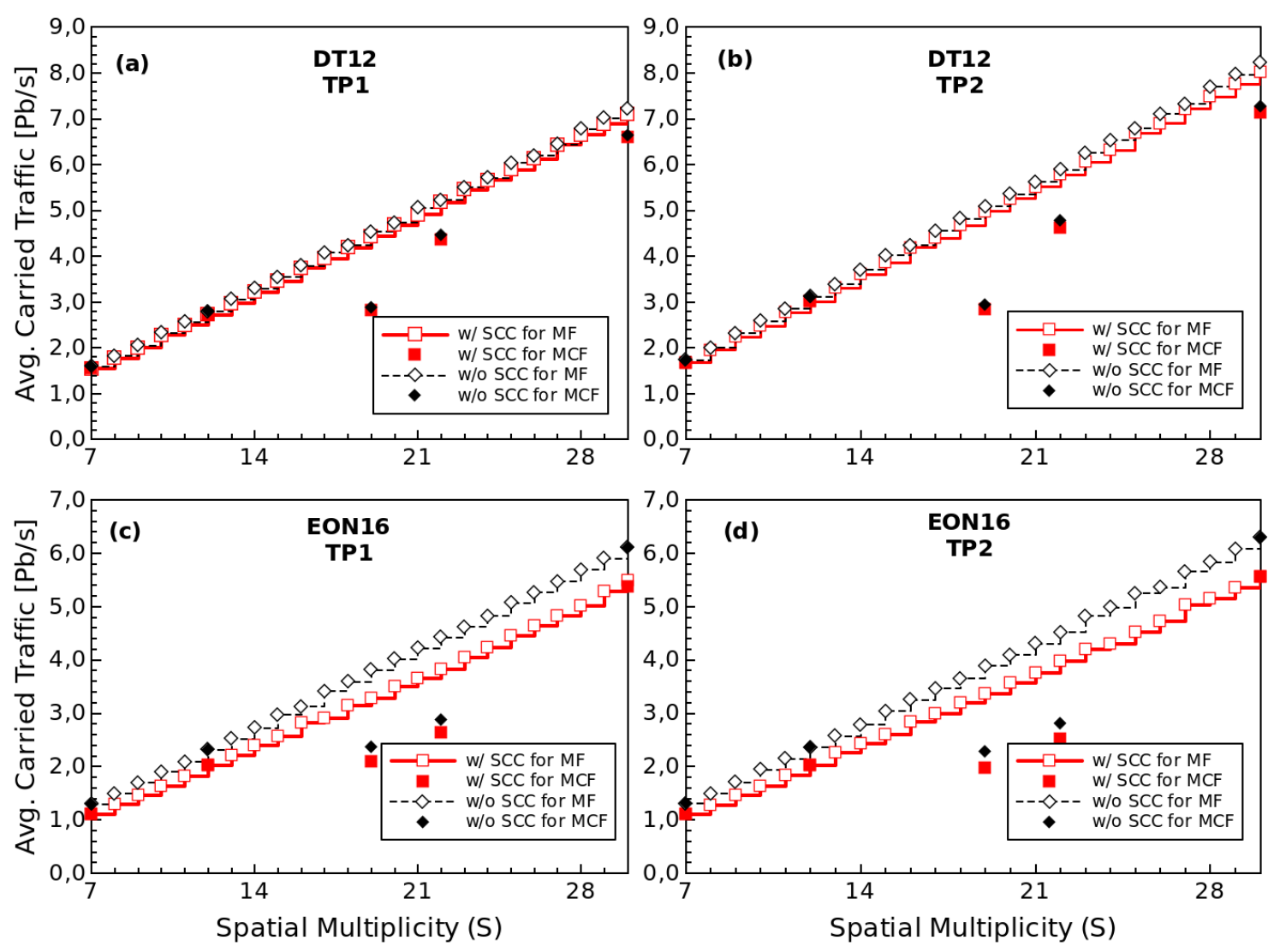

Figura 4.14: Tráfico total en la red (en Pbps) frente a multiplicidad espacial $(S)$ con SpeSCh en: (a) DT12 con TP1, (b) DT12 con TP2, (c) EON16 con TP1 y (d) EON16 con TP2. Fuente: [Rumip18].

$\mathrm{El}$ análisis de MF frente a MCF revela que para los prototipos considerados de fibras multi-core con $C$ igual a 7, 12 y 30, el bajo ICXT ha favorecido que el rendimiento sea prácticamente igual que en MF. Por otro lado, para los prototipos de fibras con 19 y 22 núcleos, donde el alcance de transmisión óptica es menor (véase tabla 4.9), existe una evidente degradación en la cantidad de tráfico total que se puede llevar en la red. Un hecho especialmente notorio es que, tanto para DT12 como EON16, utilizar fibras de 
19 cores/espacios conlleva incluso peor rendimiento que el uso de fibras con $S=12$ (o núcleos).

La Figura 4.15 expone los resultados obtenidos tras la simulaciones orientadas a comparar el rendimiento en throughput de la propuesta SCC SDM-ROADM cuando se utilizan Spa-SCh frente a las referencias JoS y FNB. La primera diferencia clara en el uso de SpeSCh o Spa-SCh es que para éste último caso, independientemente del tipo de ROADM a considerar, el aumento de $S$ no implica necesariamente mayor rendimiento, pudiendo incluso llegar a una disminución. La restricción de espacio espectral en la asignación de súper canales espaciales es una de las causas de dicho fenómeno. Supóngase, que se desea dar cabida a una demanda de $r_{d}=400 \mathrm{Gbps}$, con $S=7, G B=7.5 \mathrm{GHz}$ y modulación 16QAM, el número de FS es 2, mientras que el mismo contexto, salvo con $S=8$, resultaría igualmente 2. Además, puede ocurrir que el comportamiento avaro de la asignación de Spa-SChs implique una infrautilización de los recursos espectrales que impida establecer nuevos lightpaths, siendo en tal caso preferible un valor de $S$ menor.
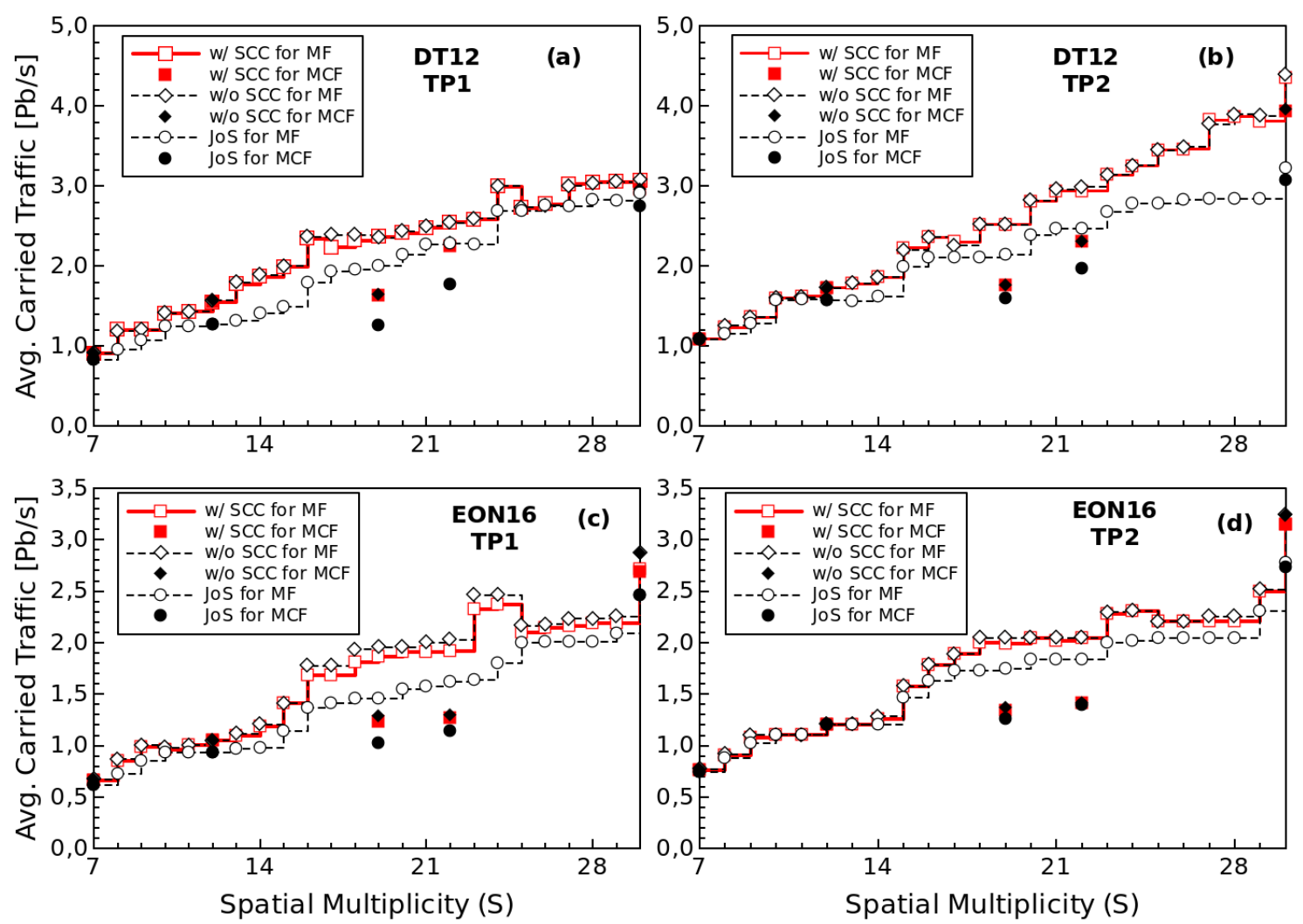

Figura 4.15: Tráfico total en la red (en Pbps) frente a multiplicidad espacial $(S)$ con SpaSCh en: (a) DT12 con TP1, (b) DT12 con TP2, (c) EON16 con TP1 y (d) EON16 con TP2. Fuente: [Rumip18].

De nuevo también para Spa-SCHs, el rendimiento del diseño SCC ha sido muy elevado comparado con $\mathrm{FNB}$, menor al $1 \%$ tanto para escenarios $\mathrm{MF}$ como $\mathrm{MCF}$, mientras que ha sido claramente mayor a JoS, siendo en este caso hasta un $40 \%$ peor que para la opción FNB. La comparativa del caso MCF revela nuevamente la pérdida de rendimiento para 19 y 22 núcleos debido a su alto valor en ICXT, siendo el peor escenario para 19 cores donde 
se deja de llevar hasta un $58 \%$ de tráfico respecto de su homólogo en MF. La sensibilidad a la figura de tráfico queda de nuevo en evidencia en el caso de asignación de Spa-SChs, por ejemplo, para la red EON16 y $S=30$ se obtiene un aumento de throughput del $16 \%$ para TP1 mientras que para TP2 se consigue obtener un incremento de tráfico total del $42 \%$.

Finalmente, tanto para los resultados obtenidos, tanto en la Figura 4.14 como 4.15, las diferencias entre FNB SDM-ROADM y SCC son prácticamente iguales en las soluciones MCF y MF. Como ejemplo, la Figura 4.14(d), en la red EON16 y una multiplicidad espacial de $S=19$, las diferencias son cercanas al $15 \%$ (3.9 Pbps y 3.4 en MF y 2.3 Pbps y 2 para $\mathrm{MCF}$ ). Hecho que pone de manifiesto que la única dependencia evidente en este caso es la penalización por ICXT.

\subsubsection{Estudio tecno-económico}

Con el fin de contextualizar los diseños para SDM-ROADMs dentro de un marco de coste económico y poder realizar una evaluación del balance rendimiento-coste, se ha propuesto un breve análisis centrado en la economía del hardware de los dispositivos mas relevantes en el ROADM, los SSSs. La tabla 4.10, presentada en [Rivas17], expone un modelo de coste normalizado para los dispositivos SSS en función de su tamaño (número de entradas/salidas) donde se aprecia el aumento del coste conforme crece el número de puertos en el dispositivo.

Tabla 4.10: Coste normalizado de por SSS. Fuente: [Rivas17]

\begin{tabular}{|c|c|}
\hline Tamaño SSS & Coste Normalizado \\
\hline $1 \times 5$ & 0.63 \\
\hline $1 \times 9$ & 1 \\
\hline $1 \times 20$ & 1.58 \\
\hline $1 \times 40$ & 2.50 \\
\hline $1 \times 80$ & 3.95 \\
\hline $1 \times 160$ & 6.25 \\
\hline
\end{tabular}

Esta tabla, conjuntamente analizada con la tabla 4.3 , coste por nodo, ha dado como resultado el llamado coste de red, siendo tal una suma total de los coste totales por nodo. Estos resultados son resumidos en la tabla 4.11 para las dos topologías de referencia y tres valores de $S$. En ella se puede apreciar claramente que la opción mas cara es sin duda FNB que se presenta como entre 3 y 6 veces con mayor coste que su equivalente en SCC. Por otro lado, la opción JoS emerge como el diseño que menos coste de red asociado al uso de súper canales espaciales, reduciendo su coste hasta la mitad en algunos casos respecto de la arquitectura SCC. 
Tabla 4.11: Coste de red según tamaño para distintos SDM-ROADM.

\begin{tabular}{|c|c|c|}
\hline \multirow{2}{*}{ ROADM } & \multicolumn{2}{|c|}{ Coste para $\boldsymbol{S}=\mathbf{7 / 1 9 / 3 0}$} \\
\cline { 2 - 3 } & DT12 & EON16 \\
\hline FNB & $301 / 1325 / 322$ & $325 / 1511 / 3771$ \\
\hline SCC & $106 / 287 / 454$ & $141 / 383 / 604$ \\
\hline JoS & $66 / 113 / 150$ & $71 / 140 / 200$ \\
\hline
\end{tabular}

\subsection{Conclusiones}

Este capítulo se centrado en el tema de la expansión de redes bajo el foco de las tecnologías SDM y flex-grid como posibles soluciones para abordar los problemas en redes de transporte asociados al crecimiento del tráfico IP. Se ha propuesto una idea principal donde se restringe el canal espacial (núcleo, en redes con enlaces MCF) para la satisfacción de demandas de tráfico. Para ello, se define un diseño que implementa la idea de restricción espacial/core y se presenta una comparación con la referencia libre de restricciones a nivel de complejidad.

Los resultados han presentado dicha propuesta como una solución válida y relativamente poco compleja a utilizar para fines SDM, ya que puede reusar diseños de nodos ópticos existentes en redes SMF. Por otra parte, se ha probado la validez de la propuesta de ROADM, SCC, dentro de dos marcos diferentes, escenario estático (off-line) y escenario dinámico (on-line). Los resultados de ambos análisis han mostrado que el rendimiento de la propuesta SCC ha sido muy similar al mostrado por la opción FNB, especialmente cercanos (menor al $1 \%$ en el peor de los casos) en el contexto de planificación de red. Este comportamiento, conjuntamente analizado con el beneficio a nivel de coste y complejidad, puede concluir que para un gran numero de escenarios de la planificación o expansión de las redes, la restricción de núcleo (o espacio) se muestra como una opción a considerar. 


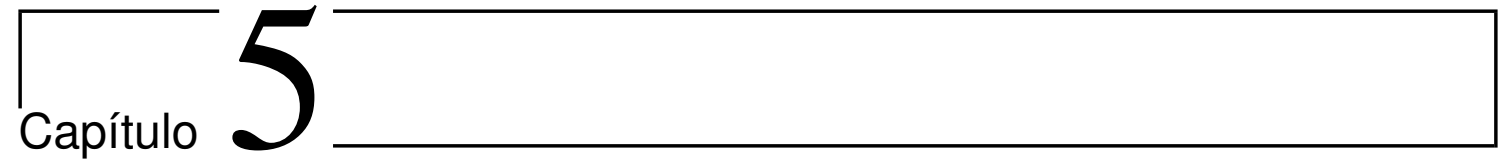

\section{Conclusiones y líneas futuras}

5.1. Conclusiones ......................... 87

5.2. Trabajos futuros $\ldots \ldots \ldots \ldots \ldots \ldots \ldots \ldots$

En este capítulo se resumen las principales aportaciones y conclusiones derivadas de este trabajo y presenta una breve discusión sobre las posibles lineas de trabajo futuras.

\subsection{Conclusiones}

El primer capítulo de este trabajo trata de contextualizar el marco de esta tesis analizando el cambio de paradigma en las redes de telecomunicaciones causado, en parte, por el tráfico basado en la nube, además de analizar las posibles implicaciones en las redes de transporte de las predicciones del crecimiento de tráfico IP y el cumplimiento de los objetivos 5G. Tal entorno ha servido como motivación para la existencia de la tesis, orientada a evaluar soluciones que afronten los anteriormente mencionados desafíos.

Por otra parte, el segundo capítulo presenta una investigación sobre el posible impacto real de las predicciones para los próximos años en las redes de transporte desde el punto de vista del crecimiento y expansión de las grandes redes de distribución de contenido (CDNs) y asumiendo un modelado de tráfico IP sensible a los tipos principales de servicios disponibles en la actualidad. Los resultados de dichos estudios presentan un panorama menos agresivo que el dado por las previsiones de [Cisco16a] o [Cisco17], donde el acercamiento de contenido al usuario dado por la expansión y proliferación de CDNs puede parcialmente aliviar el impacto en los enlaces de las redes de transporte. Cabe mencionar que los resultados concluyen que el contexto de los próximos años dependerá ineludiblemente de la naturaleza de los servicios asociadas a las aplicaciones futuras, siendo especialmente relevante el balance promedio entre el tráfico a usuario y el tráfico de sincronización de contenido. Se concluye además, a la vista de los resultados, que el crecimiento neto de tráfico en las redes troncales para los próximos años será menor que el anunciado por las previsiones actuales.

Las redes definidas por software y la virtualización de funciones de red han formado 
parte relevante de esta tesis y se presentan en el capítulo tercero. Se ha introducido un análisis sobre el papel de la optimización y la planificación dentro del contexto SDNNFV aportando un conjunto de casos de uso representativos. Alguno de tales casos de uso han servido como motivación para realizar dos pruebas de concepto donde se ha tratado de mostrar el beneficio de herramientas open source en la gestión de redes con capacidades SDN y NFV. Los resultados en este campo arrojan un mensaje claro, la correcta simbiosis entre SDN y NFV será concluyente para el cumplimiento de los objetivos 5G, donde la latencia se considera como una métrica esencial, siendo las redes basadas en SDN-NFV una posible opción para abordar los desafíos del panorama futuro en las redes.

Finalmente, el capítulo cuatro ha atendido la necesidad de un aumento de la capacidad en las redes de transporte como soporte del aumento de tráfico según la tecnología SDM. Este enfoque se ha visto enriquecido con la propuesta de continuidad en el canal espacial a lo largo de todo el camino de un lightpath. Se ha presentado una candidatura (SCC) para una hipotética implementación de dicha idea en un nodo óptico gestionado por el dispositivo ROADM. El análisis de esta propuesta de ROADM, comparada con su referencia totalmente flexible, revela que SCC aporta una considerable menor complejidad especialmente para un alto grado nodal y/o espacial. También se ha estudiado el rendimiento del SCC SDM-ROADM en escenarios de tráfico estático susceptible de planificación previa y un escenario dinámico orientado al aprovisionamiento de tráfico en la red. Se ha observado que la pérdida de rendimiento ha sido muy baja en comparación con su diseño de referencia FNB. Este hecho, sumado a su menor complejidad, hacen de la propuesta SCC SDM-ROADM una opción interesante a implementar en las redes ópticas flex-grid que puedan soportar el crecimiento del tráfico IP en los próximos años.

\subsection{Trabajos futuros}

Siempre con el objetivo de investigar con los desafíos propios del 5G, proyectos de colaboración como Metro-Haul, pueden ayudar a alcanzar tal horizonte a través de nuevas arquitecturas de red basadas en nuevos tipos de nodo, de acceso y metro, con capacidad de almacenamiento y cómputo, que integren los conceptos de desagregación de hardware, virtualización, automatización y programabilidad (control SDN). Este tipo de planteamientos pueden conseguir reducciones en el consumo de energía y en costes CAPEX y OPEX además de expandir el horizonte a nuevos servicios basados en el Internet de las Cosas, Internet táctil e Industria 4.0.

El trabajo derivado de esta tesis doctoral deja abiertos numerosos frentes de investigación en el campo de la gestión y planificación de la redes de telecomunicaciones. En el tiempo de la escritura este trabajo, el doctorando está activamente colaborando en varias ramas de la investigación en comunicaciones ópticas:

- El trabajo [Moren18b] sobre la evolución temporal de las redes core asumiendo el crecimiento de CDNs, presentado en el capítulo 2, se encuentra en proceso de revisión en la revista Elservier Computer Networks.

- Una extensión de Net2plan con integración de información geográfica se ha presentado en el congreso ICTON 2018, conjunto a un caso de uso centrado en la ciudad 
de Cartagena, con vistas a ser un marco de simulación fiable en el contexto de redes de acceso 5G [Romer18]. Este trabajo ha servido como base para el desarrollo de una versión mas completa y extendida a las provincias de Murcia y Alicante con el objetivo de analizar el papel de la latencia en el dimensionamiento de redes basadas en SDN-NFV. Dicho trabajo está en revisión en la revista IEEE/OSA JOCN, en el special issue sobre latencia en Edge Optical Networks [Garri18b].

- Se están realizando avances en el proceso de descubrimiento de red desde la perspectiva de un controlador SDN en sistemas parcialmente desagregados. En el trabajo propuesto para su presentación en el congreso OFC 2019, se muestra una prueba de concepto donde Net2plan recibe los parámetros de configuración de transponders de Nokia con agentes basados en OpenConfig gracias al uso de la NBI establecida entre el controlador ONOS y la capa de aplicación [Garri19a].

- Una extensión de los trabajos [Moren18b] y [Moren18d] se ha propuesto para presentarse como demostración en el congreso OFC 2019. En este trabajo se asume además una red de transporte IP sobre capa óptica emulada y centrada en la gestión multi datacenter y multi tenant [Garri19b]. 



\section{Bibliografía}

[5Gkpi] "5G Infrastructure Public Private Partnership (5G PPP) Key Performance Indicators (KPIs)". [En línea]. Último acceso: Noviembre 2018. https: //5g-ppp.eu/kpis

[Adhik15] V. K. Adhikari, Y. Guo, F. Hao, V. Hilt, Z. Zhang, et. al., "Measurement Study of Netflix, Hulu, and a Tale of Three CDN", IEEE Transactions on Networking, vol. 23, no. 6, Diciembre 2016, pp. 1984-1997.

[Amaya13] N. Amaya, M. Irfan, G. Zervas, R. Nejabati, D. Simeonidou, et. al., "Fullyelastic multi-granular network with space/frequency/time switching using multi-core fibres and programmable optical nodes", Optics Express, vol. 21, no. 7, Abril 2013, pp. $8865-8872$.

[Aws] Amazon, “AWS, Amazon Web Service”. [En línea]. Último acceso: Noviembre 2018. https: / / aws . amazon. com

[Arik13] S.O. Arik, J. M. Kahn, "Coupled-Core Multi-Core Fibers for Spatial Multiplexing”, IEEE Photonics Technology Letters, vol. 25, no. 21, Noviembre 2013, pp. 2054-2057.

[Bang16] Y. Bang, J-K. K. Ree, K. Park, K. Lim, G. Nam, et. al., "CDN interconnection service trial: implementation and analysis", IEEE Communincations Magazine, vol. 54, no. 6, Junio 2016, pp. 94-100.

[Beaco] "Beacon". [En línea]. Último acceso: Noviembre 2018. https: / / openflow.stanford.edu/display/Beacon.

[Bjork10] M. Bjorklund, "YANG - A data modeling language for the network configuration protocol (NETCONF)", IETF RFC 6020, 2010.

[Borst10] S. Borst, V. Gupta and A. Walid, "Distributed caching algorithms for content distribution networks", en Proc. de International Conference on Computer Communications (INFOCOM), Marzo 2010.

[Cisco16a] "Cisco Global Cloud Index: Forecast and Methodology, 2015-2020 White Paper". 2016. 
[Cisco16b] "The Cisco Digital Network Architecture Vision - An Overview". White Paper. 2016.

[Cisco17] "Cisco Visual Networking Index: Forecast and Methodology, 2016-2021". 2017.

[Cisco18] "Cisco APIC Enterprise Module". 2018. Último acceso: Noviembre 2018. [En línea]. https://developer.cisco.com/site/apic-em

[Colli13] B. Collings, "New devices enabling software-defined optical networks", IEEE Communincations Magazine, vol. 51, no. 3, Marzo 2013, pp. 66-71.

[Cord] "Central Office Re-architected as a Datacenter (CORD) project". [En línea]. Último acceso: Noviembre 2018. https : / / opencord. org

[Elast] "Elastic Networks". [En línea]. Último acceso: Noviembre 2018. https://elasticnetworks.org

[Enns11] R. Enns, M. Bjorklund, J. Schoenwaelder, A. Bierman, "Network configuration protocol (NETCONF)", IETF RFC 6241, Junio 2011.

[Etsi14] "ETSI GS NFV 003 V1.2.1: Network Functions Virtualisation (NFV). Terminology for main concepts in NFV”, TSI Ind. Spec. Group (ISG), Diciembre 2017.

[Ferdo15] S. Ferdousi, F. Dikbiyik, M.F. Habib, M. Tornatore, B. Mukherjee, "DisasterAware Datacenter Placement and Dynamic Content Management in Cloud Networks", IEEE/OSA Journal of Optical Communications and Networking, vol. 7, no. 7, Julio 2017, pp. 681-694.

[Filer14] M. M. Filer, S. Tibuleac, "N-degree ROADM architecture comparison: broadcast-and-select versus route-and-select in $120 \mathrm{~Gb} / \mathrm{s}$ DP-QPSK transmission systems", en Proc. de Optical Fiber Conference (OFC), Marzo 2014.

[Flood18] "Project Floodlight", [En línea]. Último acceso: Noviembre 2018. http: / / www.projectfloodlight.org

[Garri15] M. Garrich, E. Magalhães, H. Carvalho, N.G. Gonzalez, G.S. Zervas, et. al., "Experimental Demonstration of Function Programmable Add/Drop Architecture for ROADMs", IEEE/OSA Journal of Optical Communications and Networking, vol. 7, no. 2, Febrero 2015, pp. 335-343.

[Garri18a] M. Garrich, F.J. Moreno Muro, M.V. Bueno Delgado, P. Pavon Mariño, "Open-source Network Optimization Software in the Open SDN/NFV Transport Ecosystem", IEEE/OSA Journal of Lightwave Technolgy, Septiembre 2018, DOI: 10.1109/JLT.2018.2869242

[Garri18b] M. Garrich, J.L. Romero-Gázquez, F.J. Moreno Muro, M.V. Bueno Delgado, P. Pavon Mariño, "The Latency Role in Dimensioning SDN/NFV-enabled Metro Networks", En revisión en IEEE/OSA Journal of Optical Communication and Networking, 2018. 
[Garri19a] M. Garrich, C. San-Nicolás-Martínez, F.J. Moreno-Muro, A. Mayoral Lopez-de-Lerma, O. Gonzalez de Dios, et. al., "Gap Analysis on Open Models for Partially-Disaggregated SDN Optical Transport Environments", En revisión en Optical Fiber Conference (OFC), 2019.

[Garri19b] M. Garrich, M. Hernández-Bastida, C. San-Nicolás-Martínez, F. J. MorenoMuro, P. Pavon-Marino, “The Net2Plan-OpenStack Project: IT Resource Manager for Metropolitan SDN/NFV Ecosystems", En revisión en en Optical Fiber Conference (OFC), 2019.

[Gerst12] O. Gerstel, M. Jinno, A. Lord, S. J. B. Yoo, "Elastic optical networking: a new dawn for the optical layer?", IEEE Communications Magazine, vol. 50, no. 2, Febrero 2012, pp. 12-20.

[Gerst15] O. Gerstel, "Control Architectures for Multi-Layer Networking: Distributed, centralized, or something in between?", en Proc. de Optical Fiber Conference (OFC), Marzo 2015.

[Goiri11] I. Goiri, K. Le, J. Guitart, J. Torres, and R. Bianchini, "Intelligent placement of datacenters for Internet services", en Proc. de International Conference on Distributed Computing Systems (ICDCS), Junio 2011, pp. 131-142.

[Googl] Google, “Google Cloud CDN”. [En línea]. Último acceso: Noviembre 2018. https://cloud.google.com

[Han15] B. Han, V. Gopalakrishnan, L. Ji, S. Lee, "Network Function Virtualization: Challenges and Opportunities for Innovations", IEEE Communications Magazine, vol. 53, no. 2, Feb. 2015, pp. 90-97.

[Ibm] IBM, “CPLEX Optimizer”. [En línea]. Último acceso: Noviembre 2018. https://ibm.com/analytics/cplex-optimizer

[Ietf06] Internet Engineering Task Force, "NETCONF Configuration Protocol. RFC 4741", 2006.

[Imran18] M. Imran, P. M. Anandarajah, A. Kaszubowska-Anandarajah, N. Sambo, L. Potí, "A Survey of Optical Carrier Generation Techniques for Terabit Capacity Elastic Optical Networks", IEEE Communications Surverys and Tutorials, vol.20, no. 1, Febrero 2018, pp. 211-263.

[ItuT12] International Telecommunication Union (ITU-T), "Spectral grids for WDM applications: DWDM frequency grid”, Ser. G.694.1, 2012.

[ItuT14] International Telecommunication Union (ITU-T), “The Tactile Internet, ITU-T Technology Watch Report", 2014.

[Jia17] Q. Jia, R. Xie, T. Huang, J. Liu, Y. Liu, “The Collaboration for Content Delivery and Network Infrastructures: A Survey”, IEEE Access, vol. 5, Junio 2017, pp. 1808818106. 
[Jom] "JOM - The Java Optimizer Modeler", [En línea]. Último acceso: Noviembre 2018. http://net2plan.com/jom.

[Khoda16] P.S. Khodasenas, J.M. Rivas-Moscoso, D. Siracusa, F. Pederzolli, “Comparison of spectral and spatial super-channel allocation schemes for SDM networks", IEEE/OSA Journal of Lightwave Technolgy, vol. 34, no. 11, Junio 2016, pp. 27102716.

[Kreut15] D. Kreutz, F. M. V. Ramos, P. E. Veríssimo, C. E. Rothenberg, S. Azodolmolky and S. Uhlig, "Software-Defined Networking: A Comprehensive Survey", en Proc. de IEEE, vol. 103, no. 1, Enero 2015, pp. 14-76.

[Linux] "The Linux Foundation", [En línea]. Último acceso: Noviembre 2018. https: //www. linuxfoundation.org.

[Lopez16] V. Lopez, L. Velasco, "Elastic Optical Networks. Architectures, Technologies and Control", Springer, 2016.

[Lument17] Lumentum, "New ROADM whitebox preliminary datasheet", 2017.

[Manso14] Y. Mansouri, S. Azad and A. Chamkori, "Minimizing cost of K-replica in hierarchical data grid environment", en Proc. de International Conference on Advanced Information Networking and Application (AINA), Mayo 2014, pp. 1073-1080.

[Marom15] D. M. Marom, M. Blau, "Switching solutions for WDM-SDM optical networks", IEEE Communications Magazine, vol. 53, no. 2, Febrero 2015, pp. 60-68.

[Marom17] D. M. Marom, P. D. Colbourne, A. D'Errico, N. K. Fontaine, Y. Ikuma, et. al., "Survey of photonic switching architectures and technologies in support of spatially and spectrally flexible optical networking", IEEE/OSA Journal of Optical Communications and Networking, vol. 9, no. 1, Enero 2017, pp. 1-26.

[Metro] "Metro-Haul Project", [En línea]. Último acceso: Noviembre 2018. https://metro-haul.eu.

[Micros18] “Microsoft Azure, ¿qué es la nube?”, [En línea]. Último acceso: Noviembre 2018. https://azure.microsoft.com.

[Mijum16] R. Mijumbi, J. Serrat, J. L. Gorricho, et. al., "Network Function Virtualization: State-of-the-Art and Research Challenges", IEEE Communications Surverys and Tutorials, vol.18, no. 1, Mar. 2016, pp. 236-262.

[Moren17] F.J. Moreno Muro, R. Rumipamba-Zambrano, P. Pavon-Marino, J. Perello, J.M. Gene, S. Spadaro, "A Evaluation of Core-Continuity-Constrained ROADMs for Flex-Grid/MCF Optical Networks", IEEE/OSA Journal of Optical Communications and Networking, vol. 11, no. 11, Noviembre 2017, pp. 1041-1050.

[Moren18a] F.J. Moreno Muro, N. Skorin-Kapov, P. Pavón Mariño, "Revisiting Core Traffic Growth in the Presence of Expanding CDNs", En revisión en Elservier Computer Networks, 2018. 
[Moren18b] F.J. Moreno Muro, C. San Nicolás Martínez, E. Martín Seoane, M. Garrich, P. Pavon Mariño, et. al., "Joint Optimal Service Chain Allocation, VNF instantiation and Metro Network Resource Management Demonstration", en Proc. de Optical Fiber Conference (OFC), Marzo 2018.

[Moren18c] F.J. Moreno Muro, R. Rumipamba-Zambrano, J. Perelló, et. al., "Elastic Networks thematic network results I: planning and control of Flex-Grid/SDM", en Proc. de International Conference on Transparent Optical Networks (ICTON), Julio 2018.

[Moren18d] F.J. Moreno Muro, C. San Nicolás Martínez, M. Garrich, P. Pavon Mariño, O. Gónzalez de Dios, et. al, "Latency-aware Optimization of Service Chain Allocation with joint VNF instantiation and SDN metro network control", en Proc. de European Conference on Optical Communication (ECOC), Septiembre 2018.

[Muham15] A. Muhammad, G. Zervas, R. Forchheimer, "Resource allocation for space-division multiplexing: optical white box versus optical black box networking", IEEE/OSA Journal of Lightwave Technolgy, vol. 33, no. 23, Diciembre 2015, pp. 4928-4941.

[Muham17] A. Muhammad, N. Skorin-Kapov and M. Furdek, "Manycast, Anycast and Replica Placement (MARP) in Optical Inter-Datacenter Networks", IEEE/OSA Journal of Optical Communications and Networking, vol. 9, no. 12, Diciembre 2017, pp. 1161-1171.

[Napol15] A. Napoli, M. Bohn, D. Rafique, et. al., "Next generation elastic optical networks: The vision of the European research project IDEALIST", IEEE Comunications Magazine, vol. 53, no. 2, Febrero 2015, pp. 152-162.

[Nelso14] L. E. Nelson, M. D. Feuer, K. Abedin, X. Zhou, T. F. Taunay, "Spatial superchannel routing in a two-span ROADM system for space division multiplexing", IEEE/OSA Journal of Lightwave Technolgy, vol. 32, no. 4, Febrero 2014, pp. 783-789.

[Net2P] "Net2Plan - The Open Source Network Planner. ", [En línea]. Último acceso: Noviembre 2018. http: / / www . net2plan. com.

[Nox] N. Gude, T. Koponen, J. Pettit, B. Pfaff, M. Casado, et. al., "NOX: towards an operating system for networks", ACM SIGCOMM Computer Communication Review, vol. 38, no. 3, Julio 2008, pp. 105-110.

[Onap] "Open Network Automation Platform (ONAP)", [En línea]. Último acceso: Noviembre 2018. wWw . onap . org.

[Onos] "Open Networking Operating System (ONOS)", [En línea]. Último acceso: Noviembre 2018. onosproject. org.

[Onofr] "Project ONOFRE, Optical Networks Convergence in the Future Internet", [En línea]. Último acceso: Noviembre 2018. http://girtel.upct.es/projects/onofre. 
[Onof2] "Project ONOFRE-2, Optical Networks Convergence in the Future Internet - 2", [En línea]. Último acceso: Noviembre 2018. http://girtel. upct.es/projects/onofre2.

[OpenC] "OpenConfig", [En línea]. Último acceso: Noviembre 2018. http://www. openconfig.net.

[OpenD] "Open Daylight", [En línea]. Último acceso: Noviembre 2018. https: / / www.opendaylight.org.

[OpenN12] "Open Networking Foundation. Software-Defined Networking: The New Norm for Networks", [En línea], White Paper, Abril 2012.

[OpenN13] "Open Networking Foundation. OpenFlow Swithch Specification.”, [En línea], Octubre 2013. Último acceso: Noviembre 2018.

[OpenN17] “Open Networking Foundation. Transport API (TAPI) 2.0 Overview”, [En línea], White Paper, Agosto 2017. Último acceso: Noviembre 2018.

[OpenN18] “The Open Networking Foundation", [En línea]. Último acceso: Noviembre 2018. https: / / www. opennetworking.org/.

[OpenR] "OpenROADM", [En línea]. Último acceso: Noviembre 2018. http: / / www. openroadm.org.

[OpenS] "OpenStack", [En línea]. Último acceso: Noviembre 2018. https: //www. openstack.org.

[Osm] "ETSI Open Source MANO”, [En línea]. Último acceso: Noviembre 2018. https://osm.etsi.org.

[Pavon15] P. Pavon-Marino, J.-L. Izquierdo-Zaragoza, "Net2Plan: An open source optical network planning tool for bridging the gap between academia and industry", IEEE Network Magazine, vol. 29, no. 5, Octubre 2015, pp. 90-96.

[Pavon16] P. Pavon-Marino, "Optimization of Computer Networks. Modeling and Algorithms: A Hand-On Approach”, Wiley, 2016.

[Pavon17] P. Pavon-Mariño, F.J. Moreno Muro, N. Skorin-Kapov, "Evolution of Core Traffic for Growing CDNs: Is the growth rate of core network traffic overestimated?", en Proc. de Optical Fiber Conference (OFC), Marzo 2017.

[Pavon18] P. Pavon-Mariño, M. Garrich, F.J. Moreno Muro, "The Role of Open-source Network Optimization Software in the SDN/NFV World", en Proc. de Optical Fiber Conference (OFC), Marzo 2018.

[Perel16a] J. Perello, K. Walkowiak, M. Klinkowski, S. Spadaro, D. Careglio, "Joint content placement and lightpath routing and spectrum assignment in CDNs over elastic optical network scenarios", Computer Communications, vol. 77, Marzo 2016, pp. 7284. 
[Perel16b] J. Perelló, J. M. Gené, A. Pagès, J. A. Lazaro, S. Spadaro, "Flex-grid/SDM backbone network design with inter-core XT limited transmission reach", IEEE/OSA Journal of Optical Communications and Networking, vol. 8, no. 8, Agosto 2016, pp. 540-552. Poggiolini

[Poggi14] P. Poggiolini, G. Bosco, A. Carena, V. Curri, Y. Jiang, et. al., "The GN-Model of Fiber Non-Linear Propagation and its Applications", Journal of Lightwave Technology, vol. 32, no. 4, Febrero 2014, pp. 694-721.

[Pox] "POX Platform", [En línea]. Último acceso: Noviembre 2018. https://github.com/noxrepo/pox.

[Puttn17] B. J. Puttnam, R. S. Luis, E. Agrell, G. Rademacher, J. Sakaguchi, et. al., "High capacity transmission systems using homogeneous multi-core fibers", Journal of Lightwave Technology, vol. 35, no. 6, Marzo 2016, pp. 1157-1167.

[Ricca18] E. Riccardi, P. Gunning, O. Gonzalez de Dios, M. Quagliotti, V. Lopez, et . al., "An Operator's view on introduction of White Boxes in Optical Networks", Journal of Lightwave Technology, vol. 36, no. 15, Agosto 2018, pp. 3062-3072.

[Rivas17] J. M. Rivas-Moscoso, B. Shariati, D. M. Marom, D. Klonidis, I. Tomkos, et. al., "Comparison of $\mathrm{CD}(\mathrm{C}) \mathrm{ROADM}$ Architectures for Space Division Multiplexed Networks", en Proc. de Optical Fiber Conference (OFC), Marzo 2017.

[Romer18] J. L. Romero-Gázquez, M. V. Bueno-Delgado, F. J. Moreno-Muro, P. PavónMarino, "Net2plan-GIS: An open-source Net2Plan extension integrating GIS data for 5G network planning", en Proc. de International Conference on Transparent Optical Networks (ICTON), Junio 2018.

[Ruiz14] M. Ruiz, L. Velasco, A. Lord, D. Fonseca, M. Pióro,, et. al., "Planning fixed to flexgrid gradual migration: Drivers and open issues", IEEE Comunications Magazine, vol. 52, no. 1, Enero 2014, pp. 70-76.

[Rumip17a] R. Rumipamba-Zambrano, F.J. Moreno Muro, P. Pavón-Marino, J. Perelló, S. Spadaro, et. al., "Assessment of Flex-Grid/MCF Optical Networks with ROADM Limited Core Switching Capability", en Proc. de Optical Network Design and Modelling (ONDM), Junio 2017.

[Rumip17b] R. Rumipamba-Zambrano, J. Perelló, J. M. Gené, S. Spadaro, “Capacity Quantification of Joint-switching-enabled Flex-Grid/SDM Optical Backbone Networks", en Proc. de Optical Fiber Conference (OFC), Marzo 2017.

[Rumip18] R. Rumipamba-Zambrano, F.J. Moreno Muro, J. Perelló, P. Pavón-Marino, S. Spadaro, "Space Continuity Constraint in Dynamic Flex-Grid/SDM Optical Core Networks: An Evaluation with Spatial and Spectral Super-channels", Computer Communications), vol. 126, Agosto 2018, pp. 38-49.

[Sahoo17] J. Sahoo, M.A. Salahauddin, R. Glitho, H. Elbiaze, W. Ajib, “A Survey on Replica Placement Algorithms for Content Delivery Networks", IEEE Communications Surveys and Tutorials, vol. 19, no.2, Noviembre 2017, pp. 1002-1026. 
[Sakag12] J. Sakaguchi, Y. Awaji, N. Wada, A. Kanno, T. Kawanishi, et. al., "Space division multiplexed transmission of 109-Tb/s data signals using homogeneous sevencore fiber", Journal on Lightwave Technology, vol. 30, no. 4, Febrero 2012, pp. 658665 .

[Sakag14] J. Sakaguchi, W. Klaus, B. J. Puttnam, J. M. D. Mendinueta, Y. Awaji, et. al., "19-core MCF transmission system using EDFA with shared core pumping coupled via free-space optics", Optics Express, vol. 22, no. 1, Enero 2014, pp. 90-95.

[Salah17] M. A. Salahuddin, J. Sahoo, R. Glitho, H. Elbiaze, W. Ajib, "Survey on Content Placement Algorithms for Cloud-based Content Delivery Networks", IEEE Access), vol. 6, Septiembre 2017, pp. 91-114.

[Salma16] O. Salman, I. H. Elhajj, A. Kayssi, A. Chehab, "SDN Controllers: A Comparative Study", en Proc. de Mediterranean Electrotechnical Conference (MELECON), Abril 2016.

[Sandv16] "Sandvine 2016, Global Internet Phenomena", 2016.

[Sandv18] "Sandvine 2018, Global Internet Phenomena", 2018.

[Sdnli17] "SDNlib", [En línea], 2017. Último acceso: Noviembre 2018. sndlib.zib.de

[Shali13] A. Shalimov, D. Zuikov, D. Zimarina, V. Pashkov, R. Smeliansky, "Advanced Study of SDN/OpenFlow controllers", en Proc. de Central and Eastern European Software Engineering Conference CEE-SECR, Oct. 2013.

[Svalu13] M. Svaluto, J.M. Fàbrega, L. Nadal, F.J. Vílchez, G. Junyent, "Bandwidth variable transponders based on OFDM technology for elastic optical networks", en Proc. de International Conference on Transparent Optical Networks (ICTON), Junio 2013.

[Tanak13] T. Tanaka, A. Hirano, M. Jinno, "Impact of Transponder Architecture on the Scalability of Optical Nodes in Elastic Optical Networks", IEEE Communications Letters), vol. 17, no. 9, Sept. 2013, pp. 1846-1848.

[Wizne15] P.J. Wizner, "Spatial multiplexing: The next frontier in network capacity scaling”, en Proc. de European Conference on Optical Communication (ECOC), Sept. 2013.

[Wu15] Y. Wu, M. Tornatore, S. Thota, B. Mukherjee, "Renewable-Energy-Aware Data Center Placement in Optical Cloud Network", en Proc. de Optical Fiber Conference (OFC), Mar. 2015.

[Yilma18] O.F. Yilmaz, S. St-Laurent, M. Mitchell "Automated Management and Control of a Multi-Vendor Disaggregated Network at the L0 Layer", en Proc. de Optical Fiber Conference (OFC), Mar. 2018. 
[Yin17] H. Yin, X. Zhang, S. Zhao, Y. Luo, C. Tian, V. Sekar, "Tradeoffs Between Cost and Performance for CDN Provisioning Based on Coordinate Transformations", IEEE Transactions on Multimedia, vol. 11, no. 19, Nov. 2017, pp. 2583-2596.

[Zhu16] M. Zhu, D. Li, F. Wang, A. Li, K.K. Ramakrishnan, et. al., ““'CCDN: ContentCentric Data Center Networks", IEEE/ACM Transactions on Networking, vol. 24, no. 6, Dec. 2017, pp. 3537-3550. 



\section{Acrónimos y abreviaciones}

5G $5^{\text {a }}$ Generación de redes móviles

AoD Architecture on Demand

API Application Programming Interface

BPSK Binary Phase-Shift Keying

BVT Bandwidth Variable Transponder

CAGR Compound Annual Growth Rate

CAPEX CAPital EXpenditures

CDN Content Delivery Network

CPU Central Processing Unit

CU Content Unit

DC Data Center

DSP Digital Signal Processing

eMBB enhanced Mobile BroadBand

EPA Enhanced Platform Awareness

FMF Few-Mode Fiber

FM-MCF Few-Mode Multi-Core Fiber

FNB-ROADM Fully Non-Blocking ROADM

FPI Formación de Personal Investigador

GB Guard Band

Gbps Giga Bits Per Second 
GUI Graphical User Interface

HD Hard Disk

HDD Hard Disk Device

IaaS Infraestructure as a Service

ICXT Inter-Core CrossTalk

ILP Integer Linear Program

IMT International Mobile Telecomunications

InS Independent-Switching

IoT Internet of Things

IP Internet Protocol

IT Information Technology

ITU International Telecommunications Union

JoS Joint-Switching

JOM Java Optimizer Modeller

KPI Key Performance Indicator

LA-SCCE Latency-Aware Service Chain Computation Element

MAC Medium Access Control

MARP Manycast, Anycast and Replica Placement

MEC Multi Edge Computing

MIMO Multiple-Input Multiple-Output

MMF Multi-Mode Fiber

mMTC massive Machine Type Communications

NaaS Networking as a Service

NAT Network Address Translation

NBI North Bound Interface

NETCONF NETwork CONFiguration protocol

NFV Network Function Virtualization 
NFVI Network Function Virtualization Infrastructure

NFV MANO Network Function Virtualization MANagement and Orchestration

OADM Optical Add/Drop Multiplexer

ONOFRE Optical Networks Convergence in The Future Internet

OPEX OPeration EXpenditures

OaaS Optimization as a Service

P2P Peer to Peer

PCE Path Computation Element

PPP Public-Private-Partnership

QAM Quadrature Amplitue Modulation

QoS Quality of Service

QoT Quality of Transmission

QPSK Quadrature Phase-Shift Keying

RAM Random Access Memory

RCSA Routing, Core and Spectrum Assignment

RMSCA Routing, Modulation, Spectrum and Core Assignment

RMSSA Routing, Modulation, Spectrum and Space Assignment

ROADM Reconfigurable Optical Add/Drop Multiplexer

RSA Routing and Spectrum Assignment

RWA Routing and Wavelength Assignment

SBI South Bound Interface

SbC Sub Channel

SCC Spatial-Continuity-Constraint

SCCE Service Chain Computation Element

SCh Super Channel

SDM Space Division Multiplexing

SDN Software-Defined Networking 
SMF Single Mode Fiber

SDN Software Defined Network

SSD Solid State Device

SSH Secure SHell

SSS Spectrum Selective Switch

TR Transmission Reach

VCA VNF Configuration and Abstraction

VM Virtual Machine

VIM Virtual Infrastructure Manager

VNF Virtual Network Function

VoD Video on Demand

URLLC Ultra Reliable Low Latency Communications

WAN Wide Area Network

WDM Wavelength Division Multiplexing

WSS Wavelength Selective Switch 
Este trabajo ha sido financiado por el "Programa Estatal de Promoción del Talento y su Empleabilidad en I+D+i" perteneciente al Subprograma Estatal de Formación dependiente del Ministerio de Ciencia y Educación, proyecto ONOFRE (TEC2014-53071-C3-1-P), por el programa el "Programa Estatal de investigación Científica y Técnica de Excelencia", Subprograma Estatal de Generación de Conocimiento, proyectos "Elastic Networks" (TEC2015-71932-REDT) y ONOFRE-2 (TEC2017-84423-C3-1-P), y el proyecto Europeo Metro-Haul, programa H2020 (H2020-ICT-2016-2 G.A. 761727) financiado por la Unión Europea. 
